\title{
Inertial Particle Separator Multiphase Dynamics
}

\author{
A Dissertation \\ Presented to \\ the faculty of the School of Engineering and Applied Science \\ University of Virginia \\ In partial fulfillment \\ of the requirements for the degree \\ Doctor of Philosophy \\ by
}

Dominic L. Barone

May 2014 
(C) 2014 Dominic L. Barone 


\section{APPROVAL SHEET}

The dissertation

is submitted in partial fulfillment of the requirements

for the degree of

Doctor of Philosophy

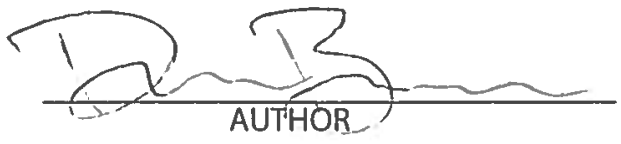

The dissertation has been read and approved by the examining committee:

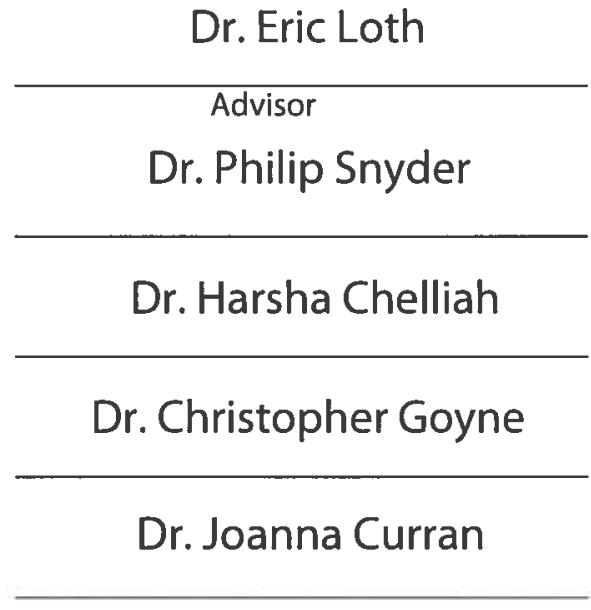

Accepted for the School of Engineering and Applied Science:

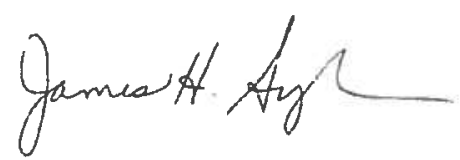

Dean, School of Engineering and Applied Science

May

2014 


\section{Abstract}

The effects of sand and dust ingestion often limit the useful life of turbine engines operating in austere environments and efforts are needed to reduce the quantity of particulate entering the engine. Several Engine Air Particle Separation (EAPS) systems exist. In particular, Inertial Particle Separators (IPS) are of interest because they offer significant weight savings and are more compact. However, they do not yet provide separation efficiencies as high as that from barrier filter and vortex-tube separator technologies. In order to further improve the efficiency of IPS systems, an in depth study of the multiphase flow dynamics has been undertaken.

An experimental approach was chosen to fill the void of available data and provide useful information for model validation. A wind tunnel has been designed, constructed and tested to study the multiphase flow dynamics of an inertial particle separator. The experimental facility includes a rectangular test-section ideal for optical access, and is capable of reproducing full-scale flow and particle conditions seen in separator systems. Separation efficiencies were measured for A4 Coarse Test Dust over a range of operating conditions for several geometries. Results show that the separation efficiency is dependent on the scavenge flow split and strongly dependent on the outer surface geometry. Further separation efficiency tests where conducted using nominally sized $10 \mu \mathrm{m}, 35 \mu \mathrm{m}$, and $120 \mu \mathrm{m}$ glass spheres to eliminate the complexities of dust size, shape, and density that are present in Arizona Test Dust. A model was then created to determine the separation efficiency for particles of any given size. 
Several flow visualization techniques were performed to determine the fluid and particle dynamics. Oil-streak flow visualization was utilized to characterize the flow along the walls of the IPS to determine the location of the recirculation zone and to verify that sidewall effects were minimal. Particle Image Velocimetry (PIV) was utilized to quantify the fluid flow field using small olive oil tracer particles. PIV was further utilized using $10 \mu \mathrm{m}$ and $35 \mu \mathrm{m}$ glass-spheres to determine the particle velocities in the IPS. Finally, high-speed video was used to capture the dynamics of the particle-fluid interactions. These experiments have shown the dynamic instabilities present in an IPS system that lead to lower separation efficiencies compared with other EAPS systems. The identification of these instabilities will help to improve future IPS designs. Also, the data collected during the course of this study provides the first reference of comparison for computational modeling of an IPS. 
For Rebecca. As one journey ends, another begins. 


\section{Acknowledgments}

Thank you to all of my family and friends, I would not have made it without your support. A special acknowledgement goes to my advisor, Dr. Eric Loth. He has not only put up with me

for the last few years, but he has taught me a great deal. I couldn't ask for a better advisor. To Phil Snyder from Rolls-Royce, our technical monitor, who provided me with insight and encouragement throughout the project. And, to all of the undergraduates who helped out in the lab and have some part in this document, Pete Miller, Peter Perley, Josh Coiner, Max Newman, and especially Jane Hawkins who's motivation and spirit were always refreshing. Thank to Rolls-Royce and the State of Virginia for providing funding for this project. 


\section{Contents}

Contents viii

List of Figures . . . . . . . . . . . . . . . . . . . . . . . . . . . . . . . . .

List of Tables . . . . . . . . . . . . . . . . . . . . xiv

$\begin{array}{lll}\text { Nomenclature } & \text { xvii }\end{array}$

1 Introduction 1

1.1 Document Organization . . . . . . . . . . . . . . . . . 1

1.2 Motivation . . . . . . . . . . . . . . . . . . 1

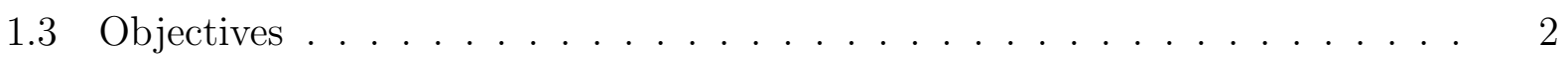

1.4 Hypothesis . . . . . . . . . . . . . . . . . . 3

2 IPS Research Facility $\quad 5$

2.1 Introduction . . . . . . . . . . . . . . . . . . 5

2.2 Facility and Experimental Methods . . . . . . . . . . . . . . . . . 9

2.3 Surface Flow Visualization Results . . . . . . . . . . . . . . . . . . . . . . 19

2.4 Separator Efficiency . . . . . . . . . . . . . . . . . . . . . . . . . . . . . . . . . . . 23

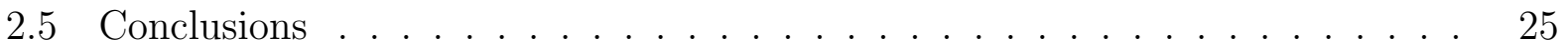

3 Efficiency of an Inertial Particle Separator $\quad 26$

3.1 Introduction . . . . . . . . . . . . . . . . . . . . . . . . . . . . . . .

3.2 Methodology . . . . . . . . . . . . . . . . . . 30

3.2.1 Flow Visualization . . . . . . . . . . . . . . . . . 32

3.2 .2 Efficiency Measurement . . . . . . . . . . . . . . . . . . . . 32

3.2 .3 Test Dust . . . . . . . . . . . . . . . . . . . . 34

3.3 Flow Visualization . . . . . . . . . . . . . . . . . . . 36

3.4 Efficiency Results . . . . . . . . . . . . . . . . . . . . . . . . 37

3.5 Conclusions . . . . . . . . . . . . . . . . . . . . . 38

4 Glass Sphere Efficiency $\quad 39$

4.1 Introduction . . . . . . . . . . . . . . . . . . . . . . . . . . . . . . . . . . . . . .

4.2 Methodology . . . . . . . . . . . . . . . . . . . . 42 
4.2.1 IPS Airflow and Geometry . . . . . . . . . . . . . . . . . 42

4.2.2 Particle Characteristics . . . . . . . . . . . . . . . . . . 45

4.2.3 Separation Efficiency Measurements . . . . . . . . . . . . . . 46

4.2 .4 Particle Stokes Number . . . . . . . . . . . . . . . . . 48

4.2.5 Separation Efficiency Model . . . . . . . . . . . . . . . . 50

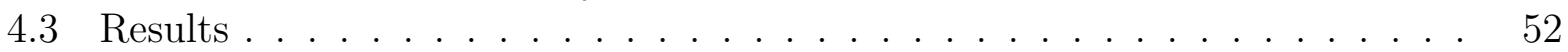

4.4 Conclusions . . . . . . . . . . . . . . . . . . . . . . 59

5 IPS Fluid Dynamics $\quad 60$

5.1 Introduction . . . . . . . . . . . . . . . . . . . . . 60

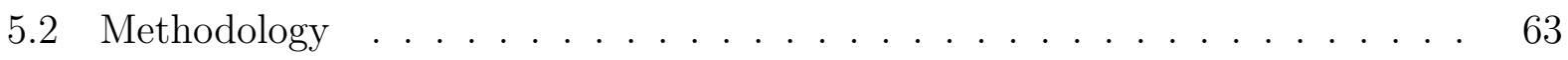

5.2 .1 Facility. . . . . . . . . . . . . . . . . . 63

5.2 .2 Particle Image Velocimetry of Gas Velocity . . . . . . . . . . . . . . . 65

5.2 .3 Data Convergence . . . . . . . . . . . . . . . . . 68

5.2.4 High-Speed Video of Glass Particle Dynamics . . . . . . . . . . . . . 69

5.3 Results . . . . . . . . . . . . . . . . . . 70

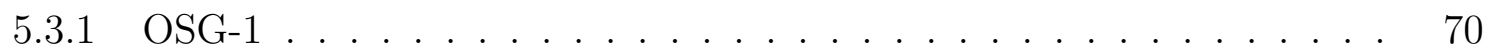

$5.3 .2 \quad \mathrm{OSG}-2 \ldots \ldots \ldots \ldots \ldots$

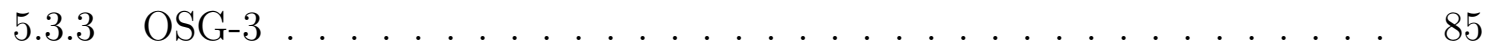

5.3.4 High Speed Video . . . . . . . . . . . . . . . . . . . . 90

5.4 Turbulent Length Scale Discussion . . . . . . . . . . . . . . . . . . . . 94

5.5 Conclusions . . . . . . . . . . . . . . . . . . . . . . . 94

6 IPS Particle Dynamics $\quad 96$

6.1 Methodology . . . . . . . . . . . . . . . . . . . . . . 99

6.1.1 Facility . . . . . . . . . . . . . . . . . . . . 99

6.1.2 Multiphase PIV Measurements . . . . . . . . . . . . . . . . 101

6.1.3 Particle Restitution . . . . . . . . . . . . . . . . . . . 102

6.2 Results ............................. . . . . 104

6.2 .1 OSG-1 . . . . . . . . . . . . . . . . . 104

6.2 .2 OSG-2 . . . . . . . . . . . . . . . . . . . . . . . . . . . . . . . . . . . . . . . . .

$6.2 .3 \quad \mathrm{OSG}-3 \ldots \ldots \ldots \ldots \ldots \ldots$

6.2.4 Particle Restitution . . . . . . . . . . . . . . . . . 115

6.3 Conclusions . . . . . . . . . . . . . . . . . . . . 120

7 Conclusions $\quad 121$

7.1 Objective 1: Facility Design . . . . . . . . . . . . . . . . . 121

7.2 Objective 2: Efficiency Measurements . . . . . . . . . . . . . . . . . . 122

7.3 Objective 3: Airflow and Particle Velocity Fields . . . . . . . . . . . . . . 123

7.4 Final Conclusions . . . . . . . . . . . . . . . . . . . . . . . . 124

7.5 Recommendations . . . . . . . . . . . . . . . . . . 125

$\begin{array}{lr}\text { References } & 127\end{array}$ 


\section{List of Figures}

2.1 Inertial particle separator geometry . . . . . . . . . . . . . . . 6

2.2 Expected air flow in an IPS . . . . . . . . . . . . . . . . 8

2.3 Photographs of UVa IPS Facility showing a) the IPS research tunnel and b) the air handling facility with the scavenge leg blower on the left and the core leg blower on the right . . . . . . . . . . . . . . . . . . 12

2.4 IPS research facility schematic showing the Particle Delivery System, air intake, IPS test section, diffusers, and particle filters. All dimensions are in inches .

2.5 Particle Delivery System, an accurate 106M screw feeder with attached aeration nozzle: a) PDS schematic b) PDS picture, and c) particle aeration operation

2.6 Influence of air pressure on PDS aeration; sand aeration was explored for varying nozzle exit pressures . . . . . . . . . . . . . . . . .

2.7 Air Intake with 1/2 inch diameter, 3 inches long, hex-cell flow straightener installed (Aspect ratio of 6$) \ldots \ldots \ldots$

2.8 Test section with OSG-1, with a) schematic of the test section geometry (see Table 1) and b) picture of the test section. The inflow cross section is $8.00 \mathrm{x}$ 1.63 inches and the total length is 12 inches. . . . . . . . . . . . . . .

2.9 Oil-streak visualization of the OSG-1 flow path with three different intake configurations. Each case contains the original image on top and an annotated image below. From left to right: OSG-1 side view, case a) Baseline operation with the flow straightener on and the PDS air running, case b) Operation without the PDS air, and case c) Operation without the flow straightener installed and without the PDS air. For each case, the core flow rate is 1.71

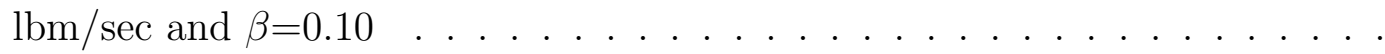

2.10 Oil-streak visualization of the OSG-1 flow path for scavenge split values of $\beta=0.10$ and $\beta=0.16$. There is little difference between the case of a) $\beta=0.10$ and b) $\beta=0.16$ in the location of the recirculation zone and the presence of the corner wall vortices. . . . . . . . . . . . . . . .

2.11 Oil-streak visualization of the core side of the hub for the OSG-1 flow path. Core flow rate of $1.71 \mathrm{lbm} / \mathrm{sec}$ and $\beta=0.10 \ldots \ldots$. . . . . . . . 22

3.1 Inertial particle separator geometry . . . . . . . . . . . . . . 27 
3.2 Qualitative Mach contours and streamlines to illustrate the recirculation zone present in the scavenge flow. . . . . . . . . . . . . . . . . . . 28

3.3 Previous IPS efficiency data relevant to current IPS research . . . . . . . . . 29

3.4 Facility schematic, dimensions are in inches . . . . . . . . . . . . . . 31

3.5 Test section geometries . . . . . . . . . . . . . . . . . . . . . . . . . . . . . . . . . . . . .

3.6 A4 Coarse Test Dust . . . . . . . . . . . . . . . . . . . . . 35

3.7 Test dust domain Stokes Number . . . . . . . . . . . . . . . . . 36

3.8 Oil streak images of each OSG at $\beta=0.16$ comparing recirculation zone size 37

3.9 IPS Efficiency for A4 Coarse Test Dust . . . . . . . . . . . . . . 38

4.1 IPS nomenclature and flow features . . . . . . . . . . . . . . . . . . 40

4.2 Previous studies of IPS Efficiency using A4 Coarse Test Dust ${ }^{[1-5]} \ldots . . . .41$

4.3 IPS test section geometries . . . . . . . . . . . . . . . . . . 41

4.4 IPS Research Facility located at the University of Virginia showing: 1) the particle delivery system, 2) air intake, 3) test section, 4) core filter, 5) scavenge filter, and 6) facility air system . . . . . . . . . . . . . . . .

4.5 Microscope images of glass-spheres for the three nominal diameters: a) 120 $\mu m$, b) $35 \mu m$ and c) $10 \mu m$ (note that images are at differing magnifications) 46

4.6 Microscope images of glass-spheres from scavenge filter . . . . . . . . . . . 47

4.7 Stokes numbers based on domain length (D) and throat height $(\mathrm{H})$ as a function of particle size . . . . . . . . . . . . . . . . . . . . . . . . . . . . . . . . . 49

4.8 Measured size distribution of glass-spheres . . . . . . . . . . . . . . . . . 50

4.9 Adaptation of the model from Langmuir and Blodgett ${ }^{[6]}$ to the IPS geometry 51

4.10 Glass bead efficiency as a function of flow split for different diameters a) 10

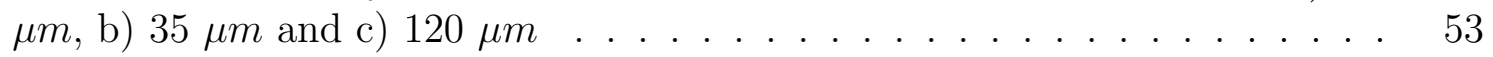

4.11 Glass bead efficiency as a function of diameter with a flow split of $\beta=0.16$. 55

4.12 Efficiency predictions based on previous A4 experiential data . . . . . . . . 55

4.13 Efficiency prediction comparison for $\beta=0.16$ showing a) OSG-1 $(C=0.046)$,

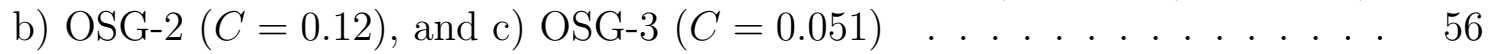

4.14 Efficiency prediction comparison $(C=0.04)$ for OSG-1 showing a) $\beta=0.14$, b) $\beta=0.16$, and c) $\beta=0.20 \ldots \ldots \ldots \ldots$

4.15 Efficiency prediction model applied to the data from Vittal et al ${ }^{[4]}$ and Hamed et al. ${ }^{[7]} \ldots \ldots \ldots \ldots \ldots \ldots$

5.1 Experimental setup showing a) schematic of the PIV setup and b) a picture of the high speed video setup . . . . . . . . . . . . . . . . 64 64

5.2 Olive-oil seed bar installed in the IPS research tunnel . . . . . . . . . . . . 67

5.3 Data Convergence, showing a) Statistical convergence of velocity and turbulence data at $x=19.2 \mathrm{~cm}$ and $y=6.6 \mathrm{~cm}$ and $\mathrm{b}$ ) number of vectors required to reach $5 \%$ convergence for the entire flow field. . . . . . . . . . . . . 69

5.4 Average quantities based on PIV measurements for OSG-1 at $\beta=0.16$. . 72 
5.5 Mass flux control volume showing a) the control volume with superimposed normal velocity vectors and the normal velocity component and \pm 1 standard deviation for b) the incoming profile, c) the core exit profile, and d) the scavenge exit profile. . . . . . . . . . . . . . . .

5.6 Local fluid volume flux scatter plot showing a) flux by component and b) the net flux. Note that flux is considered positive entering the control volume and negative exiting the volume. . . . . . . . . . . . . . . . .

5.7 Control volume flux for OSG-1 (bin size of $15 \mathrm{~m}^{2} / \mathrm{s}$ ) . . . . . . . . . . . . .

5.8 Instantaneous results for OSG-1 showing a) Mean control-volume flux, $\mu_{q}$, representative of mean IPS operation, b) Low control-volume flux, $\mu_{q}-2 \sigma_{q}$, caused by high-speed scavenge flow, and c) high control-volume flux, $\mu_{q}+2 \sigma_{q}$, indicative of ingested scavenge flow . . . . . . . . . . . . . .

5.9 Average quantities based on PIV measurements for OSG-2 at $\beta=0.16 \ldots 81$

5.10 Average normal velocity profiles for mass flux control volume . . . . . . . . . 82

5.11 Control volume flux for OSG-2 (bin size of $15 \mathrm{~m}^{2} / \mathrm{s}$ ) . . . . . . . . . . . 83

5.12 Instantaneous results for OSG-2 showing a) Mean control-volume flux, $\mu_{q}$, representative of mean IPS operation, b) Low control-volume flux, $\mu_{q}-2 \sigma_{q}$, caused by high-speed scavenge flow, and c) high control-volume flux, $\mu_{q}+2 \sigma_{q}$, indicative of ingested scavenge flow . . . . . . . . . . . . . . . 84

5.13 Average quantities based on PIV measurements for OSG-3 at $\beta=0.16$. . 86

5.14 Average normal velocity profiles for mass flux control volume . . . . . . . 87

5.15 Control volume flux for OSG-3 (bin size of $15 \mathrm{~m}^{2} / \mathrm{s}$ ) . . . . . . . . . . 88

5.16 Instantaneous results for OSG-3 showing a) Mean control-volume flux, $\mu_{q}$, representative of mean IPS operation, b) Low control-volume flux, $\mu_{q}-2 \sigma_{q}$, caused by high-speed scavenge flow, and c) high control-volume flux, $\mu_{q}+2 \sigma_{q}$, indicative of ingested scavenge flow . . . . . . . . . . . . . . . .

5.17 Embedded Video of OSG-1 with $10 \mu \mathrm{m}$ particles at $\beta=0.16$ showing a) and ingestion even and b) normal operation (click to play). Each video contains 180 frames at 20,000 frames per second, representing 9ms of elapsed time. Note that Adobe Reader 9 or higher with Adobe Flash Player is required to play the video. . . . . . . . . . . . . . . . . . . . . . . . . 91

5.18 High speed video results for OSG-1 showing ingestion event . . . . . . . . . . 92

5.19 High speed video results for OSG-1 showing expected operation . . . . . . . 93

6.1 Calculations from Hamed et al. ${ }^{[7]}$ that calculated using RANS as well as semiempirical drag model and probabilistic bounce models based on experimental data for a) $200 \mu m$, b) $10 \mu m$, and c) $3 \mu \mathrm{m}$ sand particles . . . . . . . . .

6.2 Experimental setup showing pictures of a) the wind tunnel showing the PDS (1), olive oil seed bar (2), tunnel air intake (3), test section (4), scavenge filter (5), core filter (6), and b) the PIV laser (7), and camera (8) . . . . . . 100

6.3 Diagram of particle bouncing . . . . . . . . . . . . . . . . 103

6.4 Restitution calculation location. . . . . . . . . . . . . . . . . 104

6.5 Average quantities of $10 \mu m$ particles for OSG-1 . . . . . . . . . 106 
6.6 Average quantities of $35 \mu m$ particles for OSG-1 . . . . . . . . . . . . . 108

6.7 Particle trace to streamline comparison for OSG-1 . . . . . . . . . . . . . . 109

6.8 Average quantities of $10 \mu m$ particles for OSG-2 . . . . . . . . . . . . . . 111

6.9 Average quantities of $35 \mu \mathrm{m}$ particles for OSG-2 . . . . . . . . . . . . . . 111

6.10 Particle trace to streamline comparison for OSG-2 . . . . . . . . . . . . . . . 112

6.11 Average quantities of $10 \mu m$ particles for OSG-3 . . . . . . . . . . . . 113

6.12 Average quantities of $35 \mu \mathrm{m}$ particles for OSG-3 . . . . . . . . . . . 113

6.13 Particle trace to streamline comparison for OSG-3 . . . . . . . . . . . . . . 114

6.14 Coefficient of Restitution for $10 \mu \mathrm{m}$ glass spheres as a function of location . . 115

6.15 Inbound and outbound wall normal velocity of $10 \mu \mathrm{m}$ particles. The mean velocities are filtered using a 5-point moving average to reduce noise in the $C O R_{\hat{n}}$ measurement. . . . . . . . . . . . . . . . 116

6.16 Schematic of three possible $10 \mu \mathrm{m}$ particle pathlines that match the small impact angles and high-speed video: a) Incoming high-speed particle path weakly affected by fluid forces, b) a particle bouncing multiple times across the OSG at shallow angles, and c) a slow particle that encounters an ingestion event. . . . . . . . . . . . . . . . . . . . 117

6.17 Coefficient of Restitution for $10 \mu \mathrm{m}$ glass spheres as a function of incoming perpendicular velocity . . . . . . . . . . . . . . . 117

6.18 Coefficient of Restitution for $35 \mu \mathrm{m}$ glass spheres as a function of location . . 118

6.19 Inbound and outbound wall normal velocity of $35 \mu \mathrm{m}$ particles. The mean velocities are filtered using a 5 -point moving average to reduce noise in the

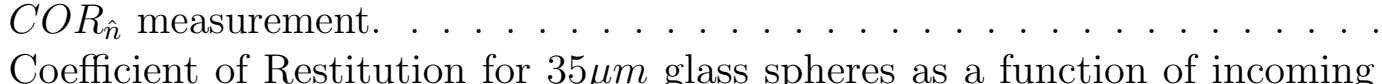
perpendicular velocity . . . . . . . . . . . . . . . 119 


\section{List of Tables}

4.1 Flow Characteristics ...................... 44

5.1 Summary of results from Barone et al. for efficiency and recirculation zone

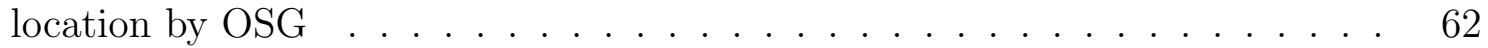

5.2 Test conditions for Gas PIV . . . . . . . . . . . . . . . . 64

5.3 PIV System . . . . . . . . . . . . . . . . 66

6.1 Description of particles used in MP-PIV measurements . . . . . . . . . . . 102 


\title{
Nomenclature
}

\author{
$c_{\forall} \quad$ added mass coefficient \\ $C_{D} \quad$ drag coefficient \\ $C O R$ coefficient of restitution \\ d diameter \\ $f \quad$ Stokes drag correction factor \\ $F_{D} \quad$ drag force \\ $\dot{m}$ mass flow rate \\ $m \quad$ mass \\ $\hat{n} \quad$ normal unit vector \\ $S t_{D}$ domain Stokes Number \\ $\hat{t} \quad$ tangent unit vector \\ $u \quad$ flow velocity \\ $\vec{w} \quad$ relative velocity vector \\ C constant
}




\section{Constants}

$c_{\forall} \quad$ added mass coefficient

$D \quad$ characteristic domain

d diameter

$H \quad$ throat height

$F \quad$ force

$f \quad$ Stokes drag correction factor

\section{Symbols}

$\beta \quad$ flow split fraction

$\Delta \quad$ difference

$\delta \quad$ uncertainty

$\eta \quad$ separation efficiency by mass

$\mu \quad$ dynamic viscoscity

$\rho \quad$ density

$\tau_{D} \quad$ domain response time

$p \quad$ particle

\section{Subscripts}

$b \quad$ balance

$c \quad$ core 


$\begin{array}{ll}c f & \text { core filter } \\ D & \text { characteristic domain } \\ n & \text { normal component } \\ P & \text { particle } \\ r & \text { residual } \\ s & \text { scavenge } \\ s f & \text { scavenge filter } \\ S t_{D} & \text { domain Stokes Number } \\ t & \text { tangential component } \\ t & \text { dynamic viscoscity } \\ t & \text { total } \\ & \\ & \end{array}$


xviii 


\section{CHAPTER 1}

\section{Introduction}

\section{$1.1 \quad$ Document Organization}

This dissertation is a summary of an experimental campaign conducted to elucidate the multiphase fluid dynamics present in an Inertial Particle Separator (IPS). This document is organized into seven chapters. The first chapter is an overview of the project's motivation and objectives. Chapters two through six are the five core chapters, each representing a different aspect of the research. Chapter seven is a summary of the conclusions and recommendations for further research. The core chapters are written as independent articles to consolidate pertinent information for the reader.

\subsection{Motivation}

An IPS is a device used to prevent dust and other debris from entering into the flow path of gas turbine engines. These devices are of particular use to vertical takeoff and landing aircraft operating in deserts where sand and dust are ubiquitous. IPS systems have reached 
a state where simple design tools are no longer capable of improving current design. In order to make further improvements in IPS separation efficiency, a fundamental understanding of the multiphase flow physics is required, particularly in relation to the airflow bifurcation characteristics. Understanding both the fluid flow field and the particulate motion in the splitter region is critical to achieve designs that maximize particle separation efficiency. The goal of this work is to discover the multiphase interactions occurring within the IPS bifurcation region and recirculation zone. Understanding how different particles react within an IPS is critical for improving design for future aircraft.

\subsection{Objectives}

The goal of this research was to find the underlying factors contributing to IPS efficiency in order to further advance IPS design. The project can be broken down into three objectives:

1. Develop a research facility capable of evaluating the research hypothesis

2. Use efficiency measurements for Arizona test dust and glass microspheres to evaluate particle size influence on efficiency

3. Explore multiphase interactions within the IPS using particle image velocimetry and particle tracking

The development of a research facility, was the first objective and the first necessary step in the experimental process. Objective 2 is set out to determine the baseline operation of the IPS geometries being studied. A rectangular IPS geometry has never been used before. Measurements using the Arizona test dust will serve as a baseline for comparison to other IPS geometry research. The use of glass microspheres will provide data for the effect of particle size on separation efficiency. Objective 3 ties the information together with direct tracking 
and analysis of the flow field and particles. Knowing the velocity field of the flow and of the particles is critical to understanding the interactions between them. Previous to this work, IPS research has been exclusively in the realm of computations.

\subsection{Hypothesis}

The hypothesis set out at the beginning of the project stated that the recirculation zone is unsteady, and this dynamic state is significant in particle separation efficiency. This premise was based on some possible mechanisms present in the flow field:

- Varying recirculation zone size

- Vortex shedding from recirculation zone

- Turbulence interactions within the recirculation zone

If the recirculation zone size changes with time, two particles of identical size and trajectory could take very different paths through the IPS, leading to a loss in efficiency. Vortex shedding from the recirculation zone could significantly affect particles entering the scavenge flow, and these types of flow structures are not accounted for in current IPS design.

Throughout the project, the hypothesis has evolved to incorporate the new information being discovered. Chapters 2, 3, and 4 confirm the dependence of separation efficiency to the recirculation zone size and location. In chapter 5, the dynamics of the recirculation zone are captured and the true mechanisms are unraveled. At this point the main hypothesis of an unsteady recirculation zone is expanding to include the entire scavenge flow leg, and three-dimensional effects are introduced. The three principles of the original hypothesis can be re-written to include this new information: 
- Unsteady scavenge flow

- Wall-normal vortex formation leading to core ingestion

- Turbulence interactions within bifurcation region

Chapter 6 then explores how small particles are impacted by the dynamic flow structures observed in chapter 5 . These results confirm the hypothesis and provide new insight into IPS particle separation. 


\section{CHAPTER 2}

\section{IPS Research Facility}

\section{$2.1 \quad$ Introduction}

When a turbine engine ingests sand or dust, the components of the engine may undergo highly damaging erosion, and the engine life can be significantly reduced ${ }^{[8-11]}$. This presents a major readiness, cost, and maintenance issue for military and civilian missions during low altitude operations in sandy areas such as the Middle East. ${ }^{[11]}$ Sand and dust separation devices traditionally fall into the three categories: Vortex Tube Separators (VTS), Inlet Barrier Filters (IBF), and Inertial Particle Separators (IPS). In contrast to VTS and IBF systems that are normally applied as airframe installed options, IPS systems are designed as integral to the engine. This integrated design results in a more compact system without blockage in the flow. For example, the inlet area needed for a VTS system is can be up to 10 times larger than the area required for an IPS system. ${ }^{[10]}$.

The basic features of an approximately axisymmetric IPS design ${ }^{[12]}$ are shown in figure 4.1. Particle-laden air enters the annular inlet and is bifurcated into two flow paths, each of which 


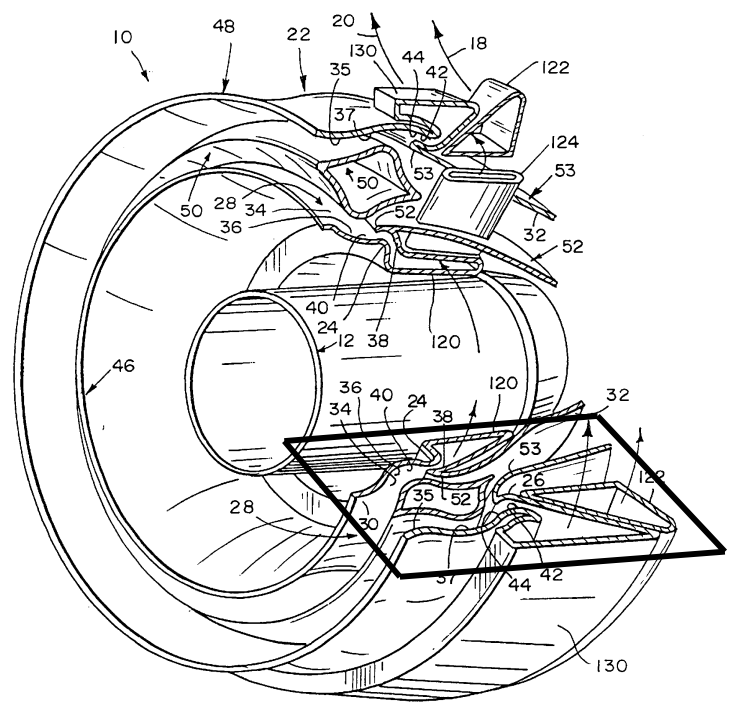

(a) IPS patent figure ${ }^{[12]}$

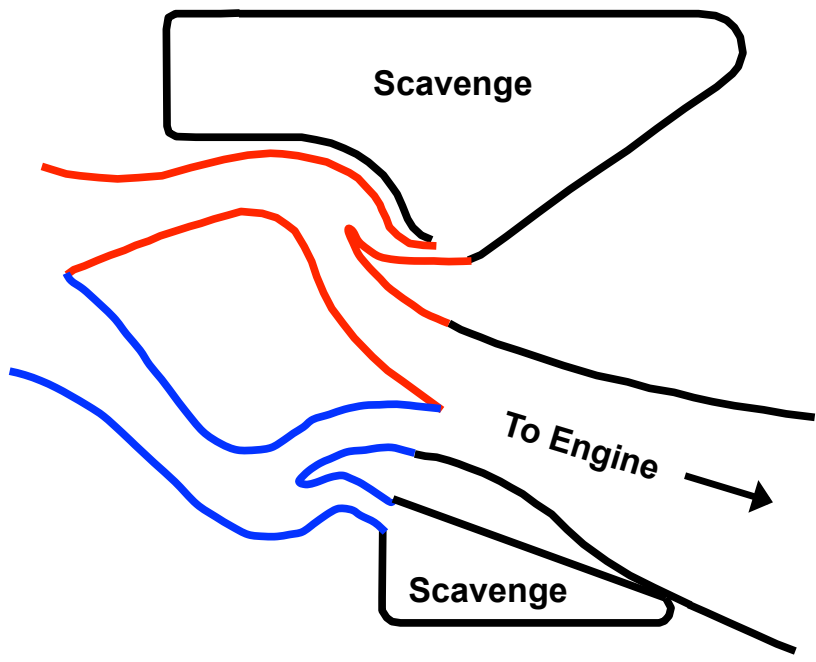

(b) Dual IPS flow path adapted from (a)

\section{Outer Surface Geometry (OSG)}

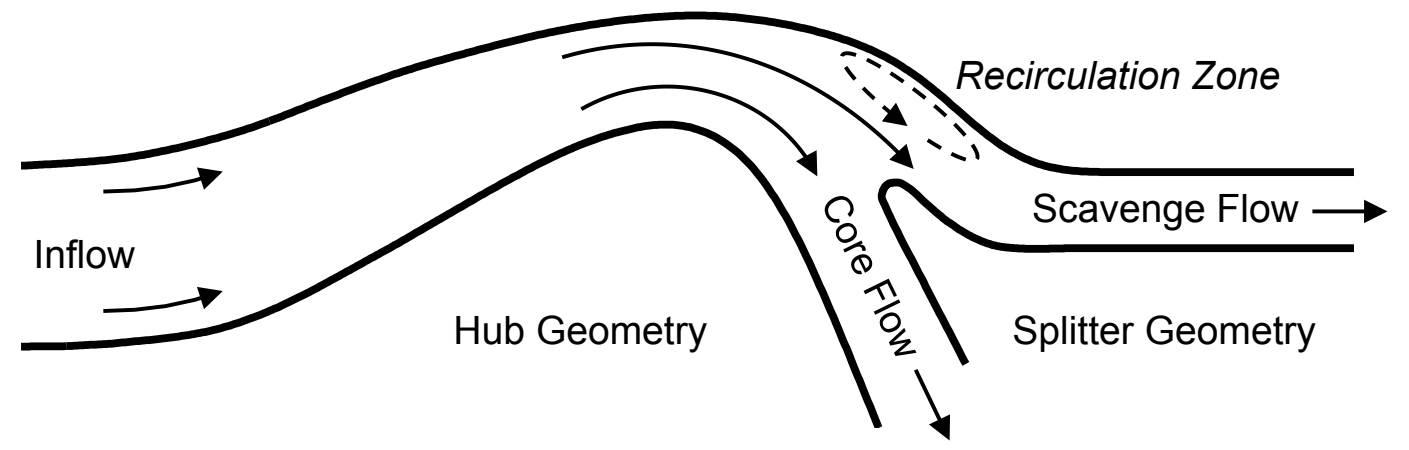

(c) Single path geometry and nomenclature

Figure 2.1: Inertial particle separator geometry

is then bifurcated by a set of splitters, with the main core flow rapidly turning while the scavenge flow continues in an approximately axial direction. In an installed IPS system, the mass flow of air to the core flow is driven by the turboshaft engine and the split to the scavenge flow is driven by some type of blower or ejector system.

A simplified schematic that identifies the outer set of core and scavenge paths (after the bifurcation) is shown in figure 4.1 Within the separator, the airflow makes a hub-side 
turn while the scavenge flow continues with very little flow turning. While very small dragdominated particles will tend to follow the streamlines, moderate size particles with sufficient inertia follow the scavenge path which prevents them from following the core airflow around this sharp corner. Large particles that are inertia-dominated will only be weakly effected by the flow and instead will be controlled more by their initial trajectories and any bouncing of solid surfaces. In general, the hub, splitter, and outer surface geometry are ideally designed to ensure that most of the particles enter the scavenge flow leg, so that the core flow is clean of damaging particulate. The scavenged particles are then ejected from the aircraft via the scavenge flow and the cleaned air proceeds to the turbine engine.

In general, IPS systems employ airflow scavenge splits (fraction of the mass flow diverted) of 0.10-0.30 and typically have separation efficiencies (fraction of the particles removed) between $50 \%$ and $85 \%$ for A4 coarse test dust. This is generally lower than that available for VTS and IBF systems, which have achieved efficiencies as high as $98 \%$ and $99 \%$, respectively ${ }^{[10]}$. As such, there is considerable room for improvement in IPS designs since increase in separation efficiency can lead to increased engine lifetime performance. For example, Prinsloo et al. ${ }^{[13]}$ noted that "If the separation efficiency of the inlet system increases from $94 \%$ to $95 \%$, the life expectancy [of the engine] is doubled, and if the efficiency then increases to $97 \%$, the life expectancy is doubled again." Ideally, IPS systems can be designed to have similar efficiencies of VTS and IBF systems, while maintaining their superiority in compactness and low pressure-loss performance.

In order to make these improvements in IPS separation efficiency, a fundamental understanding of the multiphase flow physics is required; particularly, in relation to the airflow bifurcation characteristics. Understanding of both the fluid flow field and the particulate motion in the splitter region is critical to achieve designs that maximize particle separation efficiency. A significant amount of effort has gone in to the computational studies ${ }^{[7,14-20]}$, 


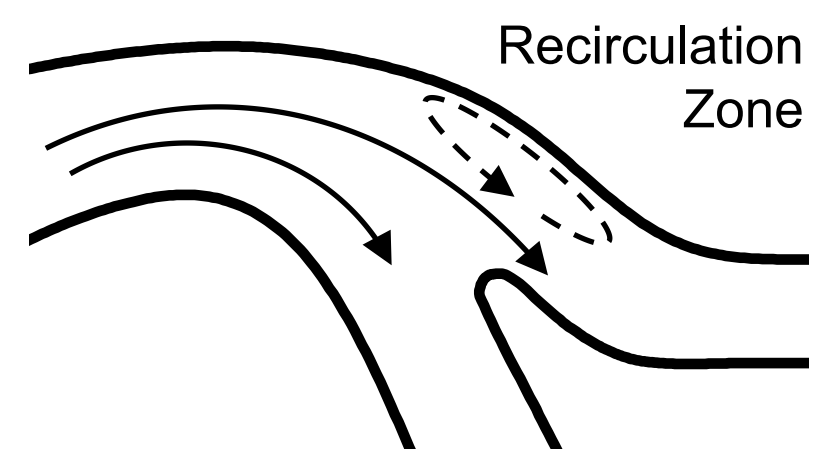

Figure 2.2: Expected air flow in an IPS

however these studies have focused primarily on the average flow field and do not account for dynamic flow structures.

Figure 2.2 illustrates the recirculation zone that exists along the outer surface of the flow path near the splitter. It is believed that this flow feature has a significant impact on separator efficiency although the details of the flow structure are poorly resolved by averaged flow solution techniques. The direct application of full three dimensional direct numerical flow simulation for the geometries is highly resource intensive and often out of scope for application to the problem. While computational simulations can allow quantitative results, the unsteady three-dimensional turbulent flow associated with an IPS geometry is very difficult to predict and computationally expensive. Similarly, the particle dynamics of an irregularly-shaped polydisperse mixture that includes significant wall reflections is difficult to model. Thus, prediction of separation efficiency and an understanding of the controlling flow phenomena influencing the particulate motion associated with the IPS are best obtained with an experimental approach and are needed to validate the computational models ${ }^{[20]}$.

To date, previous test facilities have only been designed to quantify the overall performance of IPS ${ }^{[1-4,21]}$; Duffy ${ }^{[2]}$ in particular did an extensive survey of three different geometries over a wide range of operating conditions. These studies have looked at the overall efficiency 
and aerodynamics of the particle separators with regard to installed performance, which can be used to verify the end result of a model, but provides little data to validate the model. Furthermore, previous facilities have employed axi-symmetric geometries that prevent use of advanced optical diagnostics such as Particle Image Velocimetery (PIV).

To the authors knowledge, no study has been done to explore the flow structures and multiphase interactions occurring within the separator, specifically, the dynamics present in the recirculation zone and its influence on particle trajectories. This is the first research facility created to explore the complex interactions present in the internal flow field of an IPS. This study describes a particle-laden wind tunnel facility with an optically accessible test section (via acrylic end walls) that can quantify the impact of particle characteristics, surface geometry and flow splits in terms of separation efficiency. This will be the first facility to quantify the internal flow field of an IPS and to track particles within the system. This facility will provide a complete geometry and data to validate the computational fluid and particle models for IPS systems and contribute knowledge critical to advance IPS designs.

\subsection{Facility and Experimental Methods}

A facility was designed to create the specific branching flow conditions present in an IPS system while allowing for the following measurements: overall sand separation efficiency, surface flow visualization, and quantitative optical internal flow diagnostics. Several facility requirements were identified in order to adequately explore the flow physics associated with the IPS system:

1. Flow Reynolds and Mach numbers consistent with actual IPS operation

2. Properly-scaled particle inertia and mass flow rate consistent with actual IPS operation 
3. Independently controlled scavenge and core flow legs to investigate effects of various flow rates and scavenge-core flow splits.

4. Method of measuring the overall IPS efficiency

5. Optical access to the test section for internal flow and particle velocity diagnostics

6. Test section which allows regions where end-wall effects are negligible and regions where end-wall effects are strong

7. Changeable outer surface geometry to explore different area changes and flow curvature

8. Physical access to the internal surfaces of the test section for surface flow visualization

Using full-scale conditions for the test section channel height and mass flows satisfies the first requirement. The second requirement is then simply satisfied by using a test dust with a consistent size, shape and density distribution. The third and fourth requirements led to a fan-pulled open-circuit wind tunnel geometry to ensure that the core and scavenge flow rates could be independently controlled. Moreover, this geometry prevents particle recirculation back into the inlet and facilitates a more uniform low-turbulence inflow. A direct connect facility also reduces design complexity and minimizes power requirements for tunnel operation. It has been shown that gravitational effects on particles in an IPS are negligible ${ }^{[20]}$, so the tunnel was oriented vertically to facilitate particle injection. To be consistent with typical IPS components for any particle-wall reflections, all flow-exposed surface were made of metal with the exception of windows for optical access. Metal surfaces also prevent build-up of static charges that can affect particle deposition.

The fifth requirement was important in choosing the test section geometry. Inertial particle separators are typically axi-symmetric flow paths (e.g. figure2.1) designed to be installed 
directly inline with turboshaft engines. Curved flow paths are notoriously difficult to examine with the optical measurement techniques planned in this study. A rectangular geometry also simplifies inlet and diffuser design, reducing the cost of the facility. Therefore, a rectangular 2-D IPS flow geometry was created, based on the equivalent area-change axis-symmetric flow geometry.

The sixth requirement was satisfied by use of a high-aspect ratio geometry with a large spanwise extent compared to the tunnel height. This should allow for a significantly twodimensional flow behavior near the centerline while also allowing a well-characterized end-wall flow region on the sides. This rectangular cross-section geometry also allowed the seventh and eighth requirements to be met by using replaceable outer surface geometries and separable inner surface geometry.

Stemming from the above design considerations, the resulting facility consists of five major sections, listed below in the order of the flow path: 1) particle delivery system, 2) air intake nozzle, 3) test section, 4) outlet diffusers, 5) particle filters, and 6) air handling system. A picture of the completed tunnel and a detailed drawing of the components are shown in figures 2.3 and 2.4 respectively. Details for each of the components are discussed in detail in the following text.

\section{Particle Delivery System}

In flow order, the first part of the tunnel is the particle delivery system (PDS). Located just above the intake the PDS is intended to simulate particle ingestion into the engine. The PDS is a commercially available auger, Accurate Model 106M, which is capable of variable feed rates to simulate different conditions using A4 Coarse Test Dust (ISO 12103-1) from Powder Technologies Inc. This dust is composed mostly of silica $\left(\mathrm{SiO}_{4}\right)$ and aluminum oxide $\left(\mathrm{Al}_{2} \mathrm{O}_{3}\right)$ but also contains several other components; it has a size distribution from 0 to 200 microns. 


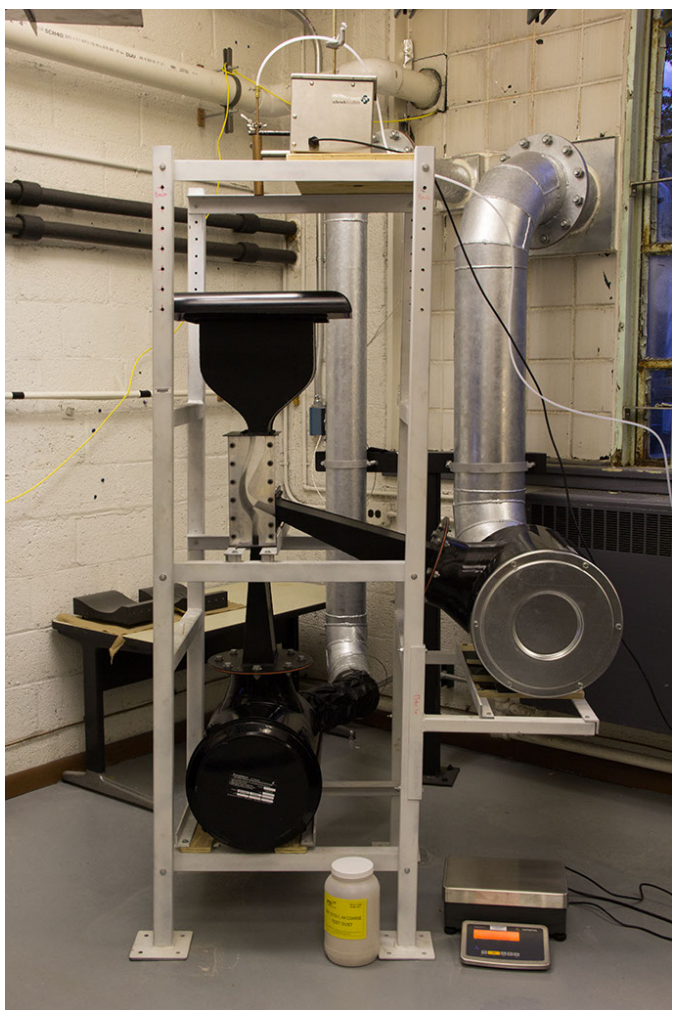

(a) IPS research tunnel

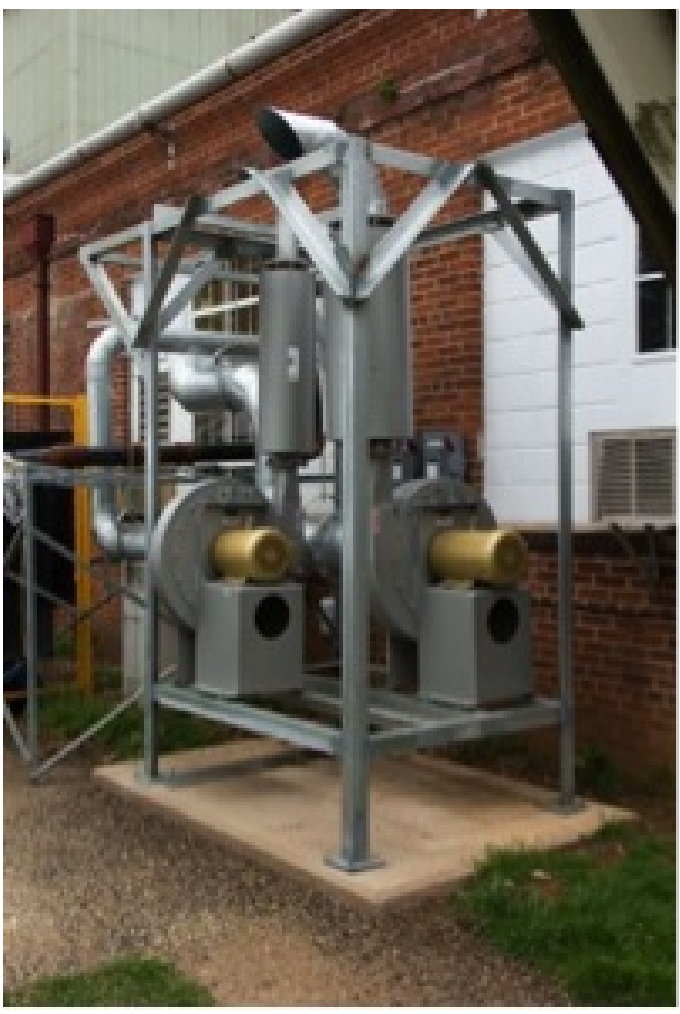

(b) Air handling facility

Figure 2.3: Photographs of UVa IPS Facility showing a) the IPS research tunnel and b) the air handling facility with the scavenge leg blower on the left and the core leg blower on the right

An ejector nozzle was added to the end of auger to aerate the particles to avoid clumping, figure 2.5. The ejector consists of two parts: 1) a metal tube $1^{1 / 8}$ inches in diameter with a port milled in the side to slip on to the end of the auger and 2) an air nozzle (McMaster Carr part number 8451K31) that is positioned above the auger, but still within the ejector tube. To ensure that the particles were fully dispersed, several different air nozzle pressures were tested for the PDS as shown in figure 2.6. At low pressures, e.g. 0-5 psi, clumps of particles are readily discerned but are generally replaced with a fine cloud of individual particles for pressures of 10 psi or greater. Based on these experiments, a pressure of 20 psi was chosen as a baseline condition as this ensured complete dispersion while minimizing the jet intensity. 


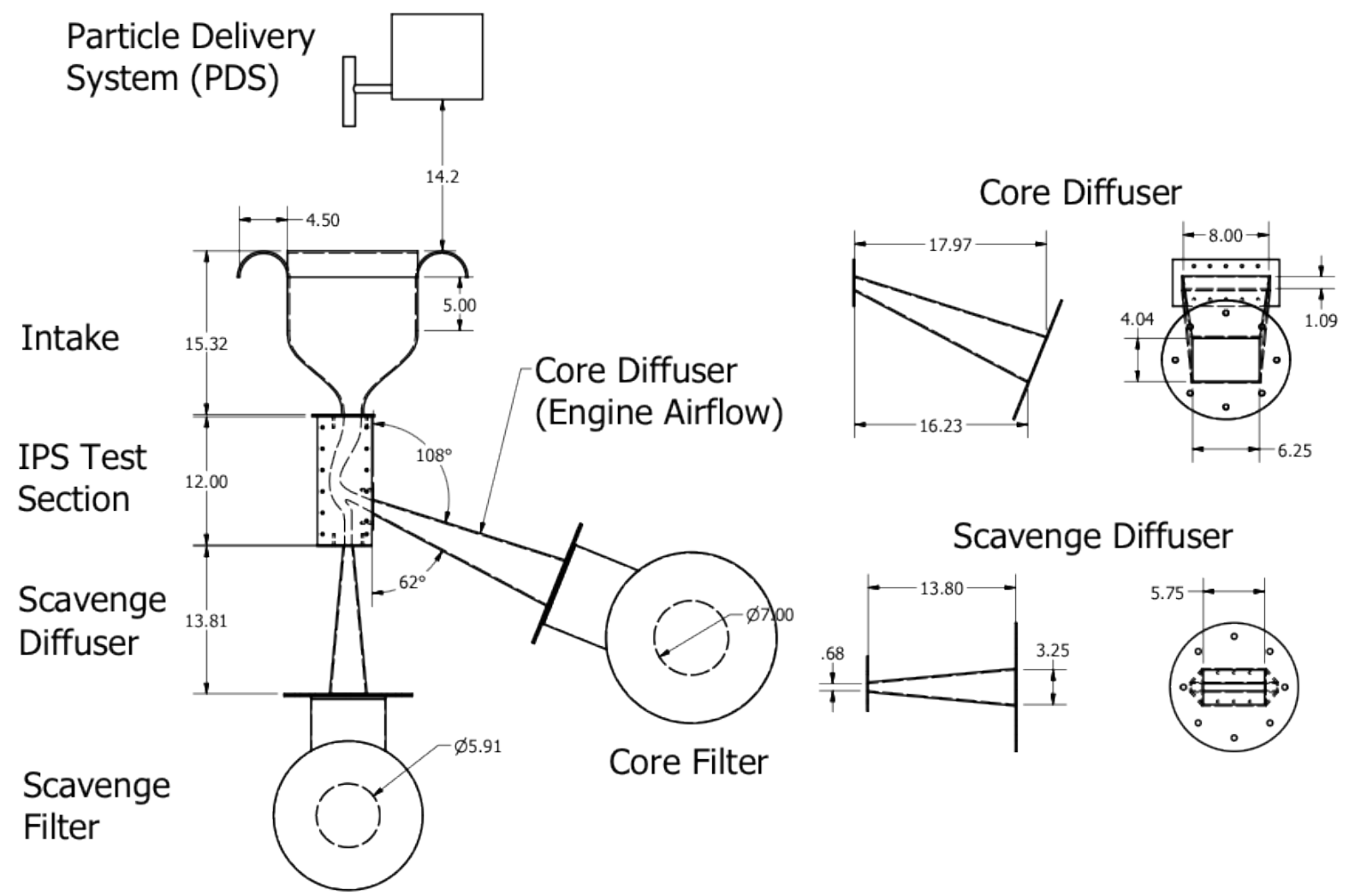

Figure 2.4: IPS research facility schematic showing the Particle Delivery System, air intake, IPS test section, diffusers, and particle filters. All dimensions are in inches

Minimizing the jet intensity results in lower turbulence that could be carried into the wind tunnel intake; according to Jiang et al. ${ }^{[20]}$, the effect on the particles should be minimal. Additionally, the ejector pressure and the variable feed rate of the augur may be used to study the effects of particle inlet conditions on IPS performance.

\section{Air Intake}

To obtain the low-turbulence uniform flow in the test section, an air intake that gradually contracts the flow area is required. ${ }^{[21]}$. Bell and Mehta ${ }^{[22]}$ outline a process on designing inlet nozzles to prevent flow separation and derive a polynomial that the contour of the nozzle 


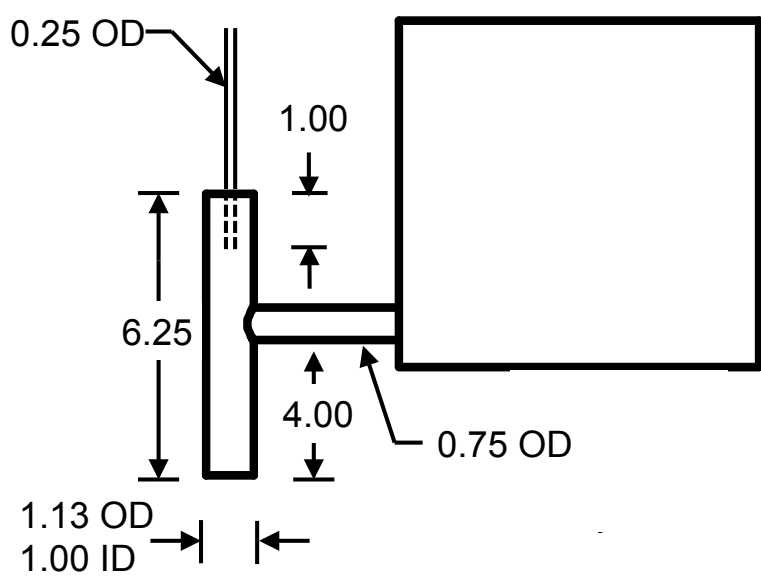

(a) PDS schematic

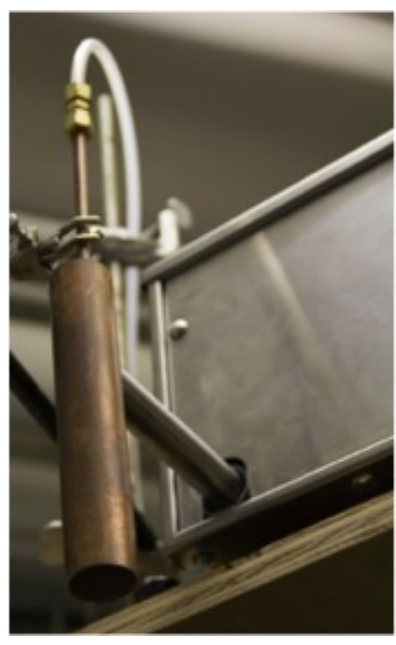

(b) PDS picture

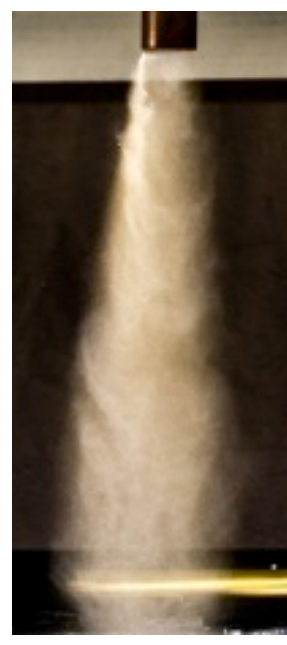

(c) PDS operation

Figure 2.5: Particle Delivery System, an accurate 106M screw feeder with attached aeration nozzle: a) PDS schematic b) PDS picture, and c) particle aeration operation
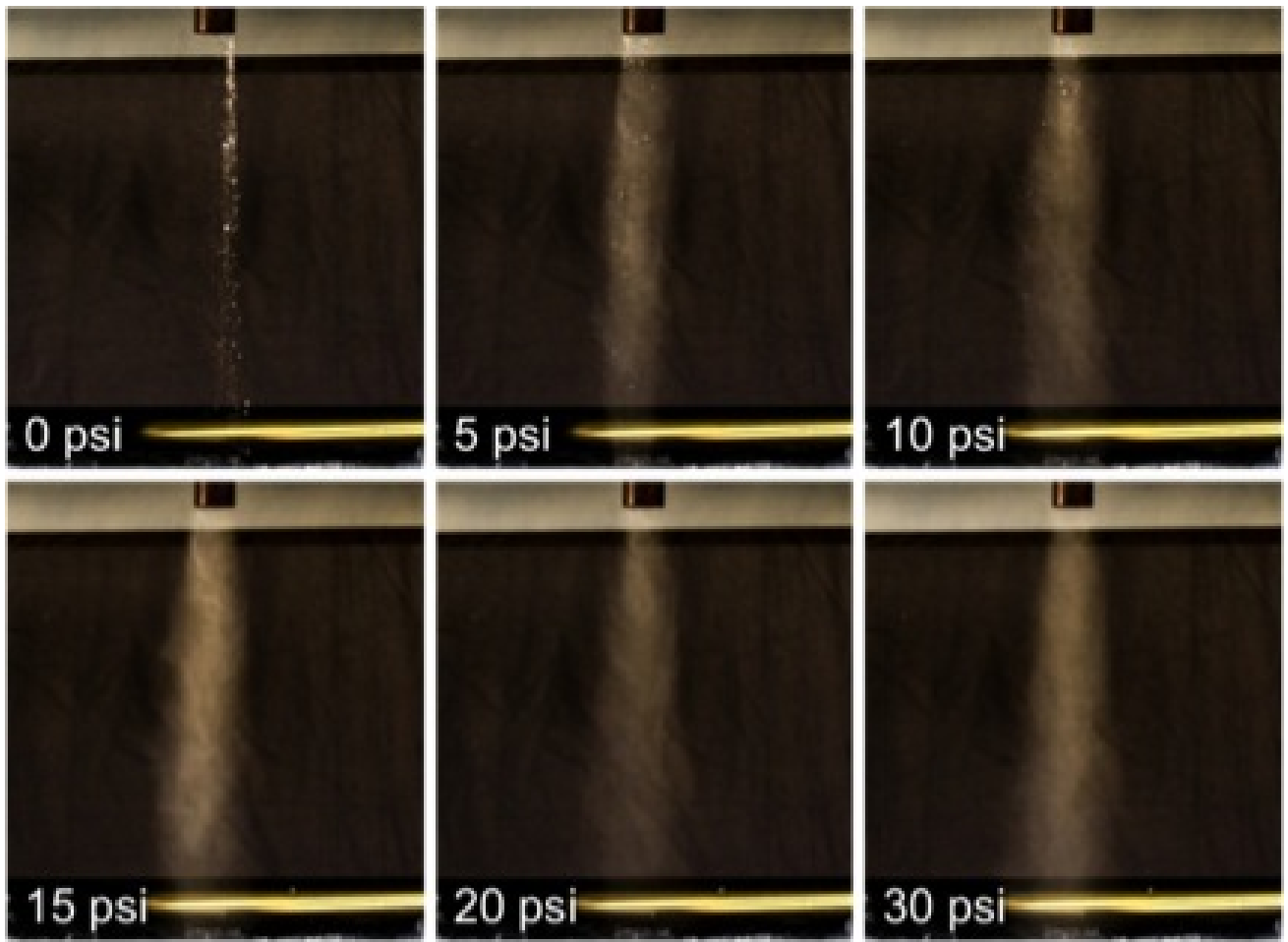

Figure 2.6: Influence of air pressure on PDS aeration; sand aeration was explored for varying nozzle exit pressures 


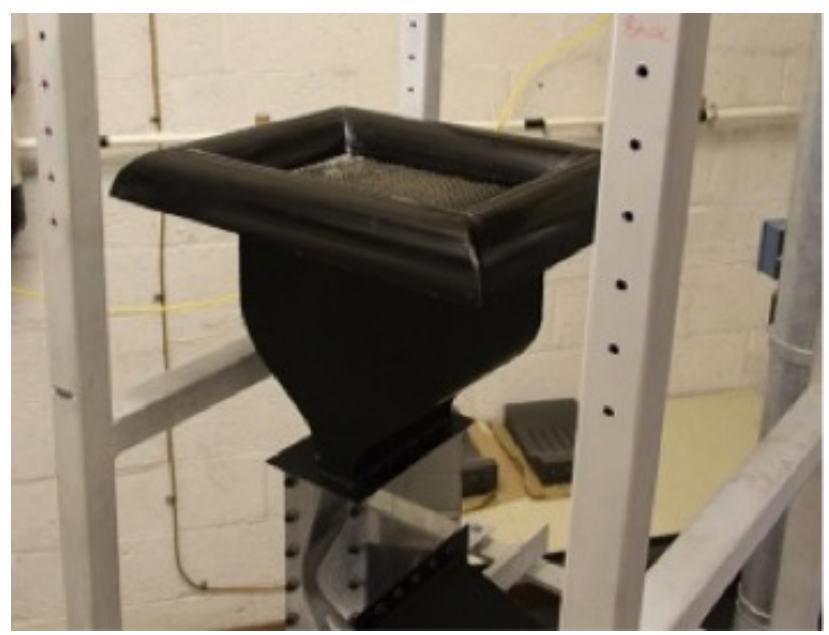

Figure 2.7: Air Intake with $1 / 2$ inch diameter, 3 inches long, hex-cell flow straightener installed (Aspect ratio of 6 )

should follow. Prior to this converging section, the beginning of the intake includes a 4.5 inch radius of curvature lip to avoid flow separation followed by a straight section to accommodate a 3 inch thick aluminum flow straightener made up of $1 / 2$ inch diameter hex-cells, resulting in a straightener aspect ratio recommended by Pope ${ }^{[23]}$. The resulting air intake with curved lip, honeycomb flow straightener and converging section is shown in figure 2.7.

\section{Test Section}

The rectangular geometry was designed to preserve the anticipated recirculation zone and pressure gradients found in the axi-symmetric geometry. As mentioned above, using a channel height and mean velocity consistent with a full-scale IPS system eliminated scaling issues. Figure 2.8 shows the resulting design for the test section consisting of five parts: the hub, splitter, outer surface geometry (OSG) and two sidewalls. The OSG is designed to be modular to allow interchangeable surfaces. The modular construction also allows for access for oil-streak flow visualization and cleaning of any particulate lodging and buildup. Coordinates used to define the geometry are given Table 1. 


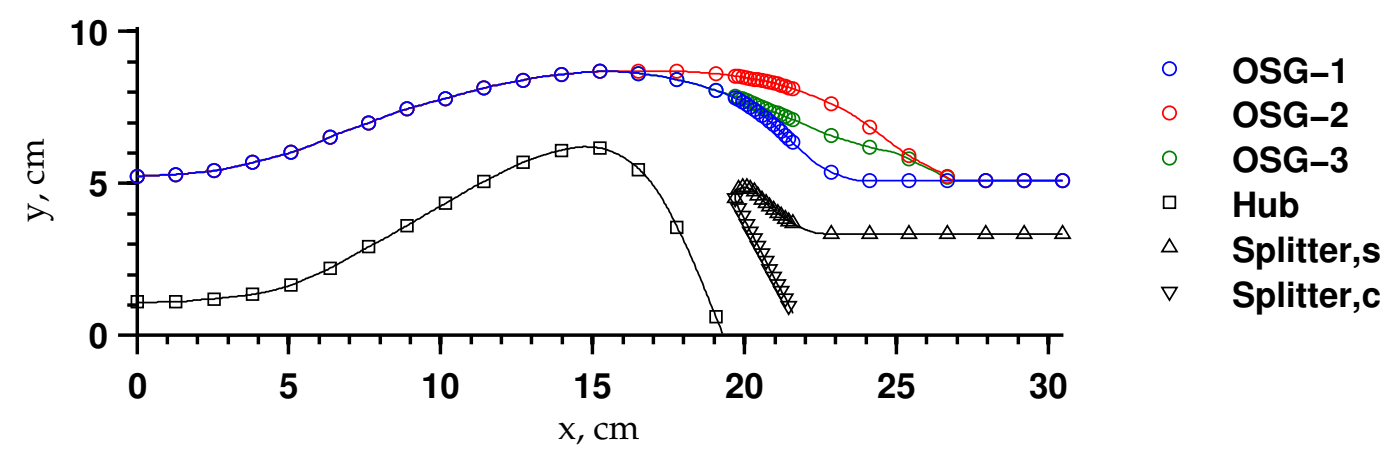

(a) Test section coordinates

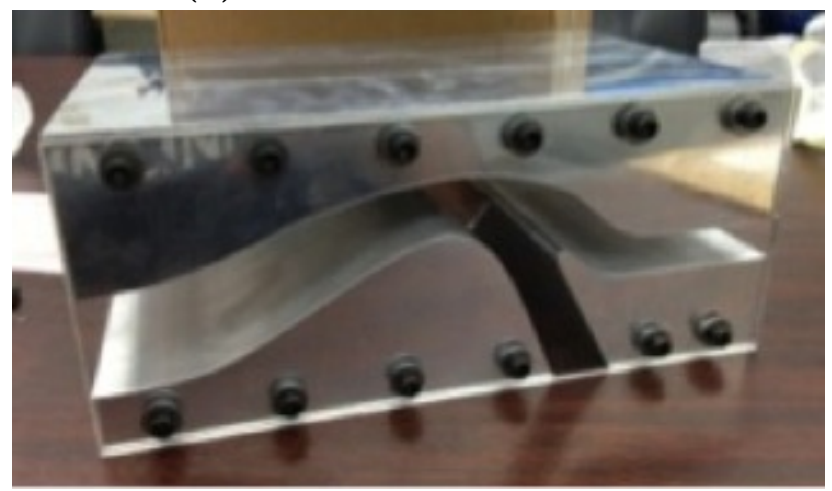

(b) Test section picture

Figure 2.8: Test section with OSG-1, with a) schematic of the test section geometry (see Table 1) and $\mathrm{b}$ ) picture of the test section. The inflow cross section is $8.00 \times 1.63$ inches and the total length is 12 inches.

\section{Outlet Diffusers}

To slowly increase the flow line cross-sectional areas so that they are consistent with the filter inlets (discussed below), the core and scavenge streams from the test section both enter diffusers. These diffusers were designed to allow them to slow down the air sufficiently without allowing significant flow separation, thus reducing the pressure losses and thereby power requirements of the blower system. Avoiding flow separation in the diffusers is also important as this can cause unsteady pressure oscillations that can feed back into the test section. The diffuser performance map given in White ${ }^{[24]}$ was used to design them to be 
as compact as possible while avoiding any flow separation. This was done by selecting the desired inlet and outlet areas of the diffuser (limited by the test section outlet and filter inlets respectively) and determining the maximum divergence angle while maintaining acceptable pressure recovery.

\section{Particle Filters}

After the flow exits the diffusers, the particles are collected so that they may be weighed in order to determine the sand separation efficiency. To be able to adequately remove all of the particles from the air, a set of air filters designed for large diesel engines were chosen (Donaldson EBB B160049 for the core flow and B140044 for the scavenge flow). These filters have a stated efficiency rating of $99.9 \%$ for dust and calibration in our facility has shown them to be at least $99 \%$ effective for the flow rates and test dust used in this study. A high precision weight measuring scale is used for PDS and filter weighing, a Sartorious Signum 1, with $0.1 \mathrm{~g}$ resolution, repeatability of $0.08 \mathrm{~g}$, and a maximum capacity of $35 \mathrm{~kg}$. The high-sensitivity and high-accuracy scale was important to minimize uncertainty associated with particle separation efficiency as will be discussed later.

\section{Air Handling System}

To simulate these effects, two industrial blowers are used which exhaust into ambient air. The upstream lines of these blowers thus provide sub-atmospheric pressure to pull flow independently through the core flow and scavenge flow legs. In order to limit the size of the air handling system required, the test section kept the same height and mean flow velocity but employed a span-wise length of 8 inches. This allowed a high aspect ratio of 8:1 at the throat to satisfy sixth facility requirement. The test section was designed for Mach 0.4 flow at the throat; resulting in a mass flow rate to $2 \mathrm{lbm} / \mathrm{s}$ thorough the test section intake. 
To adequately size the blowers, the pressure loss of each component must be estimated (Table 2). Using the design specifications of the IPS, the pressure loss in the test section was calculated to be on the order of $2 \%$ of the total pressure for the core leg and $5 \%$ for the scavenge leg. Loss in the outlet diffusers was calculated based on the pressure coefficient hart given by White ${ }^{[24]}$. Losses in the particle filters were reported from Donaldson at the specified flow rate for each leg. The air handling system losses were estimated based on head loss calculations given by White for piping and turns. In addition to the estimated pressure losses, a $20 \%$ safety factor was included to mitigate the risk of under sizing the system. As a result, two blowers from Cincinnati Fan were selected to meet the mass flow and pressure loss requirements, a model HP-8E24 with a $15 \mathrm{HP}$ motor for the core flow leg and a model HP6E-26 with a $7.5 \mathrm{HP}$ motor for the scavenge flow.

Each blower is controlled by a variable frequency drive, allowing ease of control of mass flow split between the core flow and scavenge flow. The scavenge flow split, $\beta$, is defined as the ratio of scavenge air to the total amount of air that enters the inlet, Eq. (1).

$$
\beta \equiv \frac{\dot{m}_{s}}{\dot{m}_{t}}=\frac{\dot{m}_{s}}{\dot{m}_{s}+\dot{m}_{c}}
$$

The flow rate though each blower are measured using Tek-Air VT5000 Vortek flow stations. The flow stations have a reported volumetric flow rate accuracy of $2 \%$. With the tunnel installed, the core and scavenge blowers demonstrated the required $2 \mathrm{lbm} / \mathrm{sec}$ and $0.5 \mathrm{lbm} / \mathrm{sec}$ respectively plus adequate margin. 


\subsection{Surface Flow Visualization Results}

Oil-streak surface visualization describes flow direction and local shear strength, including flow separation regions, which in turn can be used to infer characteristics of the flow away from the surface. Surface oil streaks can be obtained by suspending particles in an oil base and applying the mixture to the test article surface with a fine brush, shortly before tunnel operation. The oil mixture will then streak as the air moves within the flowpath ${ }^{[25]}$. For this study, kerosene is used as the oil, titanium dioxide as the particles, and oleic acid is used as a surfactant.

Oil-streak experiments were completed for OSG-1 with various intake configurations (with and without the flow straightener and with and without the PDS air supply) the results are shown in figure 2.9. The core and scavenge flow rates were held constant with a core mass flow of $1.71 \mathrm{lbm} / \mathrm{sec}$ and $\beta=0.10$ (representative conditions for IPS operation). For all cases, the air entering the test section is well behaved with straight lines indicating uniform steady flow. The oil streaks on the OSG indicate a clear separation zone near the scavenge entrance, as expected, where the oil streak mixture is has no discernible movement downstream. The flow acts two dimensionally down the center of the test section, but there is evidence of three-dimensional effects near the walls. It is supposed that the interaction between the recirculation zone and the wall are causing the vortices to appear in the recirculation zone, similar to those seen in other experiments26. These structures point to effects that have not been explored previously in IPS systems. For the baseline case, the vortical structures present near the end walls account for less than $30 \%$ of the total cross sectional area. This is in line with design requirement (6) for having a significant area of two-dimensional flow and an area with strong wall effects for study.

To determine the effects of the PDS ejector air on the tunnel a second oil-streak test was 

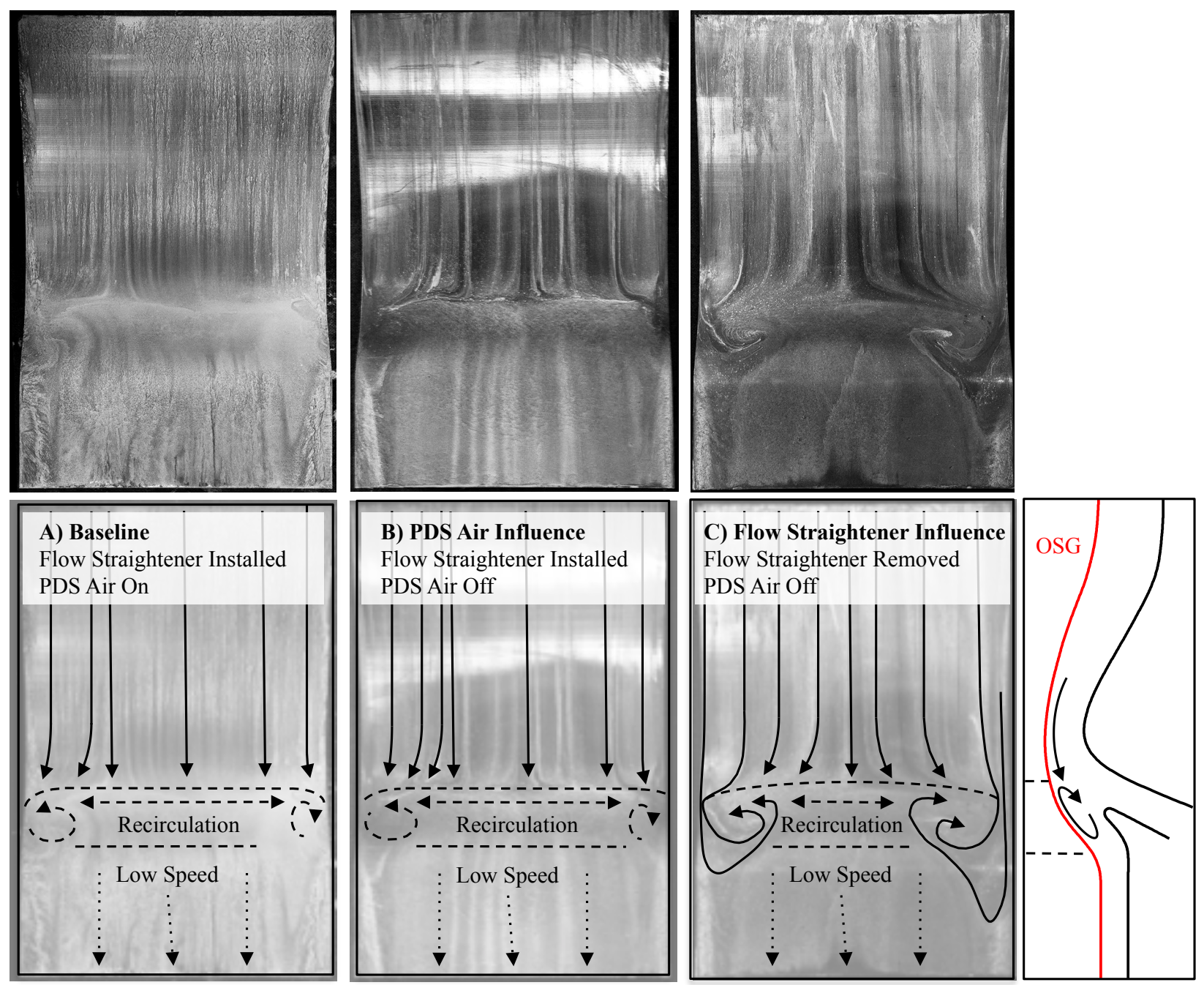

Figure 2.9: Oil-streak visualization of the OSG-1 flow path with three different intake configurations. Each case contains the original image on top and an annotated image below. From left to right: OSG-1 side view, case a) Baseline operation with the flow straightener on and the PDS air running, case b) Operation without the PDS air, and case c) Operation without the flow straightener installed and without the PDS air. For each case, the core flow rate is $1.71 \mathrm{lbm} / \mathrm{sec}$ and $\beta=0.10$

performed with the PDS air turned off. There is little change in the results due to the PDS air. This confirms that the selection of the 20psig setting for the PDS ejector air will not adversely change the flow field.

It is possible the flow straightener may affect the particles as they enter into the tunnel 
intake. It was therefore desired to see how the flow in the test section would change if the flow straightener was absent. The results show that there is a significant change when the intake flow straightener is absent. The sidewall vortices increase in size and account for at least $65 \%$ of the cross sectional area, compared to $30 \%$ in cases (a) and (b). It is believed that the increase in three-dimensional effects are caused by separation in the tunnel inlet due to the lack of flow straighteners that keep the flow attached around the inlet lip. This result requires us to maintain use of the flow straighteners for further experiments in the facility.

Oil-streak results for the tunnel where originally presented at the 28th AIAA Aerodynamic Measurement Technology, Ground Testing, and Flight Testing Conference ${ }^{[8]}$. These results where incorrectly reported with a scavenge split of 0.16 , the actually scavenge split was 0.10 . Figure 2.10 shows a comparison of a) oil-streak results with $\beta=0.10$ and b) oil-streak at $\beta=0.16$. The comparison of the two cases shows that the flow field does not significantly change on the surface of the IPS.

Figure 2.11 shows the results of the oil streak on the hub geometry for the baseline conditions, corresponding to fig 2.9; only one condition is shown because there is no remarkable difference in the hub surface flow between the three cases. As the flow makes the sharp turn into the core flow leg, flow separation near the walls can be seen. This separated region accounts for approximately $24 \%$ (12\% on either side) of the flow field as it leaves the test section and enters the diffuser. Overall, the oil streaks show that the majority of the flow is two-dimensional for the baseline case. 

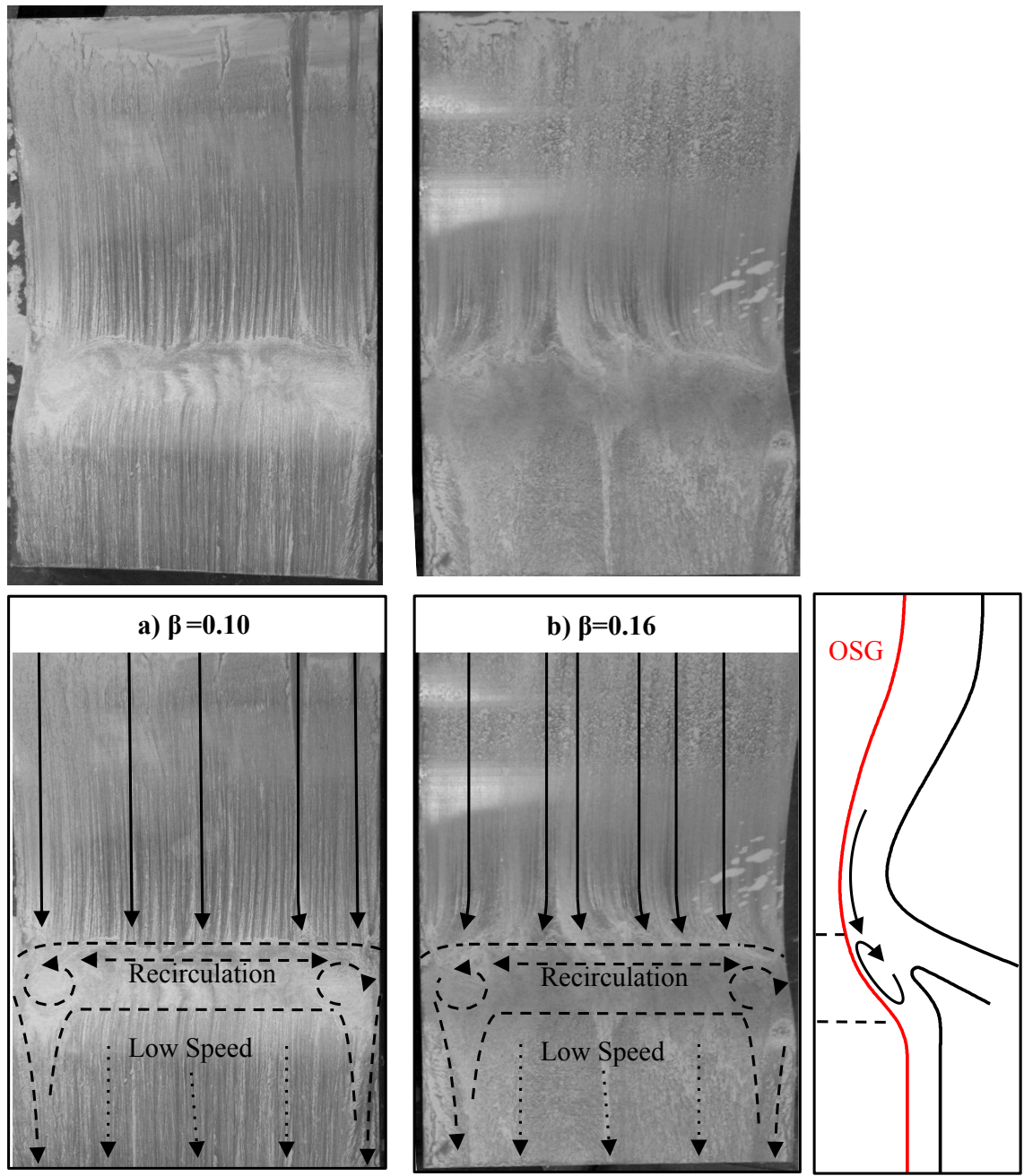

Figure 2.10: Oil-streak visualization of the OSG-1 flow path for scavenge split values of $\beta=0.10$ and $\beta=0.16$. There is little difference between the case of a) $\beta=0.10$ and b) $\beta=0.16$ in the location of the recirculation zone and the presence of the corner wall vortices.
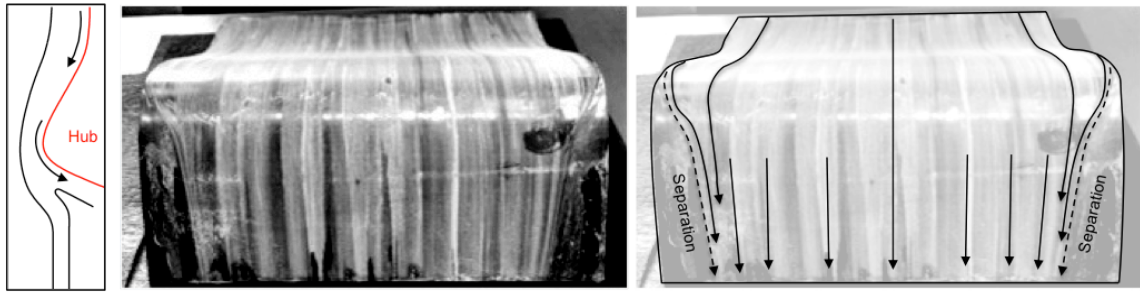

Figure 2.11: Oil-streak visualization of the core side of the hub for the OSG-1 flow path. Core flow rate of $1.71 \mathrm{lbm} / \mathrm{sec}$ and $\beta=0.10$ 


\subsection{Separator Efficiency}

The ultimate performance of the separator can be judged by its ability to eliminate particulate from the core air stream. The separator efficiency, $\eta$, can be defined as the ratio of scavenge particle mass, $\dot{m}_{s}$, to the total ingested particle mass, $\dot{m}_{t}$, as shown in Eq. 4.2. The total ingested particle mass can be determined by to total mass captured by both the scavenge $\left(\Delta m_{s f}\right)$ and core $\left(\Delta m_{c f}\right)$ filters; as shown later, the uncertainty of this measurement can be evaluated using the mass delivered by the particle delivery system.

$$
\eta \equiv \frac{\dot{m}_{s}}{\dot{m}_{t}}=\frac{\Delta m_{s f}}{\Delta m_{s f}+\Delta m_{c f}}
$$

The mass of particles delivered by the PDS system is measured for each experiment as is the particulate mass that accumulate in the barrier filters installed downstream of the test section on both the core and scavenge flow legs (corrected for filter efficiency). Ideally, the total mass in the filters should equal that placed in the PDS. The specific procedure used to compute the separation efficiency is discussed below.

For each efficiency measurement both barrier filters are removed from the tunnel and weighed; the PDS, filled with particles, is also weighed. Everything is then re-installed and the tunnel flow rates are brought up to the desired operating condition. Once the steady airflow operating condition is reached, the PDS is turned on and the tunnel is allowed to run for predetermined length of time in order to have enough particle buildup in the barrier filters to achieve an accurate measurement. The PDS is then turned off, and after particle delivery has stopped, the air system is turned off as well. Both barrier filters and the PDS are then weighed in order to determine the change in mass of each component.

Generally, a small fraction of particles are lost during the experiments. On a typical run, 
about 100 grams of particles are delivered into the airstream and 1-2 grams are not picked up in the filters. This lost mass is defined herein as the residual mass $\left(\Delta m_{r}\right)$. This can be partially traced to a small amount of particles that deposit on the surfaces of the tunnel. In addition, very small particles may pass through the filters or can be lost to the ambient air between the PDS and the tunnel inlet as a result of excessive aeration. While these are small losses, it is important to quantify their effect.

The uncertainty determined from Taylor ${ }^{[26]}$ of the scavenge split and separation efficiency are shown in Eqs. (3) and (4) respectively.

$$
\delta \beta=\sqrt{\left(\frac{\dot{m}_{s}}{\left(\dot{m}_{c}+\dot{m}_{s}\right)^{2}} \delta \dot{m}_{c}\right)^{2}+\left(\frac{\dot{m}_{c}}{\left(\dot{m}_{c}+\dot{m}_{s}\right)^{2}} \delta \dot{m}_{s}\right)^{2}}
$$

The uncertainty, $\delta \beta$ in the scavenge flow split is calculated with the error in each of the legs, $\delta \dot{m}_{c}$ and $\delta \dot{m}_{s}$, taken as the $2 \%$ reading error reported in the flow meter documented calibration.

$$
\delta \eta=\sqrt{\left(\frac{\Delta m_{s}}{\left(\Delta m_{c}+\Delta m_{s}\right)^{2}} m_{r}\right)^{2}+\left(\frac{\Delta m_{c}}{\left(\Delta m_{c}+\Delta m_{s}\right)^{2}} m_{r}\right)^{2}}
$$

Efficiency uncertainty, $\delta \eta$ is calculated with the measurement uncertainty taken as the residual $\left(m_{r}\right)$ between the mass of sand input through the PDS and the mass of sand captured in both of the filters, this difference is between $1 \%$ to $2 \%$ of the input sand. It is difficult to quantify the uncertainty of the individual core and scavenge measurements, but both are less than the residual; therefore, the residual is used in their place. The accuracy of the balance is not included, $\delta m_{b}$, because its contribution to the error is less than $0.05 \%$ of the input sand and is negligible compared with the sand is lost in the system.

As with the oil-streak, the initial results previously presented were reported with an error in the scavenge split. Corrected results are shown in Fig. 12 for the OSG-1 flow path 
using A4 Coarse Test Dust. The core flow was held constant at $1.71 \mathrm{lbm} / \mathrm{sec}$ at three target scavenge splits of $\beta=0.14,0.16$, and 0.20 . The measured efficiencies fall with the expected range of values given by Filippone and Bojdo ${ }^{[10]}$. It is evident that the efficiency has a strong dependence on the scavenge split for OSG-1. As $\beta$ is increased, the efficiency of the system is also increased; this implies that there are significant fluid effects on the particles within the IPS.

\subsection{Conclusions}

A facility has been designed and built to study the complex flows associated with inertial particle separators. The tunnel has demonstrated its ability to reproduce the mass flow and particle conditions that are seen in IPS systems and can vary the mass flow of the core and the scavenge flow legs independently, allowing for complete control of total mass flow and scavenge split. Oil-streak measurements of the test section show a substantial region of 2-dimensional flow with areas near the walls demonstrating significant 3-dimensional regions for further study. The two dimensional IPS operates at efficiencies within the expected range and demonstrated trends previously observed and has demonstrated the capability to measure overall particulate separation efficiency with less than $2 \%$ error. 


\section{CHAPTER 3}

\section{Efficiency of an Inertial Particle Separator}

\subsection{Introduction}

When a turbine engine ingests sand or dust, the components of the engine may undergo highly damaging erosion, and the engine life can be significantly reduced ${ }^{[8-11,16]}$. This presents a major readiness, cost, and maintenance issue for military and civilian missions during low altitude operations in sandy areas such as the Middle East ${ }^{[11]}$. Sand and dust separation devices traditionally fall into the three categories: Vortex Tube Separators (VTS), Inlet Barrier Filters (IBF), and Inertial Particle Separators (IPS). In contrast to VTS and IBF systems that are normally applied as airframe installed options, IPS systems are currently designed as integral to the engine. In many cases an integrated design can result in a more compact system with lower total pressure losses.

The basic principle behind IPS design is that particle-laden air enters the annular inlet, is turned, and then bifurcated by a splitter. This causes the main core flow to rapidly turn while the scavenge flow continues in an approximately strait direction allowing the inertia of 


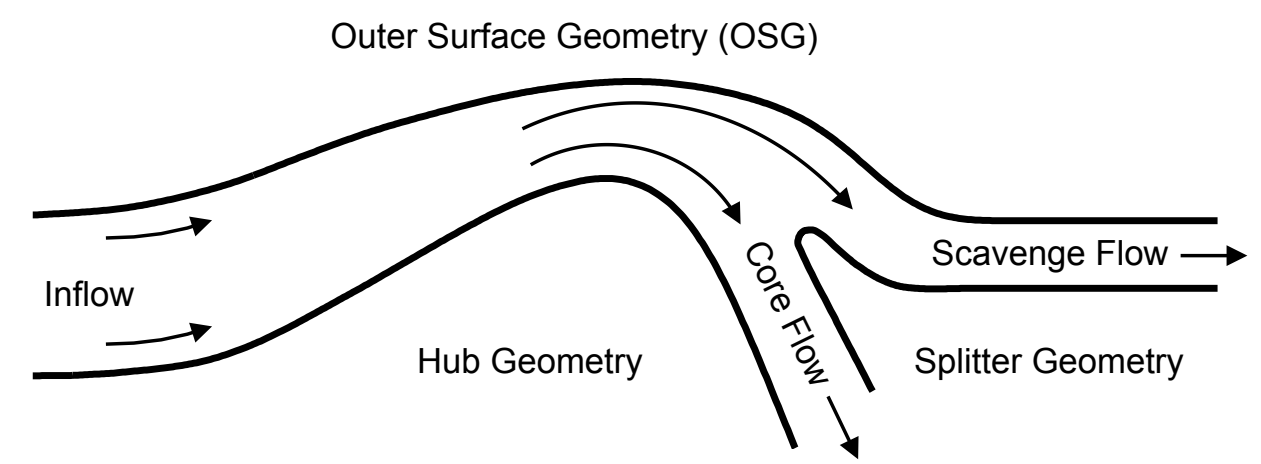

Figure 3.1: Inertial particle separator geometry

the particles to be used for separation. In an installed IPS system, the mass flow of air to the core flow is driven by the turboshaft engine and the split to the scavenge flow is driven by some type of blower or ejector system.

A simplified schematic that identifies the core and scavenge paths is shown in figure 4.1. Within the separator, the airflow makes a hub-side turn while the scavenge flow continues with very little flow turning. While very small drag-dominated particles will tend to follow the streamlines, moderate size particles with sufficient inertia follow the scavenge path which prevents them from following the core airflow around this sharp corner. Large particles that are inertia-dominated will only be weakly effected by the flow and will be controlled more by their initial trajectories and any bouncing off of solid surfaces. In general, the hub, splitter, and Outer Surface Geometry (OSG) are ideally designed to ensure that most of the particles enter the scavenge flow leg, so that the core flow is clean of damaging particulate. The scavenged particles are then ejected from the aircraft via the scavenge flow and the cleaned air proceeds to the turbine engine.

For design of a high efficiency low pressure loss IPS, a fundamental understanding of the multiphase flow physics is required; particularly, the relationship of the airflow bifurcation characteristic effects on dust particles. Understanding of both the fluid flow field and 


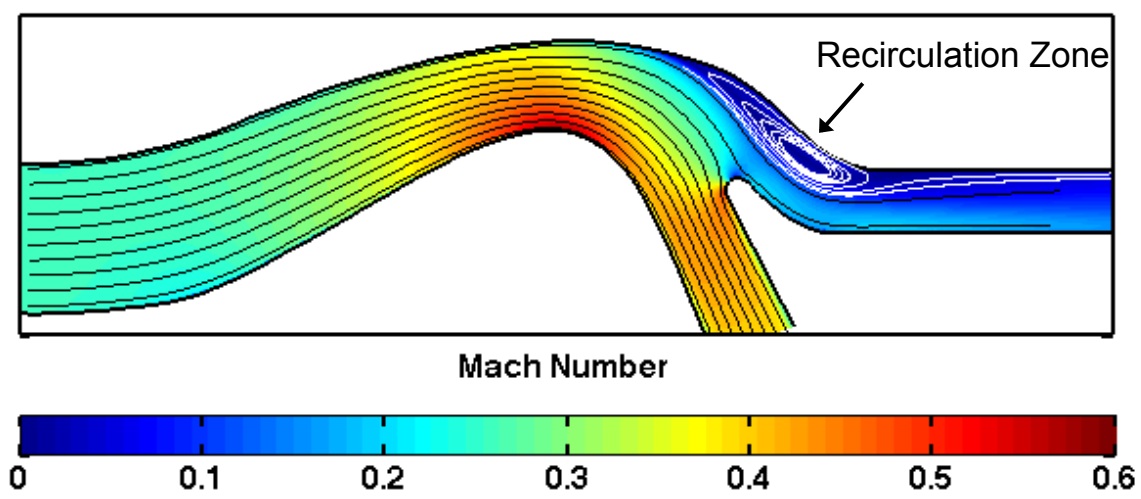

Figure 3.2: Qualitative Mach contours and streamlines to illustrate the recirculation zone present in the scavenge flow.

the particulate motion in the splitter region is critical to achieve designs that maximize particle separation efficiency. A significant amount of effort has gone in to the computational studies $^{[7,14-20]}$. While these studies have identified features like the recirculation zone using solutions to the Reynolds Averaged Navier-Stokes equations, they have focused primarily on the average flow field and do not account for dynamic flow structures and the effect that these structures have on particles.

The particular region of interest being addressed here is a recirculation zone that exists along the outer surface of the flow path near the splitter, Fig 3.2. It is believed that this flow feature has a significant impact on separator efficiency; however, the details of the flow structure are poorly resolved by averaged flow solution techniques. The direct application of full three dimensional direct numerical flow simulation for the geometries is highly resource intensive and often out of scope for application to the problem. While computational simulations can allow quantitative results, such unsteady three-dimensional turbulent flow solutions performed for an IPS geometry are computationally expensive.

Similarly, the particle dynamics of an irregularly-shaped polydisperse mixture that includes significant wall reflections are not well modelled. Thus, prediction of separation efficiency 


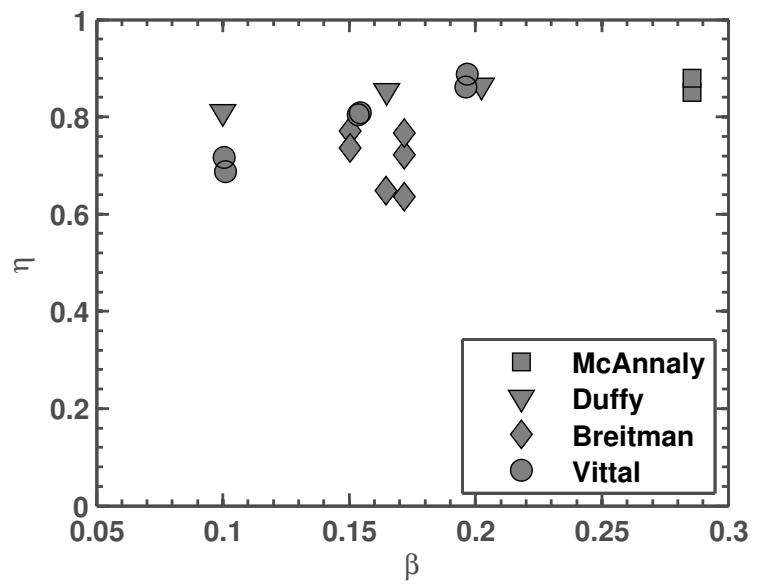

Figure 3.3: Previous IPS efficiency data relevant to current IPS research

and an understanding of the controlling flow phenomena influencing the particulate motion associated with the IPS are only definitively obtained with an experimental approach. As expressed by Jiang et al. in Ref ${ }^{[20]}$, experimental data is needed to validate the computational models.

To date, previous test facilities have only been designed to quantify the overall performance of IPS designs as shown in figure $3.3^{[1-4]}$. Duffy ${ }^{[2]}$ in particular did an extensive survey of three different concepts over a wide range of operating conditions. These studies have looked at the overall efficiency and aerodynamics of the particle separators with regard to installed performance, which can be used to verify the end result of a model, but provides little data for model validation.

The goal of this work is to begin the work required to understand the flow physics present in an IPS and particularly that of the recirculation zone. Oil-streak visualization is employed to qualify the position of the recirculation zone along the outer surface geometries. Efficiency measurements of the IPS geometries are compared to show the effect of the recirculation zone. This work will begin to fill the void of experimental data with a simple well defined geometries with experiments over a range of conditions. 


\subsection{Methodology}

A facility was designed to create the specific branching flow conditions present in an IPS system, a detailed description can be found in previous work ${ }^{[8]}$. The system is capable of several measurements, including overall sand separation efficiency, surface flow visualization, and quantitative optical internal flow diagnostics. This work will focus on separation efficiency and surface flow visualization; optical measurements will follow with planned experiments to quantify the fluid flow field and particle paths through the IPS. A schematic of the tunnel is shown in figure 3.4. The main components of the tunnel (in flow order) are 1) the particle deliver system (PDS), 2) the tunnel intake, 3) the IPS test section, 4) core and scavenge flow diffusers, 5) core and scavenge particle filters, and 6) core and scavenge centrifugal blowers (not shown).

The facility has the ability to independently control the mass flow rate through the scavenge and core flow paths using two independently controlled centrifugal blowers. Each blower is controlled by a variable frequency drive, allowing ease of control of mass flow split between the core flow and scavenge flow. The scavenge flow split, $\beta$, is defined as the ratio of scavenge air to the total amount of air that enters the inlet, Eq. (1).

$$
\beta=\frac{\dot{m}_{s}}{\dot{m}_{c}+\dot{m}_{s}}
$$

The mass flow rate through the system is set using a closed loop Proportional-IntegralDerivative (PID) controller. This controller sets a constant mass flow rate through the core flow leg, then adjusts the scavenge flow to achieve the desired flow split.

The facility features a modular test section capable of performing measurements on different IPS geometries. Three different OSGs have been created to test the effects of the 


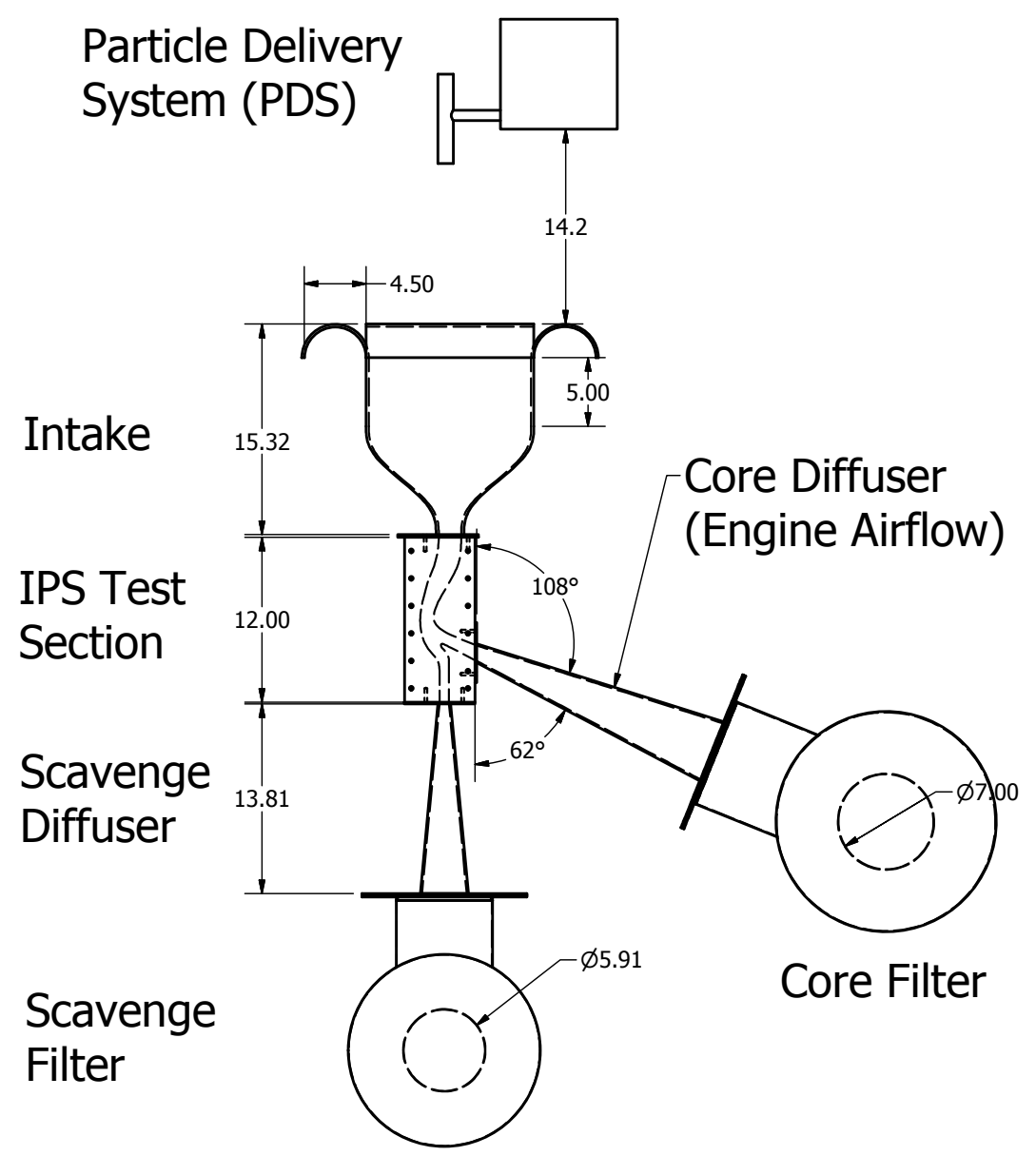

Figure 3.4: Facility schematic, dimensions are in inches

recirculation zone on separation efficiency. The three geometries are shown in figure 4.3, a detailed table of characteristic points is given in the appendix. The test section is 8 inches wide and is designed for Mach 0.4 flow at the throat $(x \approx 6$ inches $)$. These three OSGs have been selected to produce differences in the location of the recirculation zone. 


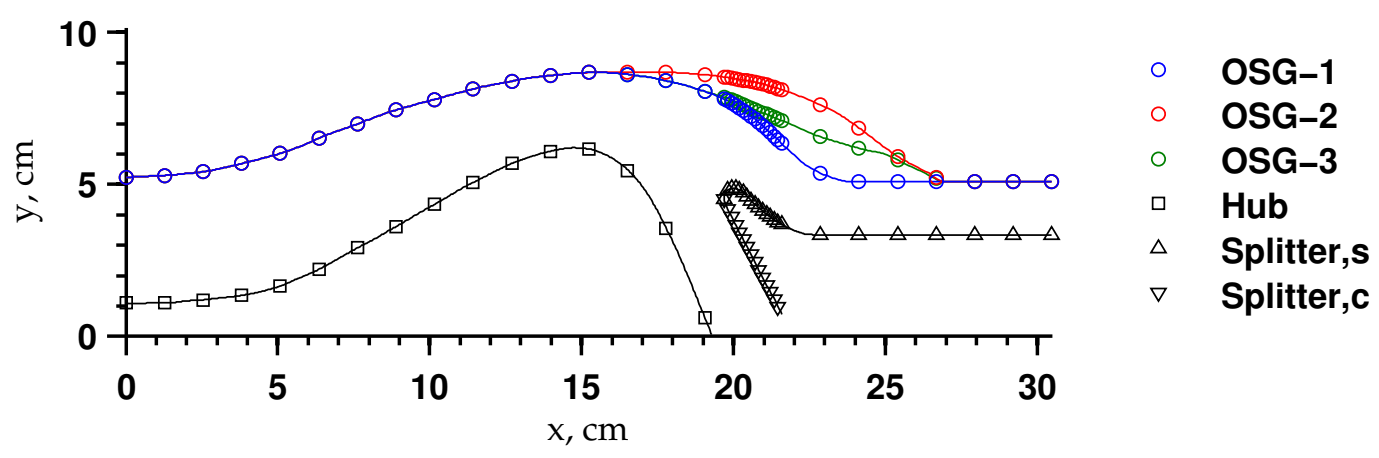

Figure 3.5: Test section geometries

\subsubsection{Flow Visualization}

To determine the location of the recirculation zone, oil-streak surface visualization was used. Oil-steak surface visualization describes flow direction and local shear strength, including flow separation regions, which in turn can infer characteristics of the flow away from the surface. Surface oil streaks can be obtained by first suspending particles in an oil base and applying the mixture to the test article surface,with a fine brush shortly before tunnel operation. The oil mixture will then streak as the air moves within the flowpath ${ }^{[25]}$. For this study, kerosene is used as the oil, titanium dioxide as the particles, and oleic acid is used as a surfactant. A photograph is then taken of resultant oil streaks for analysis and as a record.

\subsubsection{Efficiency Measurement}

Overall IPS efficiency is the target of this research, so it is intuitive to measure particle separation efficiency in the research facility. For each efficiency measurement both barrier filters are removed from the tunnel and weighed; the PDS, filled with particles, is also weighed. Everything is then re-installed and the tunnel flow rates are brought up to the desired operating condition. Once the steady airflow operating condition is reached, the PDS 
is turned on and the tunnel is allowed to run for predetermined length of time in order to have enough particle buildup in the barrier filters to achieve an accurate measurement. The PDS is then turned off, and after particle delivery has stopped, the air system is turned off as well. Both barrier filters and the PDS are then weighed in order to determine the change in mass of each component. Generally, a small fraction of particles are lost during the experiments. On a typical run, about 100 grams of particles are delivered into the airstream and 1-2 grams are not picked up in the filters. This lost mass is defined herein as the residual mass, $\Delta m_{r}$. This can be traced to particles that deposit on the surfaces of the tunnel, very small particles that pass through the filters, or particles that are lost to the ambient air between the PDS and the tunnel intake. While these are small losses, it is important to quantify their effect.

For this work the efficiency of the IPS is defined as the mass of particles entering the scavenge flow $\dot{m}_{s}$ path relative to the total number of particles entering the intake, $\dot{m}_{t}$.

$$
\eta \equiv \frac{\dot{m}_{s}}{\dot{m}_{t}}=\frac{\Delta m_{s f}}{\Delta m_{s}+\Delta m_{c f}}
$$

Here the particles entering the scavenge flow is determined by the change in mass of the scavenge filter, $\Delta m_{s}$. The total mass entering the intake is measured using the sum of the particles collected in the scavenge and core filters. This measurement can be checked by using the total mass of particles being delivered to the system $\Delta m_{P D S}$, resulting the the residual mass measurement. The residual mass, $m_{r}$ can be used to calculate the uncertainty of the efficiency measurement, $\delta \eta$, as shown in Eq. 4.3 .

$$
\delta \eta=\sqrt{\left(\frac{\Delta m_{s}}{\left(\Delta m_{c}+\Delta m_{s}\right)^{2}} \delta m_{r}\right)^{2}+\left(\frac{\Delta m_{c}}{\left(\Delta m_{c}+\Delta m_{s}\right)^{2}} \delta m_{r}\right)^{2}}
$$




\subsubsection{Test Dust}

Particle motion in an IPS is composed mainly of two mechanisms, fluid forces (primarily drag) and the particle's inertial forces (primarily bouncing). Small particles which have a small relative mass, and therefore small inertial forces, will be dominated by fluid flow, whereas particles with large relative inertia will not be significantly affected by the fluid forces and their path through the IPS will be governed by bouncing. Typical sand and dust entering the engine will have particles covering the entire spectrum of particle sizes, ranging from totally fluid dominated particles to particles that are entirely bounce dominated.

The Stokes Number can be used to determine how the fluid and inertial forces will influence the motion of a particle. The Stokes Number is a non-dimensional parameter which is the ratio between a particle response time and a domain fluid response time. The particle response time, or how fast a particle reacts to the fluid forces imposed upon it, can be determined by the ratio of the effective particle momentum to the acting fluid forces Eq. 3.4.

$$
\tau_{p} \equiv \frac{m_{e f f}|\vec{w}|}{F_{D}}=\frac{\left(\rho_{p}+c_{\forall} \rho_{a i r}\right) d_{p}^{2}}{18 \mu_{\text {air }} f}
$$

The domain fluid response time is simply the time it takes the fluid to move across the domain, Eq. 3.5

$$
\tau_{D} \equiv \frac{D}{u_{D}}
$$

Taking the ratio of these two time constant results in the domain Stokes Number, Eq. 4.4

$$
S t_{D} \equiv \frac{\tau_{P}}{\tau_{D}}=\frac{\left(\rho_{p}+c_{\forall} \rho_{a i r}\right) d_{p}^{2}}{18 \mu_{a i r} f} \frac{u_{D}}{D}
$$




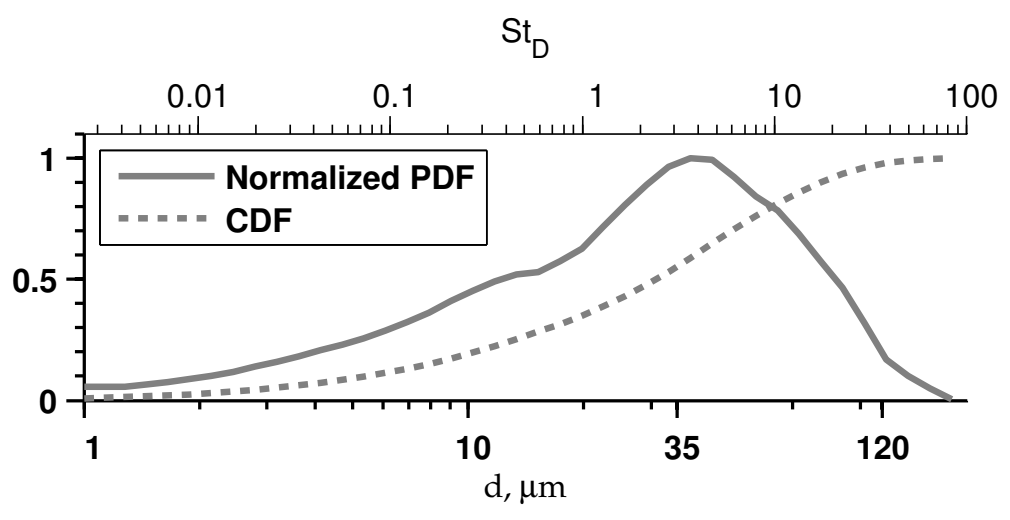

(a) Particle size distribution

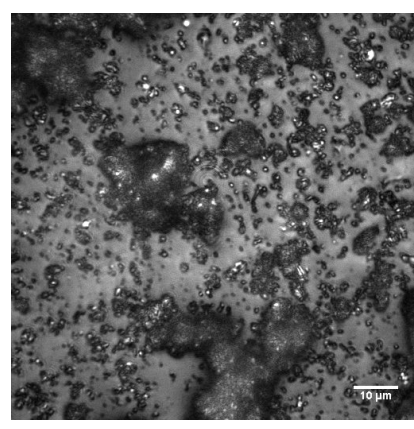

(b) Image taken with a confocal microscope at $100 \mathrm{x}$

Figure 3.6: A4 Coarse Test Dust

It is important to note that this domain Stokes Number, is a generalized value to give a particle's characteristic Stokes Number for the entire flow field. There are other Stokes Numbers, such as the turbulent Stokes Number that relate how a particle will react to small perturbations.

If a particle's response to the fluid is dominated by its own momentum forces, particle will have a longer response time relative to the fluid response time, resulting in a Stokes Number much larger than 1. Conversely, if a particle's response time is much shorter than the fluid's, it will have a Stokes Number much less than 1. A particle who's Stokes number is on the order of 1 is likely to have it's motion influenced by both bouncing and fluid forces.

Overall IPS efficiency measurements will be conducted using A4 Coarse, Arizona Test Dust (ISO 12103-1) from Powder Technology Inc. This is a standard test dust that can approximate the conditions seen by the system when installed on an aircraft. The test dust is composed primarily of Silica, $\mathrm{SiO}_{2}$, and Alumina, $\mathrm{Al}_{2} \mathrm{O}_{3}$. Particles cover a range from $0-200 \mu m$; the volume distribution is shown in figure 3.6a. Figure 3.7 shows the range of Stokes Numbers present in the test dust for the conditions being examined. This analysis shows that the IPS will have particles covering the entire regime of particle behavior. 


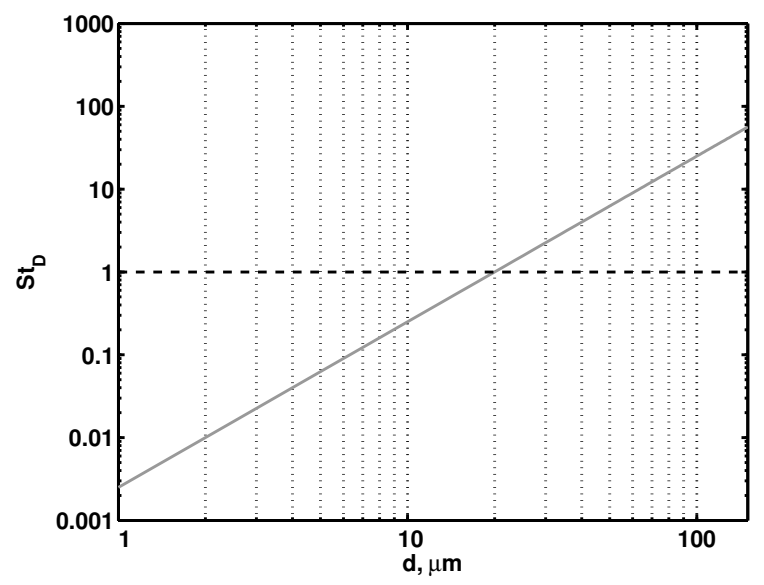

Figure 3.7: Test dust domain Stokes Number

The dust particles are of irregular shapes, evident in the picture shown in figure 3.6b. The irregular shape will effect the drag of each particle differently and will also effect how the particle bounces. It is impossible to generalize these effects in the Stokes Number calculation, but it is important to note its existence.

\subsection{Flow Visualization}

Oil-streak flow visualization was completed for each OSG at a scavenge split, $\beta$, of 0.16 ; the results are shown in figure 3.8. The direction of the flow is from top to bottom and each OSG has been annotated with a dashed line depicting the start of the recirculation zone and an image of the geometry to the left. The position of the recirculation zone is indicated by a drastic change in the streaklines. The well defined inlet streaks suddenly stop at the beginning of the zone where the oil-streak mixture is not significantly moved by the flow. The oil-streak results show that the OSG-2 recirculation zone pushes the furthest upstream, followed by the the OSG-3 zone, and finally the OSG-1 zone starts furthest downstream. 

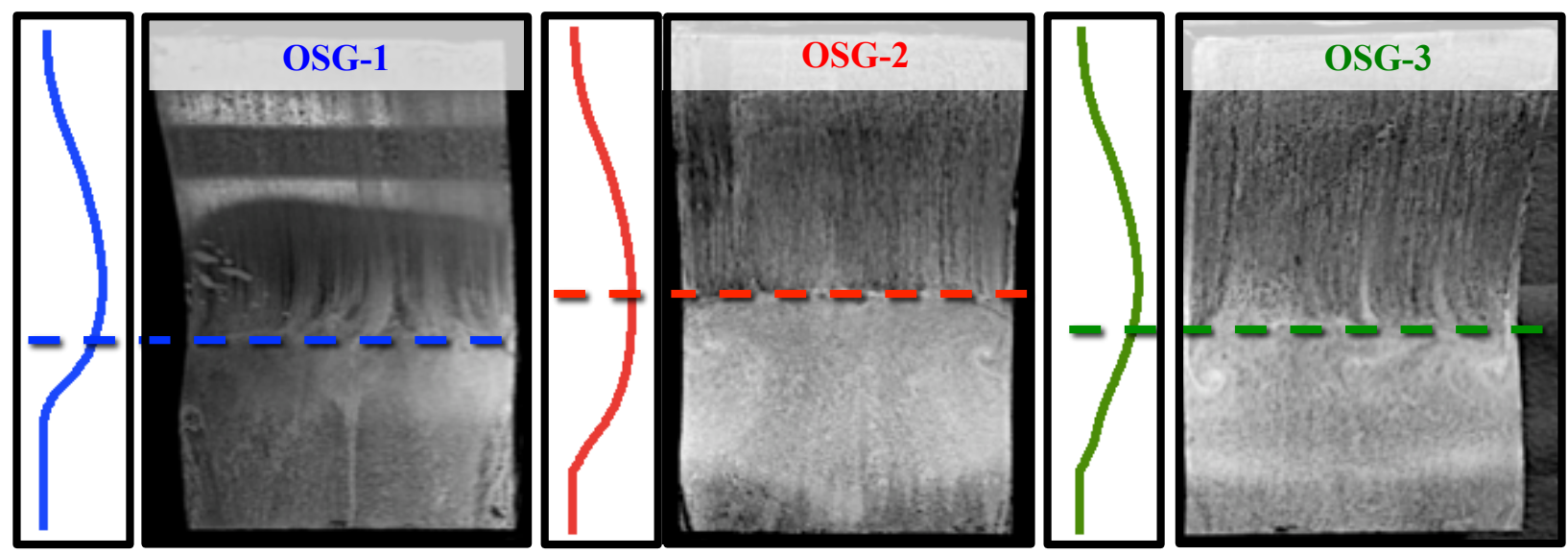

Figure 3.8: Oil streak images of each OSG at $\beta=0.16$ comparing recirculation zone size

\subsection{Efficiency Results}

Results for all three OSG flow paths using A4 Coarse Test Dust are shown in figure 3.9a. The core flow was held constant at $1.7 \mathrm{lbm} / \mathrm{sec}$ and three target scavenge splits of $\beta=0.14,0.16$, and 0.20 were used as primary test conditions. Additional tests for OSG-1 were conducted at lower scavenge splits to explore the low end operability of the IPS. The measured efficiencies fall with the expected range of values given by Filippone and Bojdo $\left.{ }^{[10]}\right)$. It is evident that the efficiency has a dependence on the scavenge split for all three OSGs. As $\beta$ is increased, the efficiency of the system is also increased; this implies that there are significant fluid effects on the particles within the IPS.

By averaging the data at each condition, the trends can be seen more easily in figure 3.9b. The efficiency data are in good agreement with previous IPS studies that were done, specifically Vittal et al. ${ }^{[4]}$ and Duffy ${ }^{[2]}$, This data validates the use of a rectangular geometry to study the multiphase interactions within the IPS and that the conditions are similar to a typical IPS system.

Relating this data to the oil-streak data, it is evident that there is a correlation between 


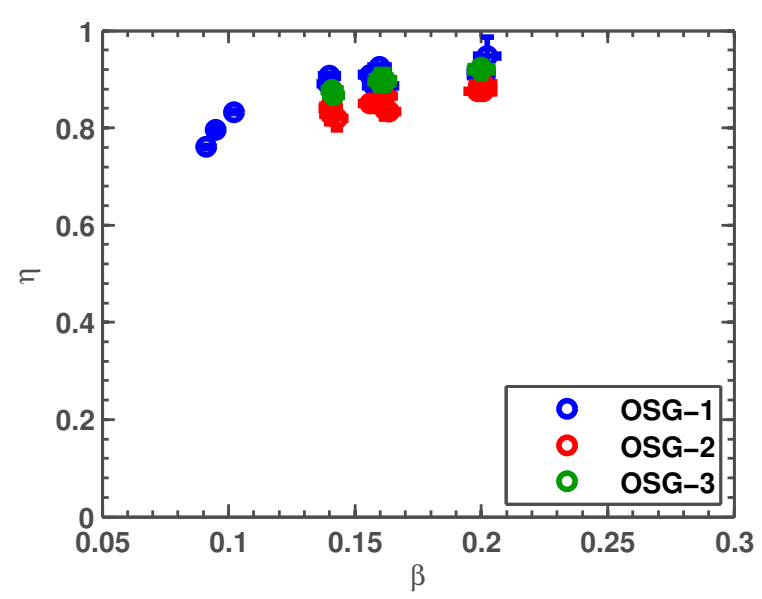

(a) Raw data

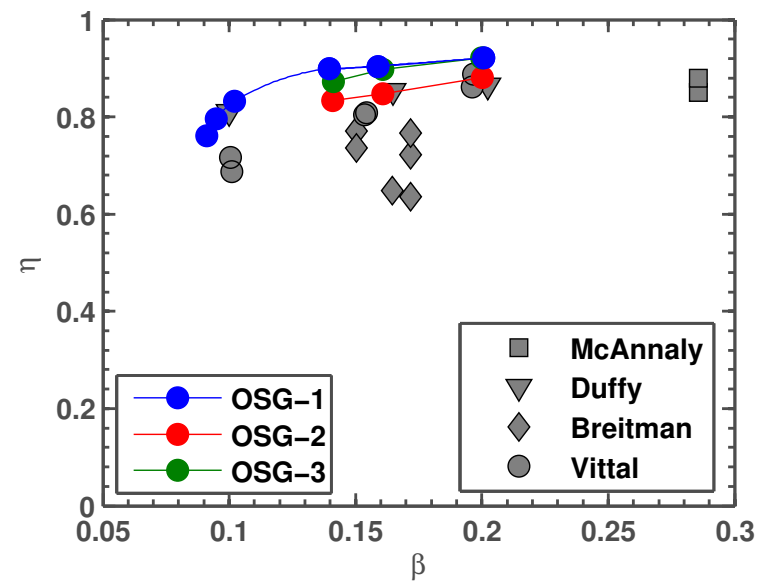

(b) Averaged data comparison

Figure 3.9: IPS Efficiency for A4 Coarse Test Dust

recirculation zone position and separation efficiency. The recirculation zone present for OSG-1 is positioned furthest downstream and has highest efficiency, while the recirculation zone in OSG-2 is positioned furthest upstream and has the lowest efficiency.

\subsection{Conclusions}

There is a significant correlation between the position of the recirculation zone and the efficiency of the IPS geometry. This finding supports the hypothesis that the recirculation itself has a significant impact on separation efficiency. The separation efficiency of an IPS is also dependent on the scavenge flow split; increasing the amount of scavenge flow results in an improvement in efficiency and as the scavenge flow split for OSG-1 approaches 0.10 there is a significant increase in separation performance sensitivity to $\beta$. Further work is planned to study the influence of the recirculation zone, including the use of mono-disperse spherical particles to eliminate variables concerning particle size and shape, and particle image velocimetry to study the flow field. 


\section{CHAPTER 4}

\section{Glass Sphere Efficiency}

\subsection{Introduction}

An Inertial Particle Separator (IPS) is a device installed on an aircraft to minimize the dust and debris ingested by the engine. These particles can be highly damaging to the internal components of a gas turbine engine, significantly reducing its lifetime ${ }^{[8-11,16]}$. Aircraft in sandy areas, particularly helicopters and other vertical takeoff and landing aircraft, are at enhanced risk because their downwash introduces particles into the air around the aircraft ${ }^{[27]}$. Of the current Engine Air Particle Separators (EAPS), IPS systems are ideal for their lower weight and pressure loss characteristics. However, they generally have the lowest separation efficiency when compared with Vortex Tube Separators (VTS) ${ }^{[28]}$ and Inlet Barrier Filters $(\mathrm{IBF})^{[10]}$. There would be a clear advantage if the efficiency of IPS systems could be improved to match that of VTS and IBF systems while maintaining pressure loss and weight savings. To make these improvements in IPS separation efficiency, a fundamental understanding of the particle sensitivity to flow characteristics is required. 


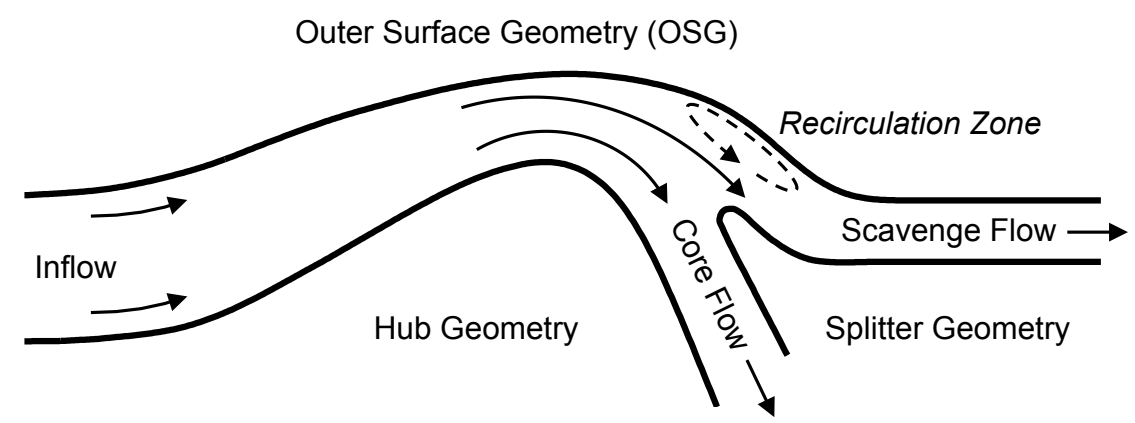

Figure 4.1: IPS nomenclature and flow features

The basic components of an IPS are shown in figure 4.1, and follow the same principles of a cyclone separator ${ }^{[29,30]}$. Contaminated air enters the inlet and the mixed flow makes a turn aft of the hub where the particles have sufficient inertia to prevent them from following the air around this sharp corner. Aft of the hub, the flow encounters the splitter where the particle-laden flow on the outside of the turn is scavenged away from the clean air nearer the hub. For particles that are too large to be significantly affected by the airflow, the IPS is designed such that the particles will bounce through into the scavenge flow path. The particle-laden scavenge flow path is then ejected from the aircraft while the cleaned air proceeds to the turbine engine.

Previously, efficiency measurements were made using graded test dusts such as ISO 12103-1, A4 Coarse Test Dust (also known as Arizona test dust or Arizona road dust). This dust has a broad range of particle sizes and shapes and has many components; primarily silica $\left(\mathrm{SiO}_{2}\right)$ and alumina $\left(\mathrm{Al}_{2} \mathrm{O}_{3}\right)$, and smaller amounts of other oxides of iron, sodium, calcium, magnesium, titanium, and potassium. The vast majority of previously published results use this standard or similar ranges of dust. Figure 4.2 shows previous measurements made by McAnally ${ }^{[1]}$, Duffy ${ }^{[2]}$, Breitman et al. ${ }^{[3]}$, and Vittal et al. ${ }^{[4]}$. This includes data by Barone et al. ${ }^{[5]}$ showing that the design of the OSG near the bifurcation zone can play a significant role 


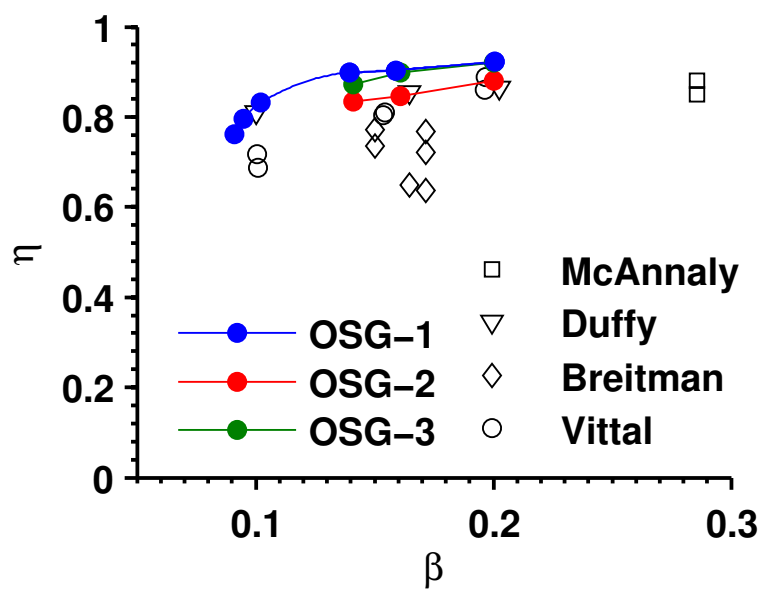

Figure 4.2: Previous studies of IPS Efficiency using A4 Coarse Test Dust ${ }^{[1-5]}$

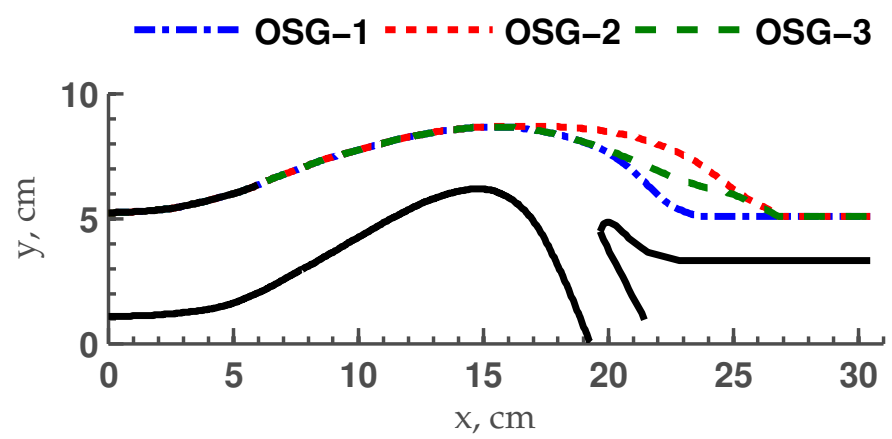

Figure 4.3: IPS test section geometries

in the efficiency of an IPS.

Figure 4.3 shows the coordinates for the three different OSGs used to study the effects of the recirculation zone. The OSG has significant impact on the size and location of the recirculation zone, resulting in a change in overall IPS efficiency. This effect on efficiency has been qualified using a test dust with a broad range of sizes and shapes yet more specific information is needed to understand the connection between these phenomena.

Separation efficiency is known to be even more sensitive to particle size, because that impacts both the particle inertia and particle drag. Therefore, there is a critical need to 
understand the effects of particle size on efficiency. Moreover, multiphase physics are difficult to assess when the testing particles are non-spherical and of different materials, as this creates large variability with respect to particle-wall reflection and particle drag characteristics ${ }^{[31]}$.

The objective of this study is to investigate nearly monodisperse particle sets of different sizes in a fundamental IPS geometry. Spherical particles with a single material component (glass) are used to allow a more fundamental characterization of the multiphase fluid dynamics. With this knowledge a model can be created to elucidate the particle dynamics occurring in an IPS.

\subsection{Methodology}

\subsubsection{IPS Airflow and Geometry}

A wind tunnel was designed to simulate the conditions that are present in an IPS, to explore the fluid dynamics present in the system. The tunnel utilizes a full scale, 2-D geometry that represents the flow through a quarter annulus of a typical large helicopter engine. When testing the IPS at full scale conditions, no special scaling requirements are needed to select special test particulate or other parameters.

IPS operation requires that air be removed from the flow through the scavenge leg of the system. There are two critical parameters regarding operation: the fraction of air used by the IPS to scavenge the particles and the mass separation efficiency of the IPS. The former can be described by the flow split, $\beta$, defined as the ratio of the mass flow of air entering the scavenge relative to the total mass flow of air through the system:

$$
\beta \equiv \frac{\dot{m}_{s}}{\dot{m}_{t}}=\frac{\dot{m}_{s}}{\dot{m}_{s}+\dot{m}_{c}}
$$


Because the mass flow rate entering the IPS is not directly measured, the total mass flow is determined by adding the scavenge and core flow rates. Fine tuning of the flow through the tunnel is obtained using two centrifugal blowers with independently controlled variable frequency drives. This allows the core mass flow to be set at a constant $1.71 \mathrm{lbm} / \mathrm{s}$ and the scavenge flow to be varied, achieving the desired $\beta$. The values are continually monitored through a data acquisition system and proper mass flow is maintained using a closed loop PID controller.

Figure 4.4 shows the wind tunnel, which has been specifically designed to conduct these experiments. Air enters the tunnel though the upper intake, passing through a hex-cell flow straightener followed by a converging nozzle into the test section. The test section is composed of five components, the hub geometry, splitter geometry, the OSG and two windows (one on either side). The IPS geometry pieces are made of machined aluminum and the windows are optically clear acrylic. The flow leaves the test section through both the core and scavenge flow legs through a diffuser followed by a particle filter. From there, the air travels to the two centrifugal blowers (one for the core leg and one for the scavenge leg) outside of the room.

Table 4.1 displays the characteristic values for the test section. The characteristic domain $(D)$ was chosen to be the length of the test section. This length was chosen because particles entering the IPS will experience changing fluid effects throughout the entire test section (particles initially pass the outward turn, followed by the hub-side turn, and then encounter the recirculation zone and scavenge region). Due to the complexity of the IPS system there are other choices for characteristic lengths available for use, such as the throat height $(H)$, the length of the hub, or the recirculation zone size, but only one will be used here for consistency. The estimated mean flow of the entire IPS test section was chosen to be the characteristic velocity. 


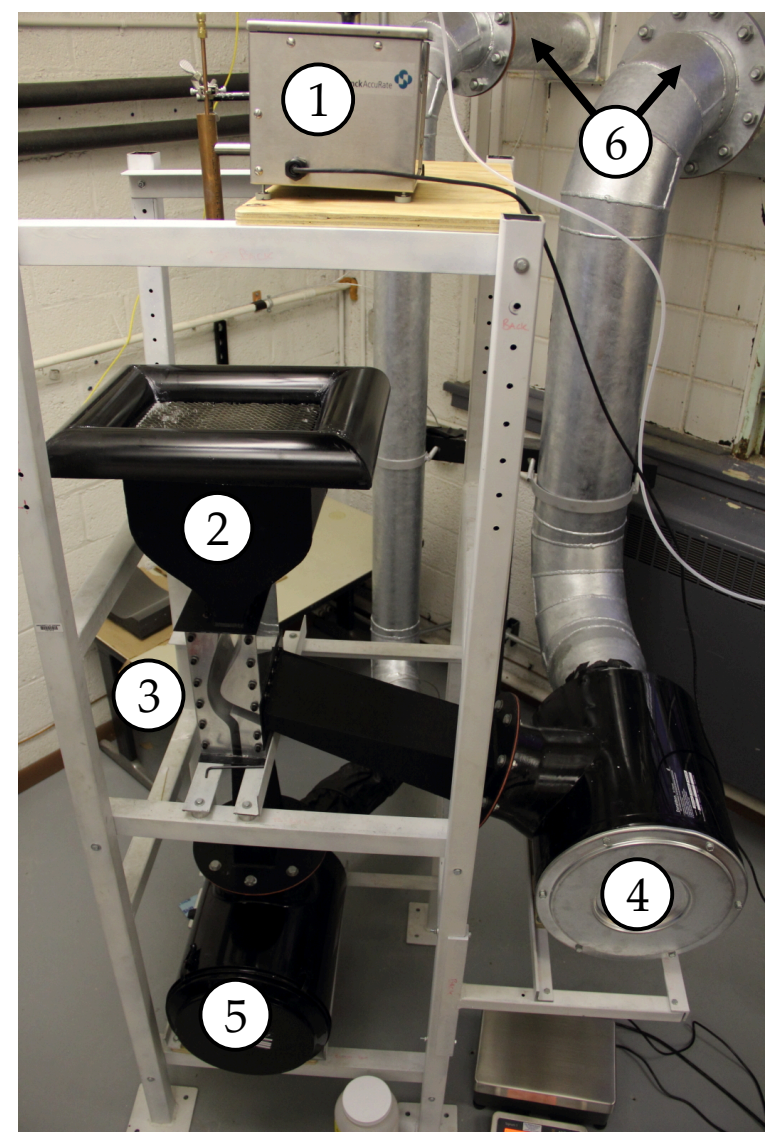

Figure 4.4: IPS Research Facility located at the University of Virginia showing: 1) the particle delivery system, 2) air intake, 3) test section, 4) core filter, 5) scavenge filter, and 6) facility air system

Table 4.1: Flow Characteristics

\begin{tabular}{|c|c|c|}
\hline Variable & \multicolumn{2}{|c|}{ Value } \\
\hline$\rho_{\text {air }}$ & 1.185 & $\mathrm{~kg} / \mathrm{m}^{3}$ \\
\hline$\mu_{\text {air }}$ & $1.926 \times 10^{-5}$ & $\mathrm{~kg} / \mathrm{m} \cdot \mathrm{s}$ \\
\hline$u_{D}$ & 93 & $\mathrm{~m} / \mathrm{s}$ \\
\hline$D$ & 0.30 & $m$ \\
\hline$H$ & 0.025 & $m$ \\
\hline$\dot{m}_{c}$ & 0.78 & $\mathrm{~kg} / \mathrm{s}$ \\
\hline$R e_{D}$ & $1.6 \times 10^{6}$ & \\
\hline
\end{tabular}




\subsubsection{Particle Characteristics}

Particles used in the experiment were selected based on the following requirements:

1. Spherical Particles to remove shape effects

2. Range of Stokes numbers covering the range of A4 Test Dust

3. Density within the range of A4 Dust, $2270 \mathrm{~kg} / \mathrm{m}^{3}<\rho_{p}<5240 \mathrm{~kg} / \mathrm{m}^{3}$ (A4 Average $=$ $\left.2880 \mathrm{~kg} / \mathrm{m}^{3}\right)$

The use of spherical particles eliminates the uncertainty of the shape factor and, more importantly, minimizes the dispersion created by nonuniform particle bouncing.

ȦngströmSphere Soda-Lime Beads, distributed by Fiber Optic Center, Inc. were selected based on availability in terms of relatively monodisperse distributions at a reasonable cost. In addition, these glass spheres are made of silica, the primary component of the test dust, and can be made into near perfect spheres. Three sizes were selected for testing, with nominal means of $10 \mu \mathrm{m}, 35 \mu \mathrm{m}$, and $120 \mu \mathrm{m}$ and density of $2.5 \mathrm{~kg} / \mathrm{m}^{3}$. The particles represent the distribution of A4 test dust with $35 \mu \mathrm{m}$ representing the mean and the $10 \mu \mathrm{m}$ and $120 \mu \mathrm{m}$ representing the smaller and larger sizes respectively.

The glass-spheres were analyzed to ensure their size and shape. Figure 4.5 shows images of each sphere size. The images show the particles to be spherical, but there seemed to be a size distribution that was broader than expected. To assess this, particles were analyzed by Powder Technologies, Inc. with results in figure 4.8 .

The distributions of the $10 \mu \mathrm{m}$ and $120 \mu \mathrm{m}$ particles are fairly monodisperse, but the $35 \mu \mathrm{m}$ particles have a broader distribution. These results are still very useful as they provide an approximation for the bulk of A4 test dust without shape and density variation. 


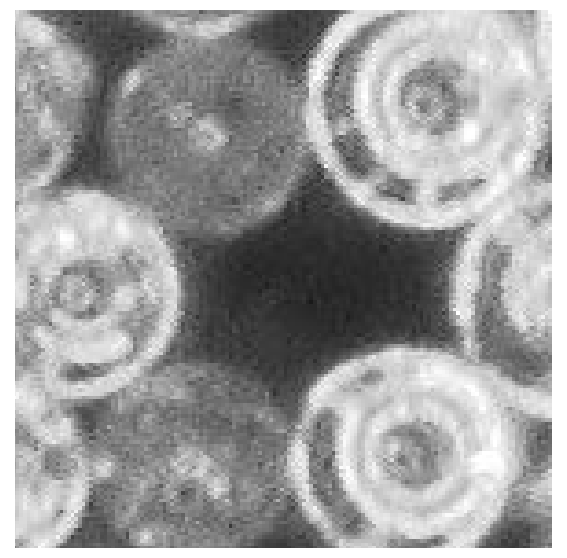

(a)

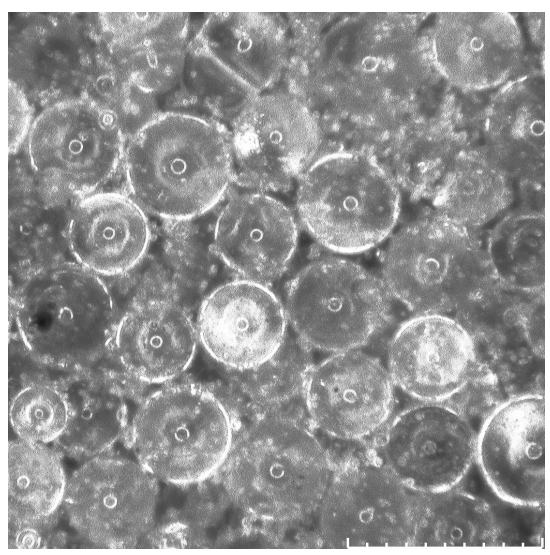

(b)

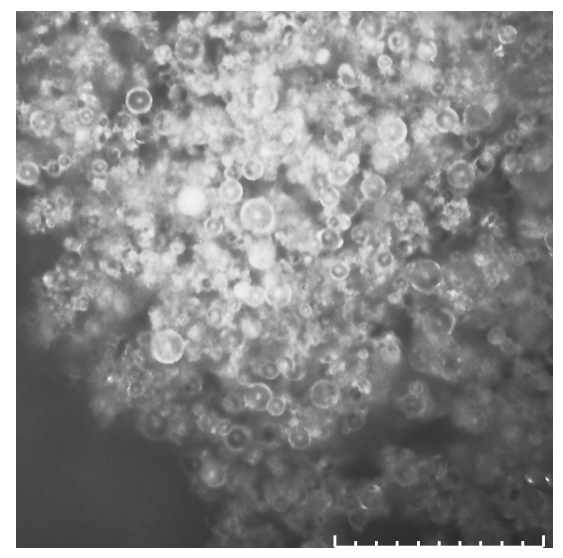

(c)

Figure 4.5: Microscope images of glass-spheres for the three nominal diameters: a) $120 \mu \mathrm{m}$, b) 35 $\mu m$ and c) $10 \mu m$ (note that images are at differing magnifications)

\subsubsection{Separation Efficiency Measurements}

Efficiency measurements were conducted at the University of Virginia using the IPS research facility. A detailed description of the facility and its operation can be found in Barone et al. ${ }^{[8]}$. Data were collected for each size particle at mass flow splits, $\beta$, of $0.14,0.16$ and 0.20. The tunnel was brought up to condition and approximately $0.1 \mathrm{~kg}$ of glass spheres were delivered into the inlet flow. Efficiency measurements were made by measuring the amount of particulate collected in particle filters located on the core and scavenge flow paths downstream of the test section.

The mass separation efficiency of an IPS is defined as the ratio of particulate mass entering the scavenge leg to the total mass of particulate entering the inlet of the IPS, Eq. (4.2).

$$
\eta \equiv \frac{m_{s}}{m_{t}}=\frac{\Delta m_{s}}{\Delta m_{s}+\Delta m_{c}}
$$

The particulate mass through the system is defined by the change in mass of the filters designed to catch the particulate in the core and scavenge flow legs. The total mass delivered to 


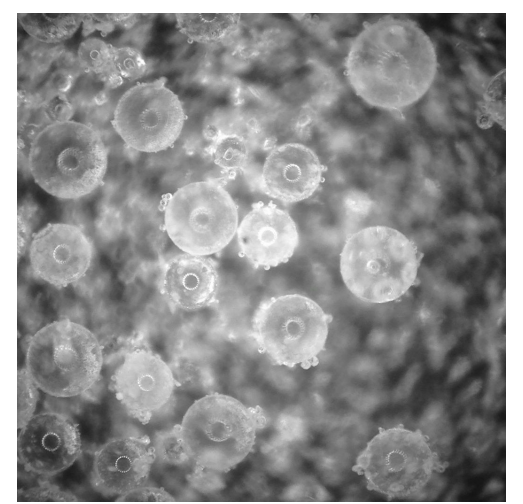

Figure 4.6: Microscope images of glass-spheres from scavenge filter

the system can also be measured using the particle delivery system, this measurement is used in the efficiency uncertainty calculation. The amount of particulate used in the experiment was selected to minimize the amount needed while maintaining small measurement error, calculated using Eq. (4.3)

$$
\delta \eta=\sqrt{\left(\frac{\Delta m_{s}}{\left(\Delta m_{c}+\Delta m_{s}\right)^{2}}\right)^{2}\left(\delta m_{r}{ }^{2}+\delta m_{b}{ }^{2}\right)+\left(\frac{\Delta m_{c}}{\left(\Delta m_{c}+\Delta m_{s}\right)^{2}}\right)^{2}\left(\delta m_{r}{ }^{2}+\delta m_{b}{ }^{2}\right)}
$$

where the residual mass $\delta m_{r}$ is the amount of mass lost between the particle delivery system and the air filters (typically 1-5g) and the balance error, $\delta m_{b}$ is the accuracy of the scale (about $0.08 g$ ). Thus, the net separation uncertainty was thus estimated to be around 1-2\%.

To ensure that efficiency measurements were not being affected by particle breakup (resulting in non-spherical particles), images were obtained of particles collected from the scavenge filter after several tests were run. Figure 4.6 is an image from an optical microscope showing particles of all sizes from the filter. There was no evidence of broken particles in the samples examined. 


\subsubsection{Particle Stokes Number}

The multiphase interactions in a particle laden flow can be very complex and several factors need to be taken into account when selecting particles for testing. The Stokes Number provides a tool to compare these factors. It is a non-dimensional parameter used to determine how a particle will react to the fluid flow field; this number is determined by simplifying the parameters of the flow.

The Domain Stokes Number is the ratio of the particle response time, $\tau_{P}$, and the domain fluid response $\tau_{D}$ time is the time it takes the fluid to move across the domain. The particle's response time is determined by its ratio of inertia to drag, $m v / F_{d r a g}$, and the fluid response time is the how long it takes a fluid element to traverse the domain, $D / u_{D}$. The ratio of these two time constant results in the domain Stokes Number, Eqn. (4.4):

$$
S t_{D} \equiv \frac{\tau_{P}}{\tau_{D}}=\frac{\rho_{p} d_{p}{ }^{2}}{18 \mu_{\text {air }} f} \frac{u_{D}}{D}
$$

A particle with a Stokes number much less than 1 will track the fluid flow because the particle's response time is shorter than the fluid's response time. Conversely, particles with values much greater than 1 will be effected only slightly by the flow, resulting in the trajectories of these particles being dominated by bouncing on the walls. Particles with Stokes numbers on the order of 1 will have a balance of inertial forces and fluid forces, the trajectory of these particles are more difficult to predict and are thus of the most interest.

Table 4.1 displays values used for the Stokes analysis. The particle density is based on the density of silica, $\mathrm{SiO}_{4}$, which is the primary component of the A4 test dust and soda-lime glass. The Stokes correction factor, $f$, is assumed to be 1 for spherical particles because the shape effects of the test dust are unknown. The domain is the length of the IPS test section, and the domain velocity is the inlet velocity of the test section. 


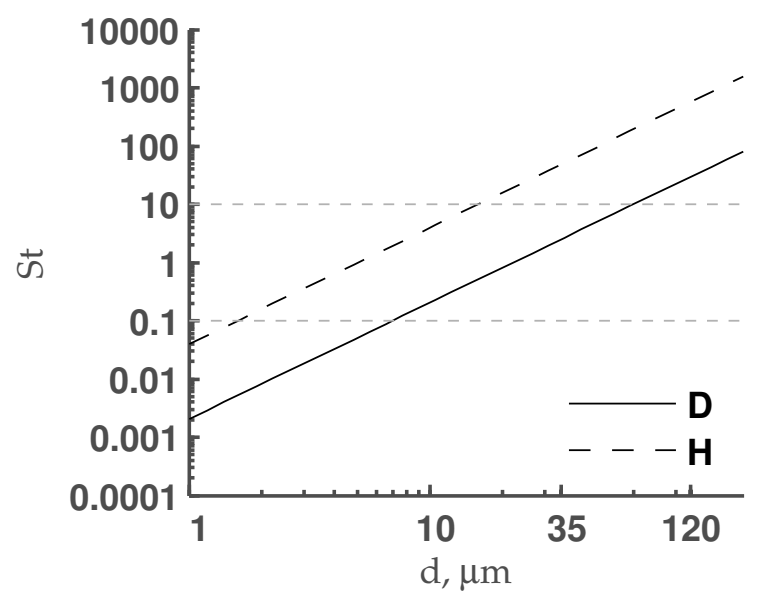

Figure 4.7: Stokes numbers based on domain length (D) and throat height $(\mathrm{H})$ as a function of particle size

Figure 4.7 shows the domain Stokes number as a function of particle diameter where $D=0.3 m$ and $u_{D}=93 \mathrm{~m} / \mathrm{s}$. It should be noted that $S t_{D}$ is expected to capture particle trajectories associated with inviscid turning effects of the air flow streamlines. However, effects associated with flow separation and unsteadiness would be best described by using a flow time scale that is based on the integral turbulent time-scale, $\tau_{\Lambda}$, instead of the domain time-scale $\left(\tau_{D}\right)$ of Eqn. (4.4). However, such analysis is difficult because the bifurcated flow has been shown to have a highly complex turbulent flow field ${ }^{[32]}$, so that $\tau_{\Lambda}$ is expected to vary widely throughout the flow. However, one may estimate that these effects are substantially influenced by the flow separation length. Based on measurements of the velocity field by Barone et al. ${ }^{[32]}$, the flow separation length varies with flow split and geometry but is which is approximately proportional to the flow path height at the throat, $H$. The height-based Stokes number $\left(S t_{H}=\tau_{p} \cdot u_{H} / H\right)$, is also included in figure 4.8 based on the height of the throat at $x \approx 15 \mathrm{~cm}$ where $H=2.5 \mathrm{~cm}$ and $u_{H} \approx 137 \mathrm{~m} / \mathrm{s}$. Since $S t_{H}$ is greater than $S t_{D}$ for a given particle diameter, one may expect that that the largest particles will be more influenced by inviscid flow turning than by turbulent flow structures. 


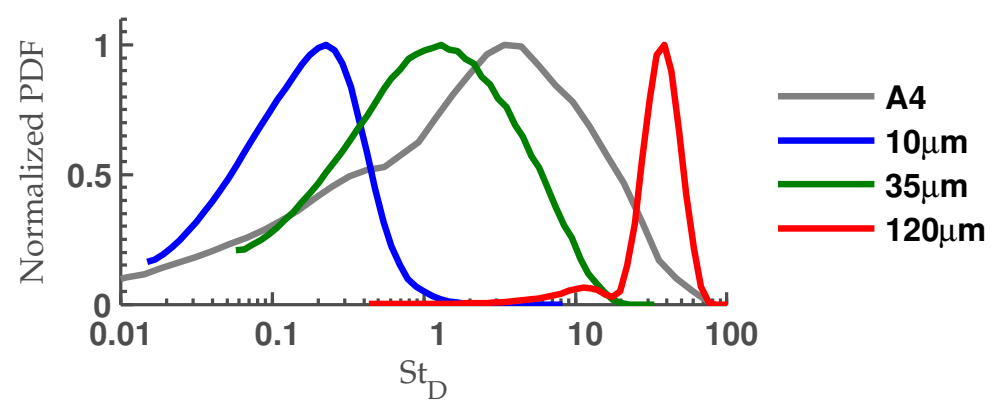

Figure 4.8: Measured size distribution of glass-spheres

Figure 4.8 shows the variation in domain Stokes number as a function of particle diameter (from Eqn. (4.4)). Because the Stokes number has a linear relation to diameter on a logarithmic scale, the same curve can be plotted to either diameter or Stokes number; in this case any point on the plot can be referenced to the lower abscissa as diameter and to the upper abscissa is the stokes number. This plot shows that the A4 test dust spans a very large range of Stokes numbers with many particles much less than 1 and much greater than 1 with the majority of particles falling in the critical range between 0.1 and 10 , where trajectories become difficult to predict. Additionally the three sizes of glass spheres fall into interesting categories of $S t_{D}<1, S t_{D} \sim 1$, and $S t_{D} \gg 1$, this should provide valuable insight into particles with varying levels of drag influence and bounce-dominated particles. If one assumes that the turbulent-scale influences are approximately represented by $S t_{H}$, this indicates that these effects will be weak for the two largest glass beads (for which $S t_{H} \ll 1$ ), but may be significant for the smallest glass beads (for which $S t_{H} \sim 1$ ).

\subsubsection{Separation Efficiency Model}

Using data provided by multiple sets of particles with differing diameters, it should be possible to model the separation efficiency as a function of diameter. While the IPS has a very complex flow structure, it can be compared to a simpler problem with a known solution. Specifically, 


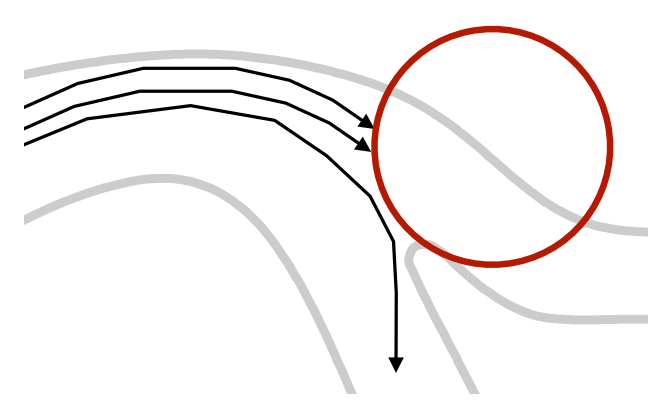

Figure 4.9: Adaptation of the model from Langmuir and Blodgett ${ }^{[6]}$ to the IPS geometry

the IPS is similar to particulate flow impacting a cylinder. The scavenge flow path can be thought of as half the cylinder and the core flow path as the air around the cylinder as shown in figure 4.9 .

Previous work on the impact of particles on cylinders is summarized and re-evaluated using computational methods by Finstad et al. ${ }^{[33]}$ based largely on the work by Langmuir and Blodgett ${ }^{[6]}$. The impact efficiency of droplets of various sizes (based on Stokes number) are calculated by integrating the equations of motion for water droplets in a steady potential flow around an infinitely long cylinder. These calculations result in a simple equation:

$$
\eta=\frac{S t_{D}}{S t_{D}+C}
$$

This equation has further been modified by several authors with various values of the constant, $C$, the Stokes number calculation, or the form of the equation using empirical data. Similarly, the equation can be modified for use in an IPS and a value for $\mathrm{C}$ can be determined though an empirical fit. One significant modification to the equation is required: the particles in the IPS will have a minimum efficiency greater than zero because the very small tracer particles will follow the flow into the scavenge flow path. Thus, the minimum separation efficiency will be equal to the flow split. Taking this into account, Eqn. 4.5 can be rewritten in the following form: 


$$
\eta=(1-\beta) \frac{S t_{D}}{S t_{D}+C}+\beta
$$

The constant $C$ can be calculated using the known particle distribution and efficiency for A4 test dust. By integrating Eq. 4.5 over the entire range of particle sizes weighted by their mass percentage, an estimated efficiency for the dust can be determined. The value of $C$ can then be varied until the efficiency of the model and the actual measured efficiency are equal.

\subsection{Results}

The efficiency of the three OSGs with the glass particles is shown in figure 4.10. Each OSG has near $100 \%$ efficiency for the $120 \mu m$ particles. Incorporating this fact with the knowledge that IPS geometries are designed to bounce inertially dominated particles $\left(S t_{D} \gg 1\right)$ into the scavenge leg of the diffuser, it can be inferred that these large particles are bouncing through the IPS with minimal effect from the fluid forces. 


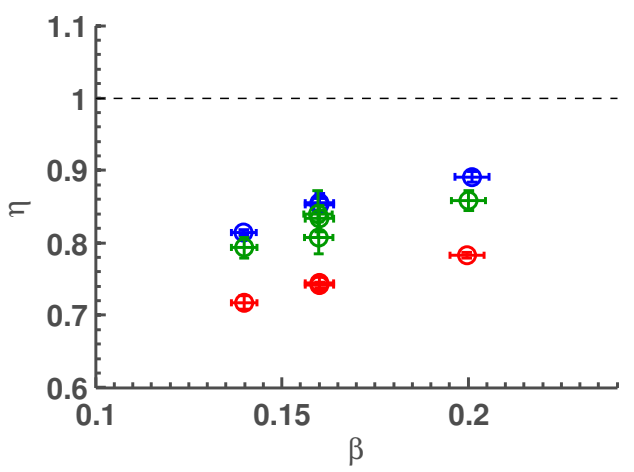

(a)

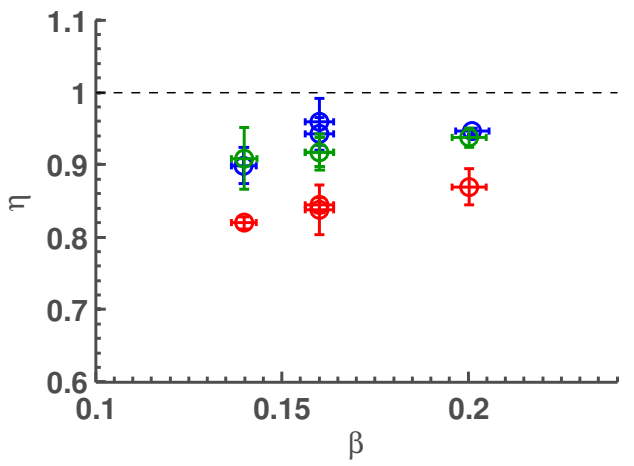

(b)

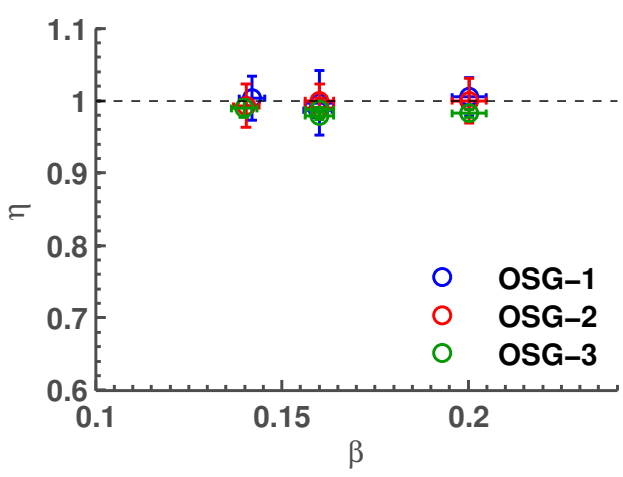

(c)

Figure 4.10: Glass bead efficiency as a function of flow split for different diameters a) $10 \mu m$, b) $35 \mu \mathrm{m}$ and c) $120 \mu \mathrm{m}$ 
With the smaller $35 \mu \mathrm{m}$ particles, the separation efficiency is reduced for each OSG and further reduced with the smallest $10 \mu \mathrm{m}$ particles. This shows that as the particle Stokes number is reduced the fluid forces are influencing the separation efficiency more. Looking at the overall performance of the glass sphere separation efficiency, OSG-1 performs the best followed by OSG-3 then OSG-2; this is consistent with the results found using the A4 test dust from Barone et al. ${ }^{[5]}$.

The cause of this difference was postulated by Barone et al. ${ }^{[5]}$ who demonstrated that the location of the recirculation zone (furthest upstream for OSG-2, and furthest downstream for OSG-1) was correlated with the separation efficiency of the OSG. Because the IPS has the same mass flow of air through the core and scavenge flow legs (regardless of OSG), it is reasonable to assume that the fluid dynamic forces causing the change in separation efficiency are related to the OSG and the influence it has on the flow field at the entrance to the scavenge flow leg.

Figure 4.11 displays the data reorganized as a function of particle diameter for the baseline flow split of 0.16. As with A4 test dust, particle separation efficiency increases with an increase in the flow split for the $10 \mu \mathrm{m}$ and $35 \mu \mathrm{m}$ particles, but the $120 \mu \mathrm{m}$ particles are unaffected, confirming that the large particles are dominated by inertia.

The constant for Eqn. (4.6) was obtained using the efficiency data and size distribution for A4 test dust as described in the previous section. At a flow split of 0.16 , A4 dust has an efficiency of 0.90 (figure 4.2). Using the Stokes analysis and distribution from figure 4.8, the corresponding value of $C$ is 0.046 . With the constant value computed for this case, Eqn. (4.6) can be applied as an inviscid model to the distributions for the glass spheres as well. Figure 4.12 displays the efficiency of the OSG-1 at $\beta=0.16$; the glass sphere efficiencies are also shown, as well as the width of the line represents the range of Stokes numbers in which $95 \%$ of the particles fall into. It appears that the model predicts the separation efficiency as 


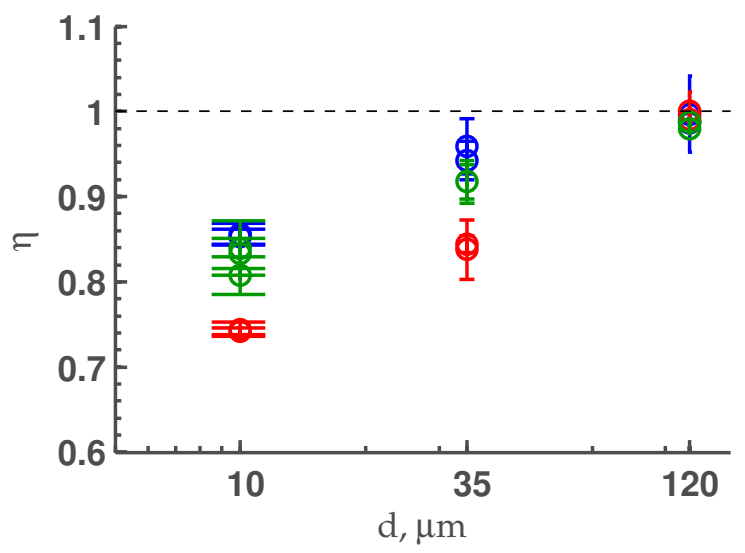

Figure 4.11: Glass bead efficiency as a function of diameter with a flow split of $\beta=0.16$

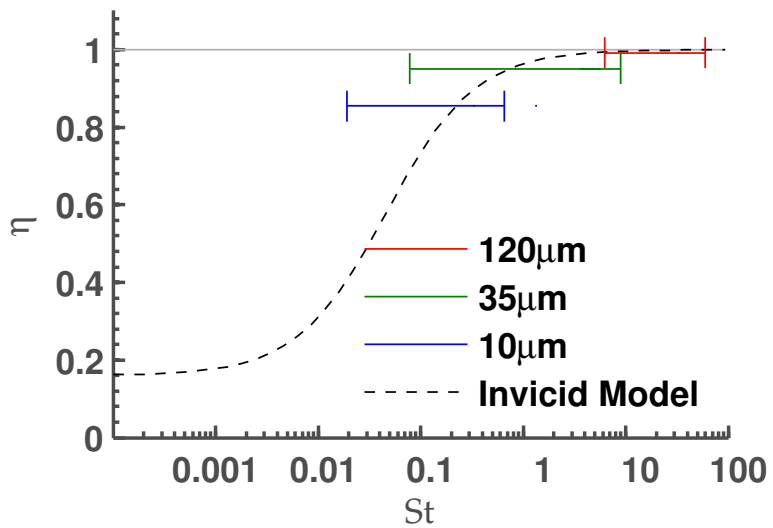

Figure 4.12: Efficiency predictions based on previous A4 experiential data

a function of Stokes number.

With verification of the model, it is then applied to each of the particle sets. Figure 4.13 displays the comparison between predicted efficiencies to the actual measured efficiencies for each OSG. The prediction and measured values of efficiency will always match because the model is tuned to A4 test dust.

The prediction works very well for the $120 \mu \mathrm{m}$ and $35 \mu \mathrm{m}$ particles, but significantly 


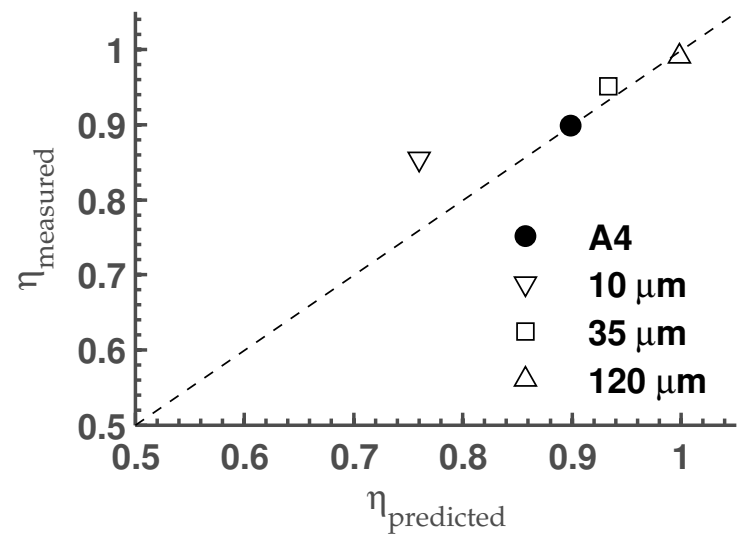

(a)

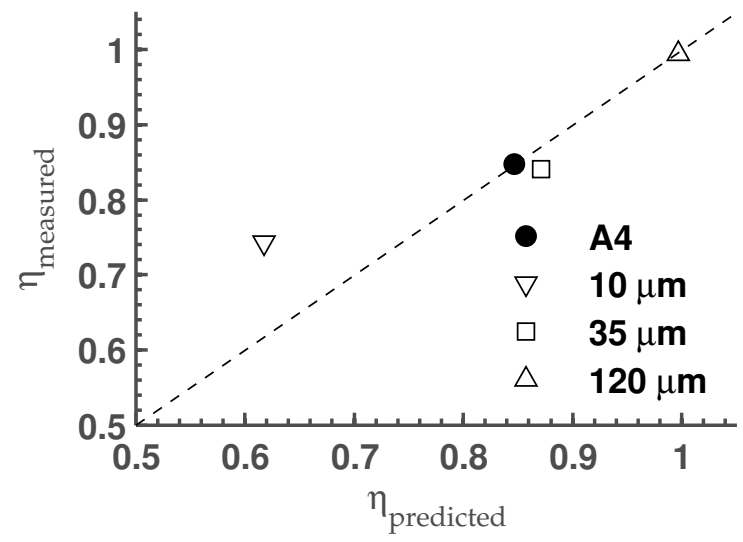

(b)

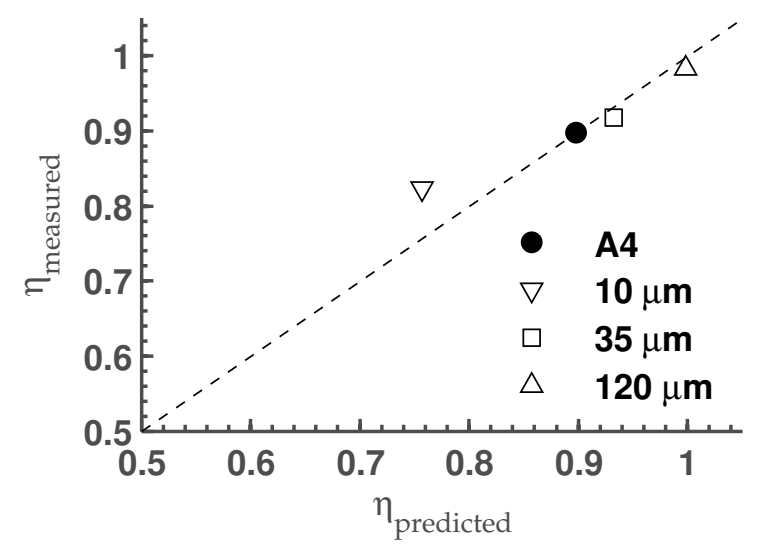

(c)

Figure 4.13: Efficiency prediction comparison for $\beta=0.16$ showing a) OSG-1 $(C=0.046)$, b) OSG-2 $(C=0.12)$, and c) OSG-3 $(C=0.051)$

over-predicts the efficiency of the $10 \mu \mathrm{m}$ particles in each case.* The discrepancy between the model and the data can be attributed to the complicated interactions that occur in the IPS. The model is based on a simple steady flow around a cylinder, where the IPS geometry is much more complicated and includes the presence of a flow feature like the recirculation zone. As discussed in Sect. 4.2.4, effects of of flow separation and turbulence are expected to be important for the smallest size particles, and this is consistent with the inviscid model of

${ }^{*}$ The data in figures 4.13 and 4.14 were previously reported by Barone et al. ${ }^{[34]}$ with the abscissa and ordinate accidentally axis switched. 
Eqn. (4.6) yielding good agreement for only the largest particles. Considering this fact with the knowledge that there is an effect on efficiency caused by the OSG geometry leads to the conclusion that internal flow structures affect particles with Stokes numbers on the order of 1 and less. This is an important step in identifying the mechanisms involved in IPS efficiency.

Additionally, the equation can be applied to varying values of $\beta$. Figure 4.14 displays three different values of $\beta$ for OSG-1. In these cases the value for $C$ was held constant at 0.046, derived from the baseline flow split for the IPS. As evidenced in these displayed figures, the equation does a sufficient job predicting separation efficiency for changing flow split values. The difference in the $10 \mu \mathrm{m}$ particles is still seen in the $\beta=0.16$ and $\beta=0.20$ cases, but is not as pronounced in the $\beta=0.14$ case.

Furthering this method, it is possible to compare the model to an example from the literature. Vittal et al. ${ }^{[4]}$ provided experimental data for an IPS using A4 test dust. Hamed et al. ${ }^{[7]}$ utilized the same geometry in a combination of CFD and Lagrangian particle computations to study the separation efficiency of discretely sized particles. Vittal et al. measured a separation efficiency of $88.8 \%$ with $\beta=0.20$ and Hamed et al. computed a separation efficiency ${ }^{\dagger}$ of $94.8 \%$ with $\beta=0.24$.

As shown previously in figure 4.14, the inviscid model does well in comparing the same geometry over a range of $\beta$; therefore, it is possible to compare the separation efficiency from each study. Using the experimental A4 data from Vittal et al. and solving the inviscid model equation, $C=0.063$. Applying the value from Hamed et al. of $\beta=0.24$, the inviscid model predicts $\eta=89.4 \%$. Thus according to the inviscid model, the CFD is over predicting the separation efficiency by $5.4 \%$.

Figure 4.15 demonstrates the difference in efficiency between the inviscid model and

\footnotetext{
${ }^{\dagger}$ Hamed et al. do not provide a published separation efficiency for A4 test dust. It is calculated here using their separation efficiency for discretely sized particles and the distribution of A4 test dust from figure 4.8 .
} 


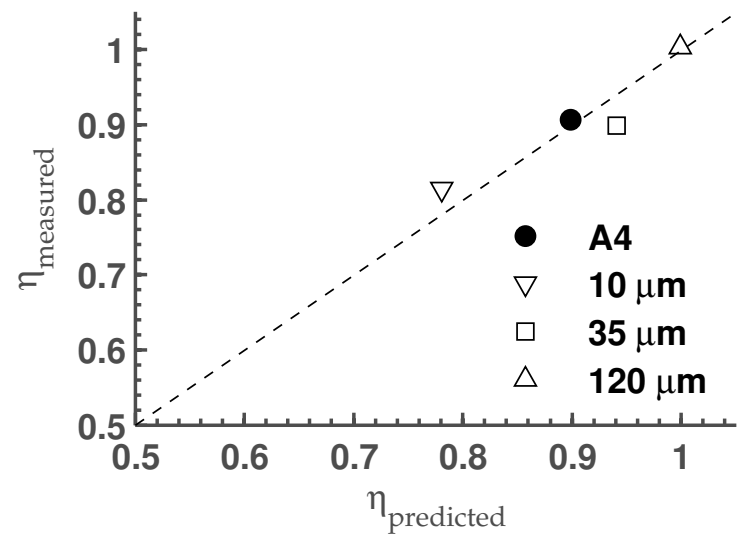

(a)

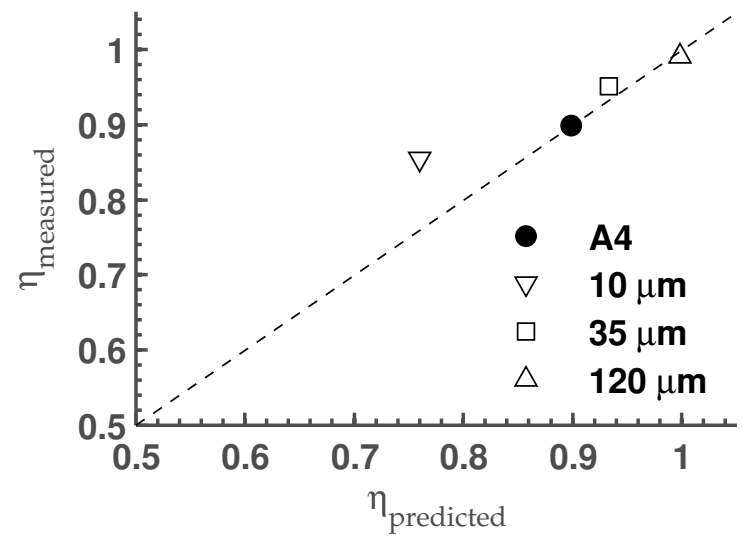

(b)

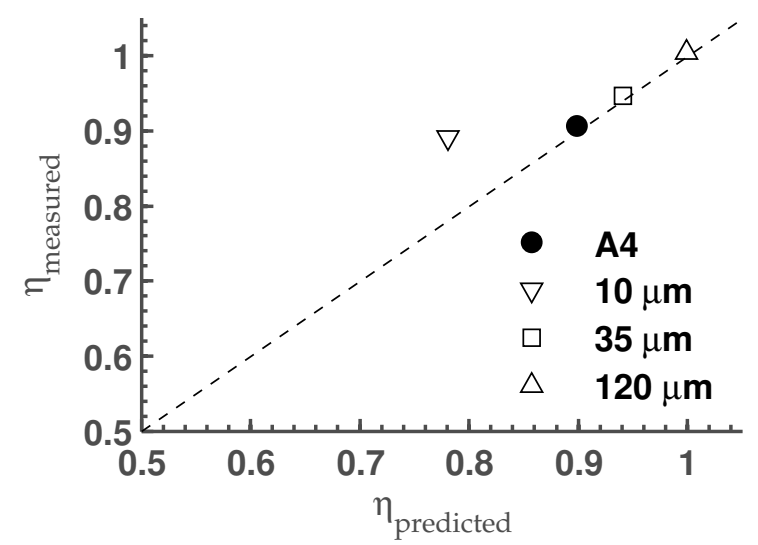

Figure 4.14: Efficiency prediction comparison $(C=0.04)$ for OSG-1 showing a) $\beta=0.14$, b) $\beta=0.16$, and c) $\beta=0.20$

the CFD for particles ranging in size from $1 \mu \mathrm{m}$ to $100 \mu \mathrm{m}$. The inviscid model matches the calculations well for $S t<0.05$ and $S t>5$; however, the inviscid model predicts much lower separation efficiencies for particles within the range $0.05<S t<5$, corresponding to particle diameters of $5 \mu \mathrm{m}$ to $20 \mu \mathrm{m}$. This discrepancy is in the range of Stokes numbers where particles are especially susceptible to variations in fluid forces. These variations could be due to large scale turbulence that is not captured in the averaged flow calculations used by Hamed et al. 


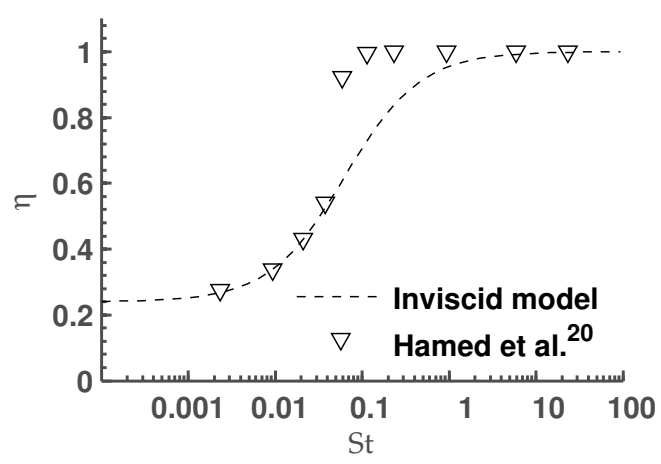

(a)

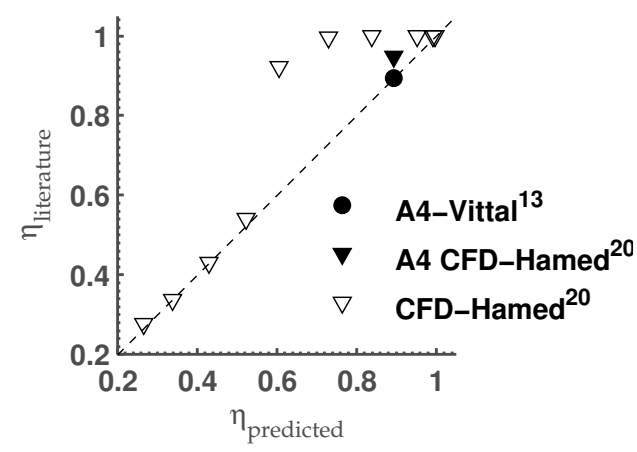

(b)

Figure 4.15: Efficiency prediction model applied to the data from Vittal et al ${ }^{[4]}$ and Hamed et al. ${ }^{[7]}$

\subsection{Conclusions}

Efficiency calculations were completed using uniform particles to isolate effects on IPS efficiency. Three different particle sizes were tested at three different flow splits on each geometry. Using the test results, a model was developed to predict the separation efficiency of particles based on their stokes number. Large particles were shown to have near $100 \%$ efficiency at any flow split, leading to the conclusion that they bounce through the IPS as designed. As particles become smaller, their separation efficiency decreases due to the increase of fluid influences until the particles are so small that their trajectories are completely dominated by fluid forces.

Particles with large Stokes numbers and very small Stokes numbers follow the predictive model well; however, particles with stokes numbers near 1 show significant discrepancies. Because of the balance of fluid and inertial forces these particles it is reasonable to assume that there are significant fluid forces affecting the particles that cannot be predicted using a steady or averaged flow solution. Further work is needed to identify these phenomena and how they can be overcome to increase IPS efficiency. 


\section{CHAPTER 5}

\section{IPS Fluid Dynamics}

\subsection{Introduction}

Inertial Particle Separators (IPS) are installed on an aircraft to minimize the dust and debris ingested by the engine. These ingested particles can be highly damaging to the

internal components of a gas turbine engine, significantly reducing its lifetime ${ }^{[8-11,16]}$. Aircraft operating at low altitude in sandy areas are at particular risk. This is especially true for helicopters as their rotor-downwash lifts particles off the ground and entraining them in the surrounding air. In the extreme conditions of brownout, the particle concentrations can reach $2.5 \mathrm{~g} / \mathrm{m}^{3}$ obscuring vision and causing a high mass flux of particles to be injected into the engine inlets ${ }^{[11]}$.

IPS systems are generally axisymmetric and can be either outward-turning or inwardturning. The basic flow components of an outward-turning IPS are shown in Fig. 4.1. Contaminated air enters the inlet and the mixed flow (particles and gas) makes an initial turn away from the hub and then the core flow it turned back towards the hub while a scavenge 
flow undergoes a less dramatic turn. For an inward-turning IPS, the flow path is similar except that turns are reversed so that the flow initially turns towards the hub and then away from it. In either case, the successive turns are ideally designed so that particles have sufficient inertia to prevent them from following the air into the core flow. Ideally, the overall geometry allows all the particles to be scavenged so that the core flow is completely clear of particles, i.e. has clean air. The particle laden flow in the scavenge path is then ejected from the aircraft while the cleaned air proceeds to the turbine engine.

IPS systems are generally designed to have high separation efficiency (fraction of particle removed from airstream entering engine) combined with minimum losses in pressure ${ }^{[10]}$. To meet both objectives, the flow path must be carefully designed in terms of the amount and location of turning as well as the geometry of the flow bifurcation. The splitter geometry (which separates the core and scavenge flows) is key to controlling the flow and to directing the particle paths towards the scavenge flow. The Outer Surface Geometry (OSG) and the hubgeometry primarily dictate the degree of flow turning needed to achieve this particle scavenging. The influence of the OSG can be especially important as it can govern the boundary layer separation of the recirculation zone that starts along its surface at a streamwise position just upstream of the splitter (see Fig. 4.1). The presence of this region is due to the high degree of diffusion of the flow, resulting from a scavenge passage sized to give adequate particle capture. Understanding how the flow reacts to these turns and the bifurcation of the flow path is critical to furthering IPS design.

Unfortunately, there are no published experimental results for an IPS flow that describe the velocity field. To the author's knowledge, the only experimental flow feature data currently available are surface flow oil-streak measurements by Barone et al. ${ }^{[5]}$, and this study only examined the influence of OSG geometry on the location and size of the recirculation zone. Despite this lack of experimental data, there have been several computational studies ${ }^{[7,14-20]}$ 
Table 5.1: Summary of results from Barone et al. for efficiency and recirculation zone location by OSG

\begin{tabular}{ccccc}
\hline OSG & $\eta$ & $x_{\text {start }}$ & $x_{\text {end }}$ & $L$ \\
\hline 1 & 0.90 & $19.2 \mathrm{~cm}$ & $23.2 \mathrm{~cm}$ & $4 \mathrm{~cm}$ \\
2 & 0.89 & $16.5 \mathrm{~cm}$ & $26.8 \mathrm{~cm}$ & $10.3 \mathrm{~cm}$ \\
3 & 0.85 & $18.9 \mathrm{~cm}$ & $26.0 \mathrm{~cm}$ & $7.1 \mathrm{~cm}$ \\
\hline
\end{tabular}

indicating the high degree of interest in the IPS flow fields. However, these computational studies primarily focused on the average flow field and not the unsteady dynamic flow structures that are present in a recirculation zone. Therefore, the turbulent flow characteristics of this class of IPS flows have not been measured and are largely unknown.

It is clear that experimental data is needed to better understand the mean and unsteady flow dynamics of an IPS system and it's effect on the particle trajectory ${ }^{[20]}$. Obtaining and analyzing such data is the objective of the present study. To allow the investigation to be fundamental and highly relevant, the experiments have carried out with well-characterized two-dimensional flow path designed for full-scale particle and flow conditions. The velocity fields are obtained optically using Particle Image Velocimetry (PIV) in the test section where the flow turns inward and bifurcates. This technique is capable of determine the entire two-dimensional velocity field without interrupting the flow. High-speed video of glass spheres is taken to qualify time-resolved flow structures to supplement the quantitative velocity fields obtained with PIV. To the author's knowledge, this is the first time any such data has been taken in an IPS and the results discussed herein are expected to be useful in validating particle separator design tools. 


\subsection{Methodology}

\subsubsection{Facility}

The experiments are conducted in a wind tunnel, located at the University of Virginia, that is designed to reproduce the full-scale gas flow path velocities and length-scales, as well as the full-scale particle sizes present in an IPS. To simplify the introduction of particulate into the flow field, the wind tunnel is designed vertically so the airflow and particles are primarily directed downward. Previous computational studies have shown the gravitational effects within the IPS to be negligible ${ }^{[20]}$. The IPS test section utilizes a two-dimensional geometry that represents the flow through a quarter annulus of a typical large helicopter engine. The aspect ratio of the flow path is large enough to avoid the influence of end-wall effects at the center $50 \%$ of the span. The two-dimensional representation of an IPS with acrylic sidewalls allows for optical access to the entire flow path. The geometry also eliminates the radial variations associated with inward-turning or outward-turning axisymmetric geometries, representing a fundamentally average flow path of those two axisymmetric cases. The test section used in this study can be seen in Fig. 5.1 (with the flow oriented in the downward direction) and captures the salient geometrical and flow features of Fig. 4.1 (where flow is in the right-ward direction). The particle separation efficiency by mass has been previously quantified in detail using A4 test dust ${ }^{[5]}$ and several sizes of glass spheres ${ }^{[34]}$.

The IPS concept requires that air be removed from the flow through the scavenge leg of the system. The flow split, $\beta$, is defined as the ratio of the mass flow rate of air entering the scavenge leg relative to the total mass flow rate of air through the system. The total mass flow rate through the tunnel was fine-tuned using two centrifugal blowers for the scavenge and core flow legs. Each blower was independently controlled with a variable frequency drive 


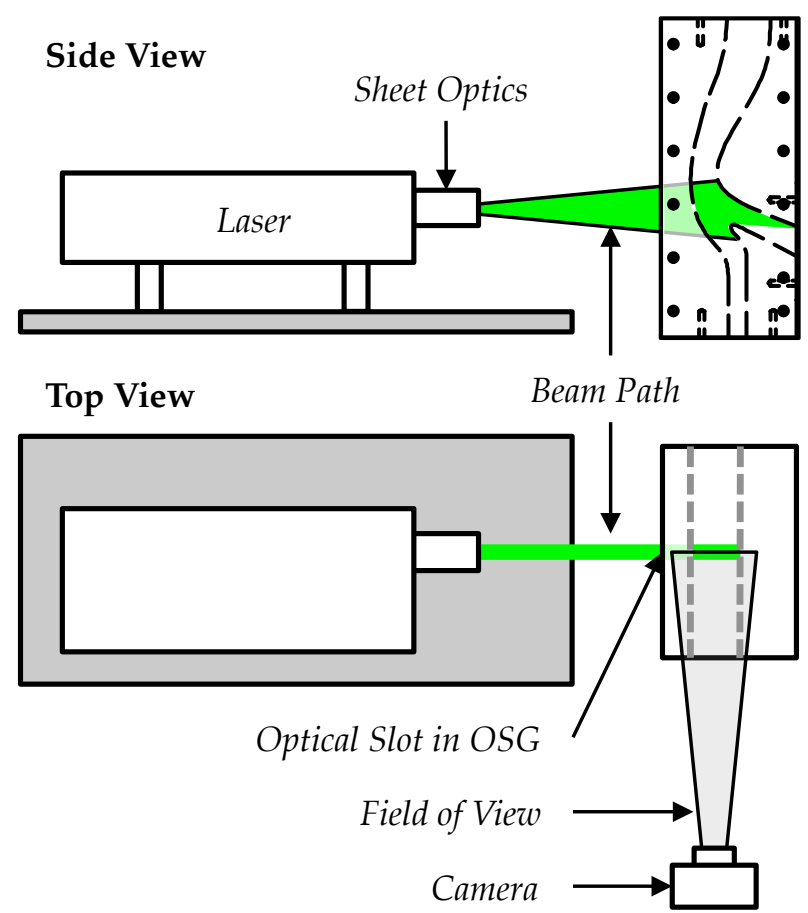

(a)

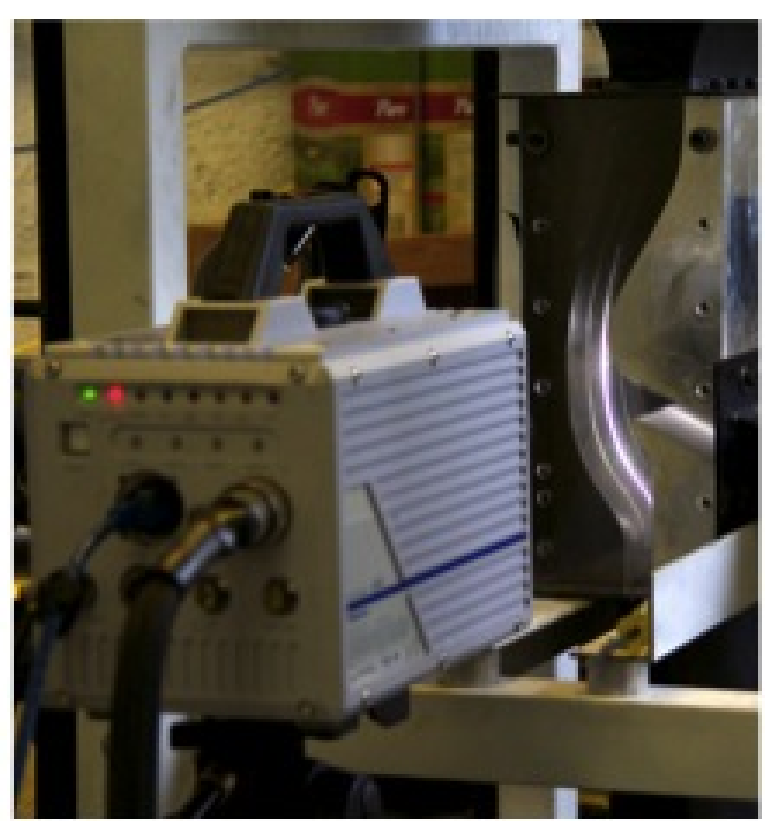

(b)

Figure 5.1: Experimental setup showing a) schematic of the PIV setup and b) a picture of the high speed video setup

Table 5.2: Test conditions for Gas PIV

\begin{tabular}{ccccccc}
\hline OSG & Seed & $\beta$ & $T$ & $R H$ & $p$ & $\mathrm{~N}$ \\
\hline 1 & Olive Oil & 0.16 & $75.0 \mathrm{~F}$ & $45 \%$ & $29.9 \mathrm{inHg}$ & 2000 \\
2 & Olive Oil & 0.16 & $65.2 \mathrm{~F}$ & $34 \%$ & $29.7 \mathrm{inHg}$ & 1000 \\
3 & Olive Oil & 0.16 & $68.3 \mathrm{~F}$ & $54 \%$ & $30.2 \mathrm{inHg}$ & 1000 \\
\hline
\end{tabular}

with flow rate measured by Tek-Air VT-2000 flow meters. For all conditions in this study, the core mass flow is fixed at a constant $1.71 \mathrm{lbm} / \mathrm{s}$ and the scavenge flow was set at $0.326 \mathrm{lbm} / \mathrm{s}$. These mass flow rates yield the baseline flow split value of 0.16 which is within the range of typical IPS operation; the details of the facility operation are documented by Barone et al. ${ }^{[8]}$.

To conduct the PIV and the high-speed video measurements, the test section OSG was 
modified to allow access for a laser sheet. The OSG was machined to include a centerline vertical slit $(0.32 \mathrm{~cm}$ wide and $17.8 \mathrm{~cm}$ long) for illumination of the scavenge flow leg, bifurcation region, and the hub-side turn. In addition, four other slits, $3.2 \mathrm{~cm}$ and $6.4 \mathrm{~cm}$ off-center in both directions, were machined for possible future experiments. The slits were sealed along the flow surface of the OSG using optically clear tape (McMaster-Carr PN: 7562A21) that is $0.09 \mathrm{~mm}$ thick, including the silicon adhesive. A particle separation efficiency test was conducted using the modified OSG-1 piece at the baseline 0.16 flow split and there was no difference to the efficiency reported by Barone et al ${ }^{[5]}$, within the experimental error of $1-2 \%$.

\subsubsection{Particle Image Velocimetry of Gas Velocity}

PIV is a method commonly used in gas flows to quantify the velocity field. It employs tracer particles seeded into the flow that are small enough in size so that they faithfully follow the surrounding airflow. These particles are illuminated, normally with a laser sheet, and tracked using a camera. The flow's velocity field can be calculated using a pair of images that contain the same set of particles after they have travelled a short distance. The field is subdivided into a discrete grid of sub-regions referred to as interrogation spots to obtain a two-dimensional matrix of velocity vectors. For each of these sub-regions, the particles that have traversed a small distance across two successive images are cross-correlated. The distance vector from the cross-correlation is divided by the time between the two images resulting in the velocity vector. By taking a large number of these image pairs throughout an experiment, an average flow field can be determined, along with the statistical variations present.

PIV measurements were conducted done using the hardware specified in Tbl. 5.3. The 
Table 5.3: PIV System

\begin{tabular}{lc}
\hline Laser & \\
\hline Manufacturer & New Wave \\
Model & Nd:Yag Solo PIV III \\
Pulse Duration & $3-5 \mathrm{~ns}$ \\
Pulse Frequency & $7 \mathrm{~Hz}$ \\
Pulse Energy & $150 \mathrm{~mJ}$ \\
$\Delta t$ & $2 \mu \mathrm{s}$ \\
\hline Camera & \\
\hline Manufacturer & TSI \\
Model & PowerView Plus \\
Resolution & $2048 \times 2048$ \\
Lens & Nikon $120 \mathrm{~mm}$ with $1.4 \mathrm{x}$ adapter \\
\hline Processing & TSI Insight 4G \\
\hline Software & Minimum Intensity Subtraction \\
Background & Two Image Cross Correlation \\
Method & Recursive Nyquist \\
Grid & $64 \times 64$ \\
Initial Spot Size & $48 \times 48$ \\
Final Spot Size &
\end{tabular}

flow was seeded using olive oil droplets generated using a TSI Oil Droplet Generator (Model 9307-6) that generates mean droplet diameters of $1 \mu \mathrm{m}$. This oil drop diameter has been shown to accurately capture both mean and turbulent dynamics of the air flow ${ }^{[35]}$. The droplets were delivered to the tunnel through a seed bar suspended above the tunnel connected by flexible PVC tubing. The seed bar is made of 3/4 inch diameter, Schedule 40 PVC pipe capped at one end with $0.32 \mathrm{~cm}$ holes drilled at $2.54 \mathrm{~cm}$ intervals. The seed bar is aligned with the center of the tunnel, which keeps the droplets away from the windows. Without the seed bar, olive oil droplets were found to coat the windows, causing them to fog up, which disrupts the camera's field of view. 


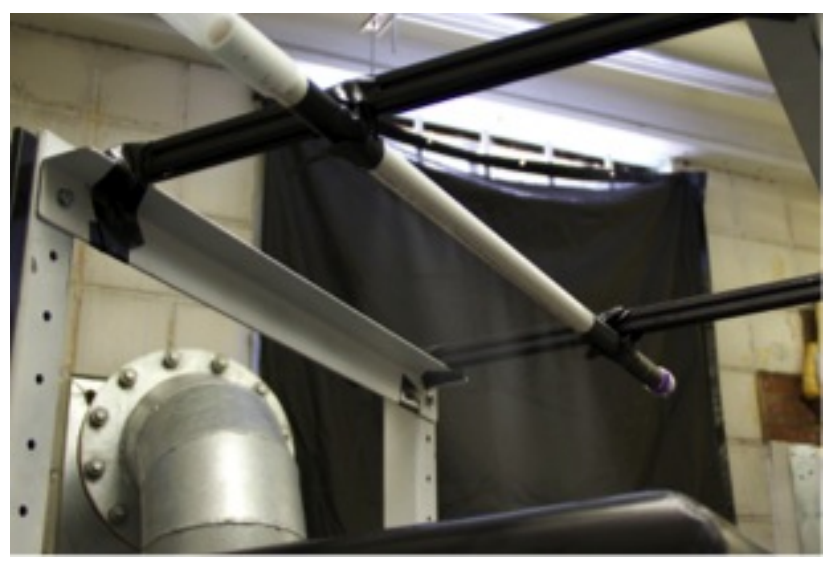

Figure 5.2: Olive-oil seed bar installed in the IPS research tunnel

Figure 5.1 shows a schematic of the PIV setup. The laser used to illuminate the particles in the flow field is a dual-head Nd:Yag laser from New Wave. This laser operates at 532nm and employs the two separate lasing cavities to produce two short, bright pulses $(<5 n s$, $150 \mathrm{~mJ})$ with a small time separation $(2 \mu \mathrm{s})$. These two pulses taken in rapid succession illuminate the particles that are captured by the camera to produce the image pair that is used to calculate the velocity field. The camera used is TSI PowerView plus CCD with a $2048 \times 2048$ resolution. The system is capable of capturing image pairs at a rate of $7 \mathrm{~Hz}$, which allows a large data set (e.g. 1000-2000 image pairs) to be taken within a few minutes of tunnel operation. However, this temporal resolution is not high enough to capture the time-correlated flow unsteadiness, as this would require speeds on the order of 20,000 $\mathrm{Hz}$. Therefore, high-speed video (discussed below) was used to supplement the PIV data to help understand the particle response to the dynamic structures present in the flow field.

The PIV images were post-processed using the software package Insight 4G from TSI. A background mask was calculated using the minimum intensity of each pixel over the entire image data set. This background image was then subtracted from each individual image to improve the signal. Processing was done using a Recursive Nyquist Grid with an initial 
coarse spot size of $64 \times 64$ pixels followed by a second pass with a $48 \times 48$ pixel spot size. The $2 \mu s$ separation between images in each pair was chosen so that particles would be displaced approximately $1 / 4$ of the interrogation zone spot size, maximizing the processing effectiveness. Post-processing of the data was then done to eliminate vectors with low signal to noise ratios. The resulting vector fields were then processed using MATLAB to obtain the important flow characteristics from the data.

\subsubsection{Data Convergence}

Because the IPS presents such a complex flow field, it is important to determine whether the average data reported are statistically converged. The sensitivity of the mean and standard deviation values were investigated as a function of how many vectors were required to produce the final value. To demonstrate, a point was selected at the edge of the recirculation zone $(x=19.2 \mathrm{~cm}, y=6.6 \mathrm{~cm})$ to quantify the statistical convergence properties of a sample velocity vector. From a total of 2000 image pairs, 1896 usable vectors were acquired at this location and the obtained mean velocity was $77 \mathrm{~m} / \mathrm{s}$ and the standard deviation was $14 \mathrm{~m} / \mathrm{s}$. These reference values $\left(\mu_{\text {ref }}\right.$ and $\left.\sigma_{r e f}\right)$ were used as an estimate of the statistically converged values. Figure 5.3a examines the mean and standard deviation normalized by the reference values at this point as a function of the number of vectors used in the calculation. It can be seen that the mean converges to within $5 \%$ of the reference value using only 32 vectors while the standard deviation converges to within $5 \%$ of the reference value using 428 vectors. Extending this analysis to every point in the field of view, Fig. 5.3b shows the number of vectors required for $5 \%$ convergence to the mean reference value. Most of the points converged within 150 vectors, but there are some points, notably near the OSG, that did not fully converge until nearly 1000 vectors were used. This slower convergence is attributed to high 
degree of velocity fluctuations relative to the mean flow since this region is characterized by a slow average flow field with high unsteadiness. In addition, the recirculation zone caused the olive oil to occasionally deposit on the wall. This accumulation of oil would produce bright spots in the images, hindering the quality of the vectors obtained in this area.

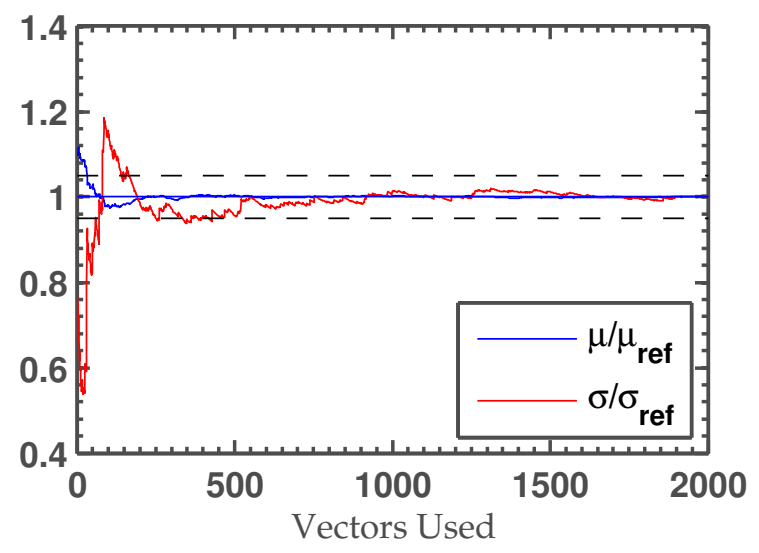

(a)

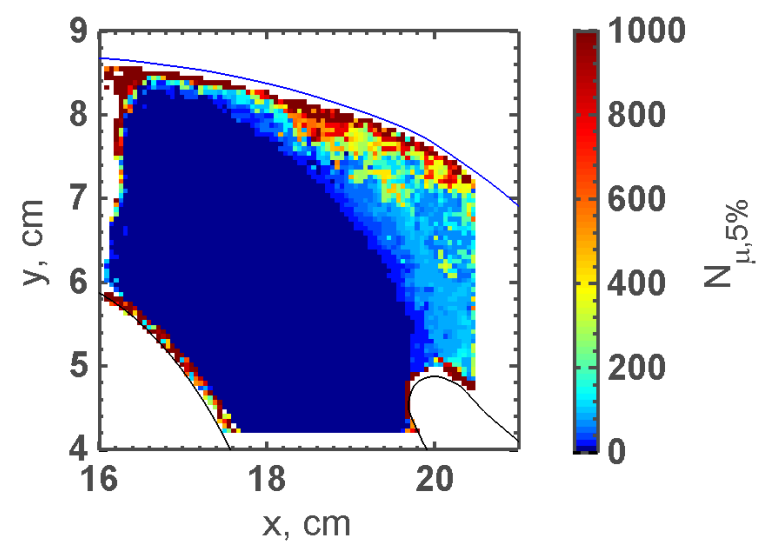

(b)

Figure 5.3: Data Convergence, showing a) Statistical convergence of velocity and turbulence data at $x=19.2 \mathrm{~cm}$ and $y=6.6 \mathrm{~cm}$ and b) number of vectors required to reach $5 \%$ convergence for the entire flow field.

\subsubsection{High-Speed Video of Glass Particle Dynamics}

High-speed video was used to capture the time-accurate particle dynamics of $10 \mathrm{um}$ glass spheres in the unsteady flow. A Photron SA4 camera was set up utilizing the same lens and field of view as the PIV camera (shown if Fig. 5.1) and took images at $20 \mathrm{kHz}$ to capture the flow dynamics. This camera has a pixel resolution of $512 \times 352$ and a shutter time of $50 \mu s$. The lighting was achieved without an expensive pulsed lasers source by delivering an intense light from a Fiber-Lite MI-150 through fiber optics that put the light directly up to the center slot in the OSG. The raw images from the camera were exported and processed using MATLAB, where they were inverted and level-adjusted to aid in visualization. 
The choice of glass spheres for the high-speed video was based on understanding the effects of flow unsteadiness on the fundamental particle dynamics, especially their intermittent and undesirable ingestion into the core stream. The specific particles chosen were characterized by a narrow distribution of particle sizes and were highly spherical to eliminate complexities associated with the particle shape effects. In addition, these particular sizes are critical as they represent the lower end of the particle size distribution of typical test dusts. The $10 \mu m$ particles were also found to have a large undesirable influence on separation performance as noted by Barone et al. ${ }^{[34]}$.

\section{$5.3 \quad$ Results}

\subsubsection{OSG-1}

Properties of the mean flow for the baseline case (OSG-1, $\beta=0.16$ ) are shown in Fig. 5.4. The inflow velocity on the (entering from the left) has a velocity gradient with higher speeds near the hub-side (lower y-values) where the majority of the flow is turning downward into the core flow leg as expected. A small fraction of the incoming flow is split off, at much lower speeds, into the scavenge flow leg (on the right). The splitting of the flow creates a very large velocity gradient between the high-speed core flow leg and lower-speed scavenge flow leg. Furthermore, at the top of the scavenge region there is significant region of near-zero velocity in fluid flow field, but no indication of reversed flow. This lack of a well-defined recirculation region in the average velocity magnitude plot was somewhat surprising as the wall-streak measurements of Barone et al. showed the existence of a recirculation zone on the OSG surface between $x=19.2 \mathrm{~cm}$ and $23.2 \mathrm{~cm}$. This incongruence of the PIV and the flow visualization results (with respect to flow separation) is particular to OSG-1, whereas 
OSG-2 and OSG-3 demonstrate recirculation in both the wall-streak visualization and in the mean PIV flow fields. The lack of mean reverse flow in the OSG-1 velocity field may be partly attributed to the inability to obtain velocity measurements close to the wall. However, instantaneous images of the velocity (as will be discussed later) indicate the existence of a highly intermittent recirculation zone for OSG-1, whereby it is sometimes fully attached and sometimes significantly separated. The OSG-1 recirculation thus occurs less often than those of OSG-2 and OSG-3, and is also smaller and less coherent when it does occur. As such, OSG-1 represents a flow with an adverse pressure gradient just beyond the conditions for mean incipient separation. In contrast, OSG-2 and OSG-3 represent conditions whereby the adverse pressure gradient is unquestionably strong enough to cause mean separation.

The standard deviation of the velocity magnitude for OSG-1 is shown in Fig. 5.4b and indicates a significant amount of deviation in the regions where the recirculation should be located according to the oil-streak measurements. This suggests that there is more happening in the instantaneous flow fields than is perceived in the average quantities. The span-wise vorticity (Fig. 5.4c) shows an area of high shear, indicative of the viscous effects associated with bifurcation of the incoming flow. Fig. 5.4d is the number of usable vectors obtained from the PIV measurements at each location. The PIV software is able to calculate vectors for nearly every image $(>90 \%)$ for most of the flow, especially to the portion entering the core flow. As such, there are more than enough vectors to compute the average flow field in these regions, as shown in the convergence plot. Notably, there is a loss of vectors the edge of the inflow due to the edge laser sheet (e.g. particles were not sufficiently illuminated). In addition, there is a drop-off in vector count near the walls due to surface reflections. Therefore, these regions may have some uncertainty with respect to the magnitude of the velocity fluctuations, but the vector count is large enough to accurately quantify the mean flow. There is a loss of vectors $(\approx 50 \%)$ along the OSG at $x=19.3 \mathrm{~cm}$. A build up of olive oil would occur at this 


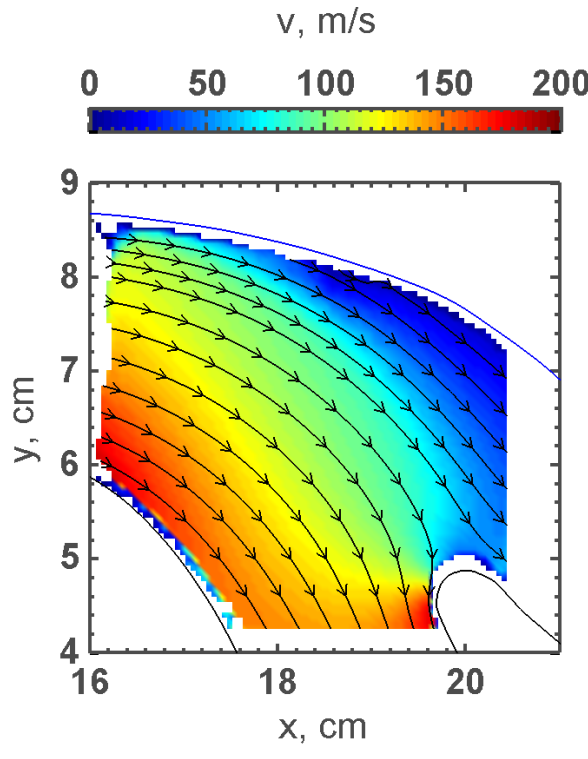

(a) Velocity Magnitude

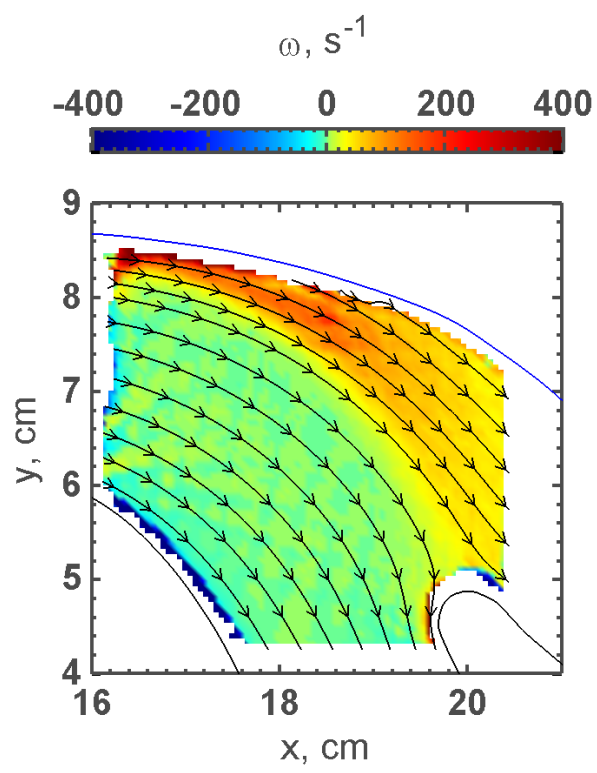

(c) Spanwise Vorticity

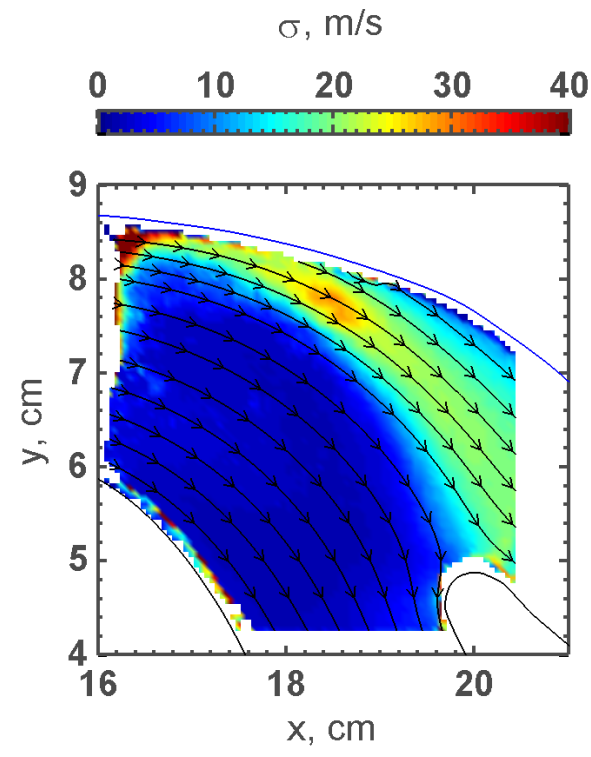

(b) Standard Deviation

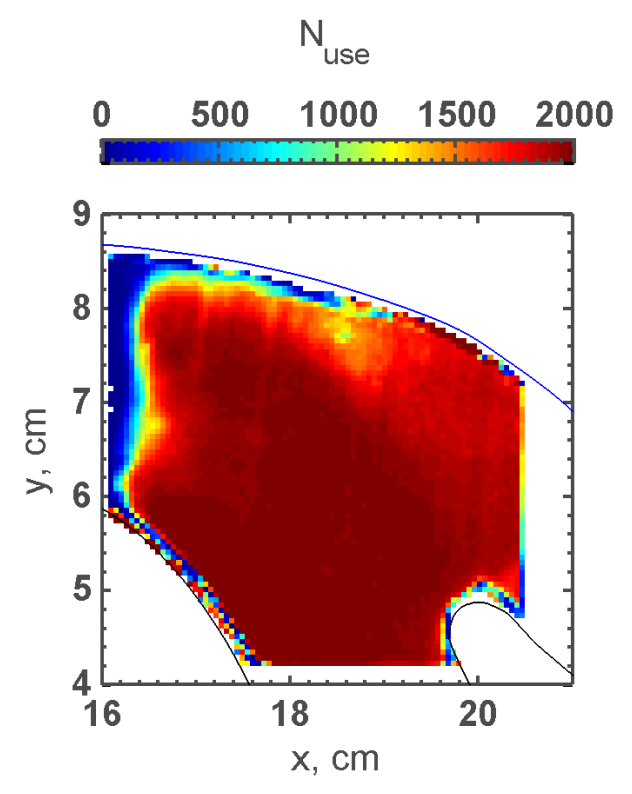

(d) Usable Vectors

Figure 5.4: Average quantities based on PIV measurements for OSG-1 at $\beta=0.16$ 
location, which produced a large reflection in turn over-saturating a small region of pixels in the camera. This location corresponds to the start of the recirculation zone as indicated by the previous oil-streak measurements.

In the bifurcation region documented by the PIV measurements, one may identify three critical planes of interest for the airflow flux as shown in Fig. 5.5d:

1. the incoming flow into the region at $x=17 \mathrm{~cm}$

2. the core flow exiting at $y=5.4 \mathrm{~cm}$

3. the scavenge flow exiting at $x=20 \mathrm{~cm}$

These three planes and the OSG form a two-dimensional control volume, which is useful in analyzing the spatial and temporal stability of the flow.

The spatial distribution of the mean velocity normal to the three flux surfaces is shown in Fig. 5.5a. Figures 5.5b, 5.5c, and 5.5d are plots the normal component of the mean velocity (solid lines) as well as the deviation bounds (dashed lines) for the incoming, core, and scavenge planes, respectively. The deviation bounds are defined by the mean velocity $+/$ one standard deviation. The incoming mean flow profile (Fig. 5.5b) has an average velocity of about $100 \mathrm{~m} / \mathrm{s}$ outside of the boundary layers, with a somewhat higher velocity near the hub. The accompanying deviation bounds have a relatively small spread about the mean. Such relatively weak velocity fluctuations indicate a nearly steady incoming flow, as is to be expected from the upstream contraction section designed for this facility. The core flow profile (Fig. 5.5c) shows nearly uniform flow velocities outside of the boundary layers and a small spread in the deviation bounds. The former indicates low spatial distortion and the latter indicates low unsteadiness, both of which are desirable properties for flow entering the engine performance. 


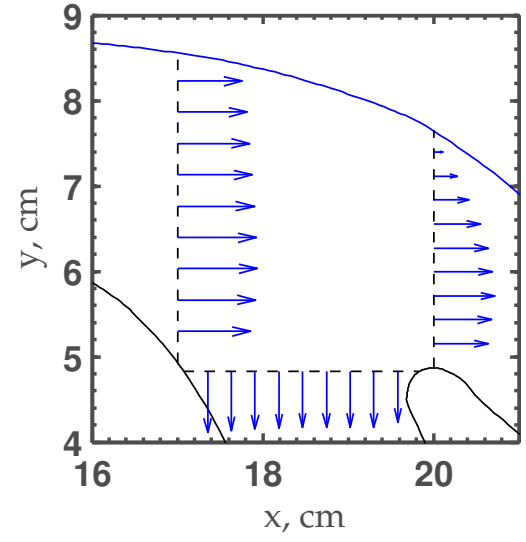

(a)

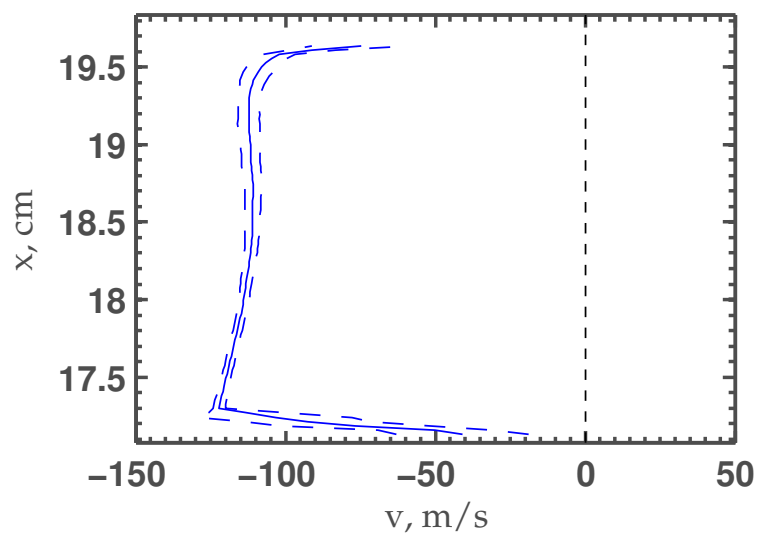

(c)

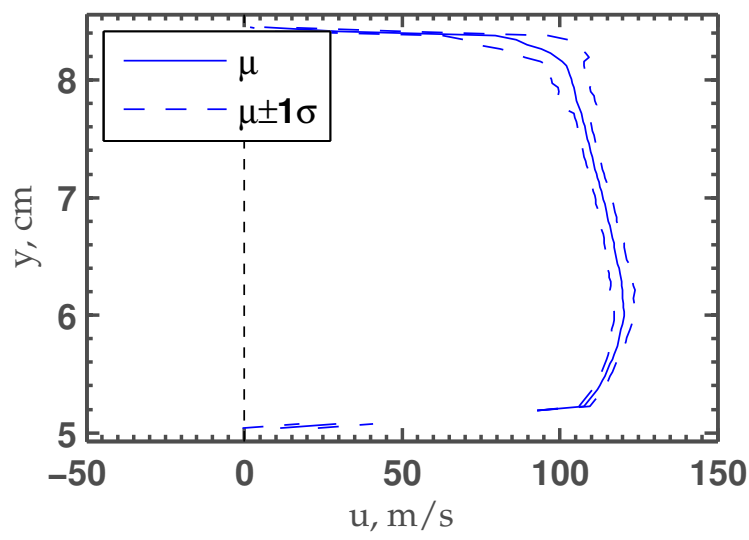

(b)

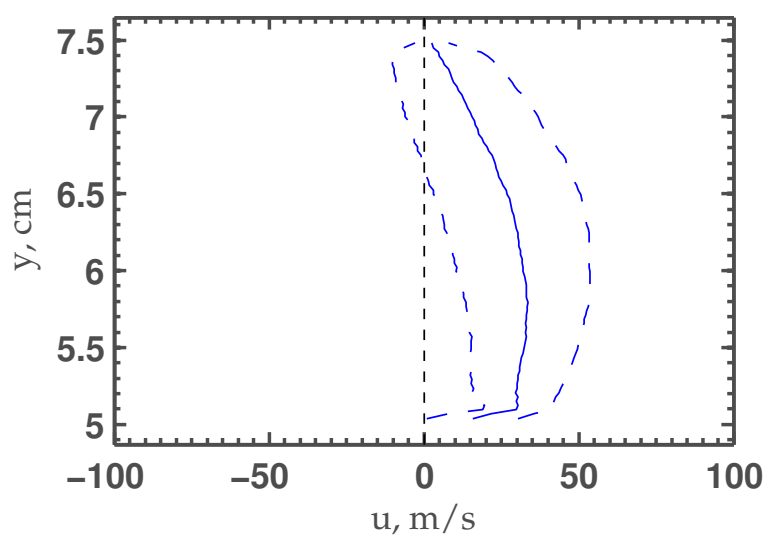

(d)

Figure 5.5: Mass flux control volume showing a) the control volume with superimposed normal velocity vectors and the normal velocity component and \pm 1 standard deviation for $b$ ) the incoming profile, c) the core exit profile, and d) the scavenge exit profile.

In contrast, the velocity profile of the scavenge flow (Fig. 5.5d indicates a mean velocity that varies considerably across the vertical plane, from nearly zero at the outermost portion to a value of about $30 \mathrm{~m} / \mathrm{s}$ closer to the splitter nose. This strong spatial variation in the mean flow is also linked with high velocity fluctuations as indicated by the large spread of the deviation bounds. The standard deviation along the entire profile is about $20 \mathrm{~m} / \mathrm{s}$ while the average flow velocity is only about $25 \mathrm{~m} / \mathrm{s}$. This indicates that a high degree of 
flow unsteadiness is occurring throughout the scavenge plan. The negative deviation bound (left dashed line) also shows significant negative velocities for $y>7 \mathrm{~cm}$. This indicates that recirculation is at these locations is present for a significant portion of time, but is highly intermittent.

\section{Control Volume Flux Analysis}

Based on the time-averaged surface-flow visualization by Barone et al. the centerline region of the flow was found to exhibit little or no span-wise variation. Thus, it is quite reasonable to apply time-averaged two-dimensional flow conservation principles to the centerline flow field. In order to conserve mass conservation through the system, the total flux through a control volume must be zero and this is found to reasonable for the mean velocity field. However, variations in the PIV data indicate that these principles are not reasonably applicable in the instantaneous sense. In particular, three-dimensional velocity components (e.g. due to turbulent fluctuations), can cause a non-zero instantaneous flux based on a control volume and velocity vector field which is two-dimensional. The quantification of this aspect is discussed below.

Figure 5.6 shows the results of the two-dimensional volume flux analysis for each instantaneous vector field. In Fig. 5.6a, it can be seen that the incoming and core fluxes are reasonably constant over time while the scavenge flux has a high variation. The latter result is consistent with the unsteadiness noted in Fig. 5.5d, indicating stable operation of the incoming of core flows and an unstable scavenge flow. The net flux shown in Fig. 5.6b and indicates that the variations of the scavenge flux are primarily responsible for the non-zero net flux values.

Figure 5.7 shows a histogram analysis of the data presented in Fig. 5.6. As indicated previously with the velocity profiles, the incoming air flux and core flux both have reasonably 


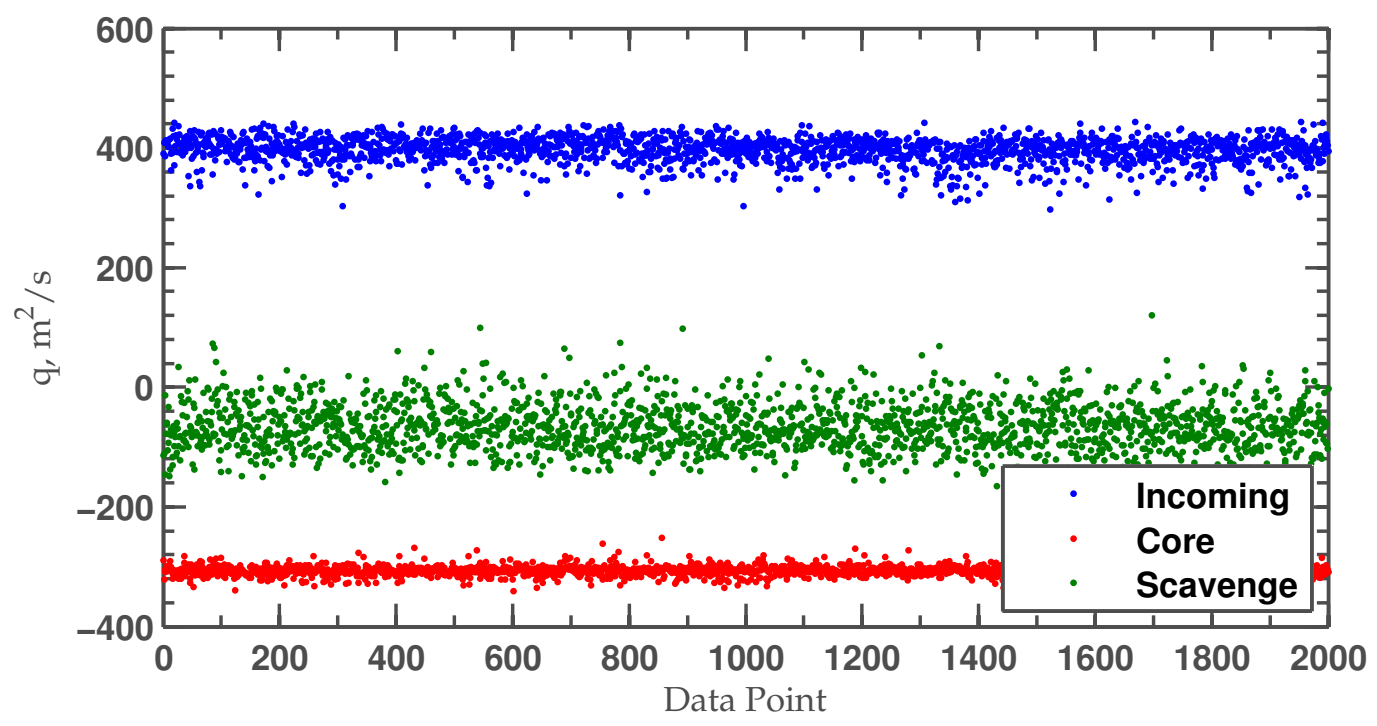

(a)

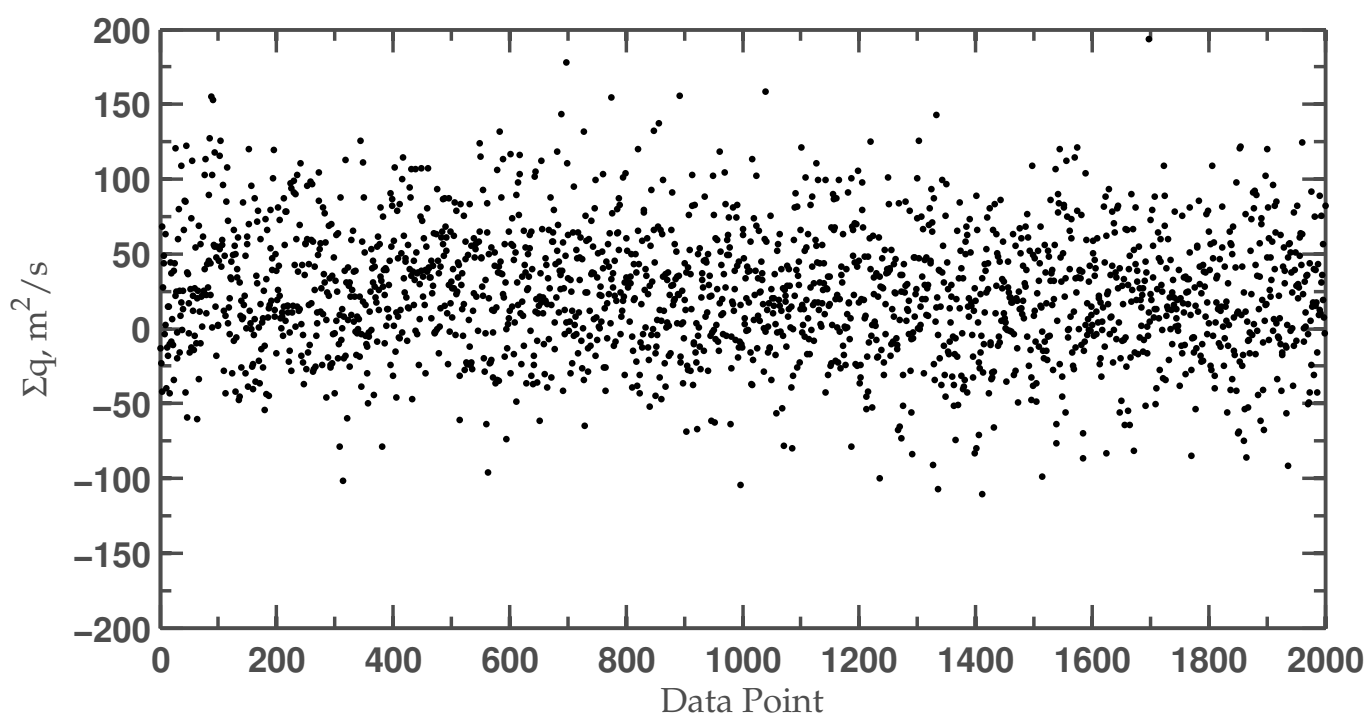

(b) Net flux

Figure 5.6: Local fluid volume flux scatter plot showing a) flux by component and b) the net flux. Note that flux is considered positive entering the control volume and negative exiting the volume. 
narrow distributions demonstrating stable operation. In contrast, the scavenge flux displays much larger deviations, including reversed flux (where flow is entering the control volume instead of leaving) for $5 \%$ of the time. Figure $5.7 \mathrm{~b}$ shows the net volume flux at each point, which is roughly symmetric about the zero-flux value indicating that the mean flow satisfies flow conservation and is approximately two-dimensional. Despite this two dimensionality in the mean net flux, there is significant scatter, nearly $25 \%$ of the total incoming flux. This substantial scatter is indicative of three-dimensional fluctuations. If one assumes the flow is nearly incompressible, then any non-zero net flux in the two-dimensional control volume must be counter-balanced by a spanwise flux. Comparing Figs. 5.7 and 5.7b suggests that these instantaneous span-wise fluxes are often on the order of the mean streamwise scavenge flux and thus very important to the overall flow dynamics.

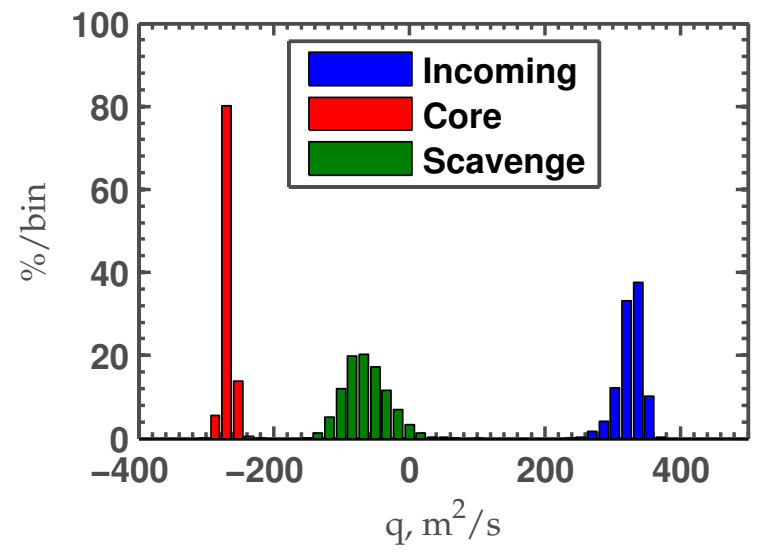

(a) Local flux by component

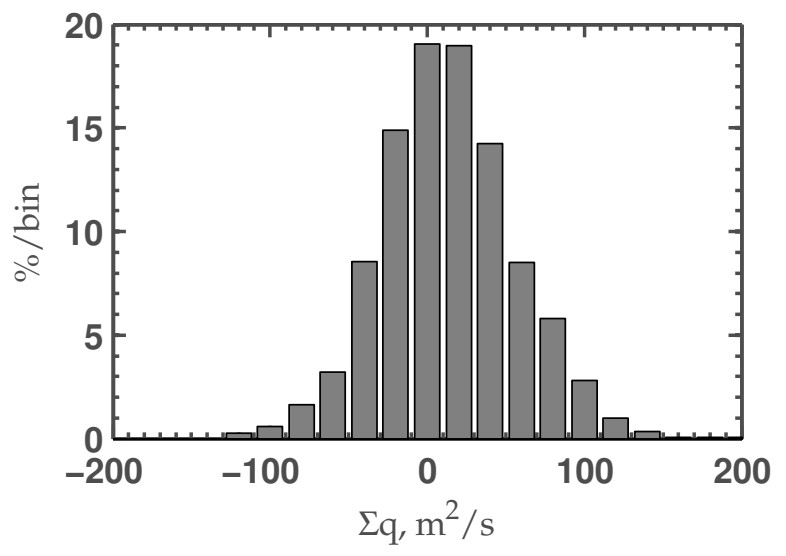

(b) Net flux

Figure 5.7: Control volume flux for OSG-1 (bin size of $15 \mathrm{~m}^{2} / \mathrm{s}$ )

To understand what is causing these large net flux variations, it is helpful to look at instantaneous PIV data fields. Several fields have been selected, representing different regimes that typically occur during IPS operation. Figure 5.8 includes three instantaneous flow fields 
that represent intermediate conditions in the IPS showing average, low, and high volume fluxes.

Figure 5.8a shows a case where the net volume flux is zero, satisfying the two-dimensional conservation of mass. This is representative of normal or mean operation, with predominately two-dimensional flow. There are small eddies and locations of slow flow, indicative of the recirculation zone seen in the previous oil-streak measurements. The second case in Fig. 5.8b shows that the scavenge flow leg is dominated by high-speed flow resulting in a high net flux out of the control volume (indicating that mass muss be entering through the spanwise plane). Strong events such as this have a significant impact when the instantaneous data are averaged, which could provide an explanation why the recirculation zone is not seen in the average flow field. In this case, neither the incoming flux nor the core flux is significantly altered during this event meaning that there must be non-uniform flow in the span-wise direction to satisfy conservation of mass.

Figure 5.8c shows a high net flux (indicating flow must be escaping in a three-dimensional plane). The flow field is characterized by reversed flow in the scavenge leg. Flow can be seen moving upstream from scavenge leg entering the core leg around the splitter nose effectively ingesting the scavenge flow path into the core flow path. As with the high net flux rate, there is little change in the incoming and core velocities fields. Recognizing that the histogram for the net flux has a Gaussian shape, it is a reasonable postulate that there are simultaneous spanwise occurrences of high net flux and low net flux. The net flux of these simultaneous events would cancel each other out satisfying conservation of mass in three dimensions. 

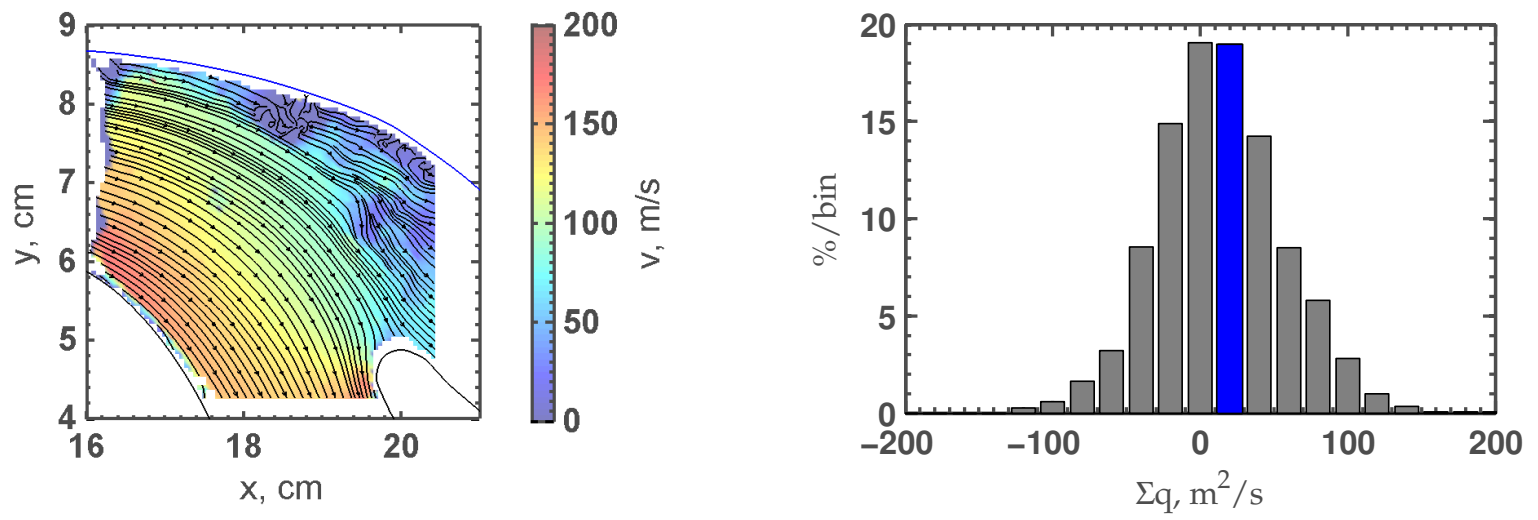

(a)
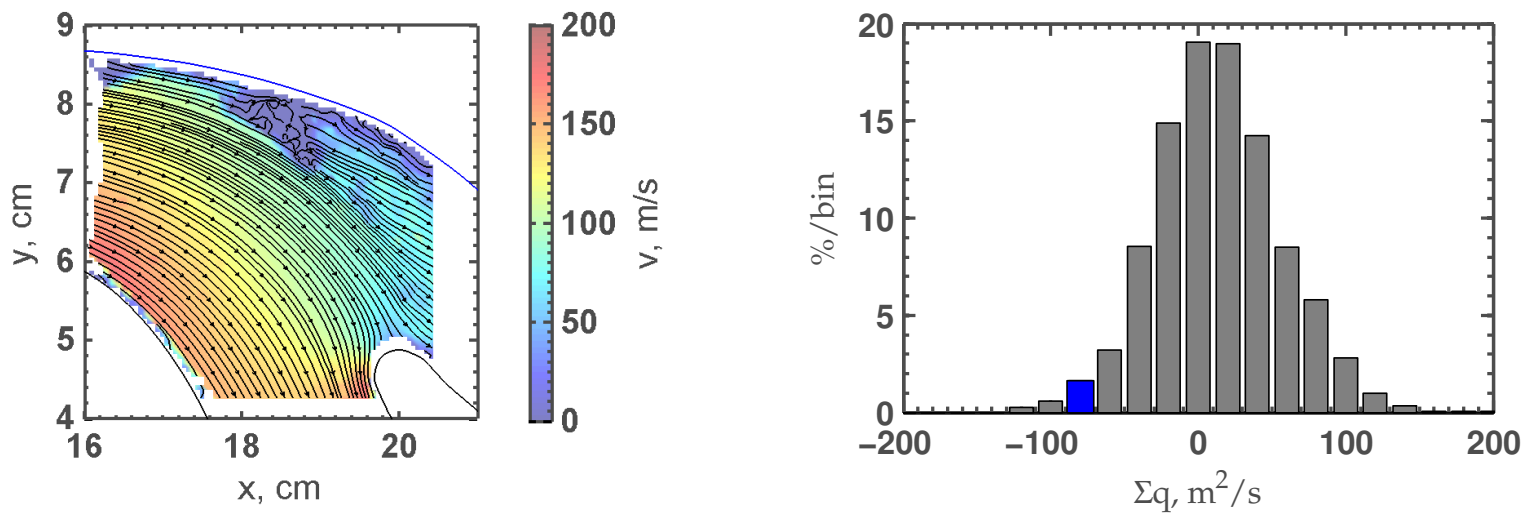

(b)
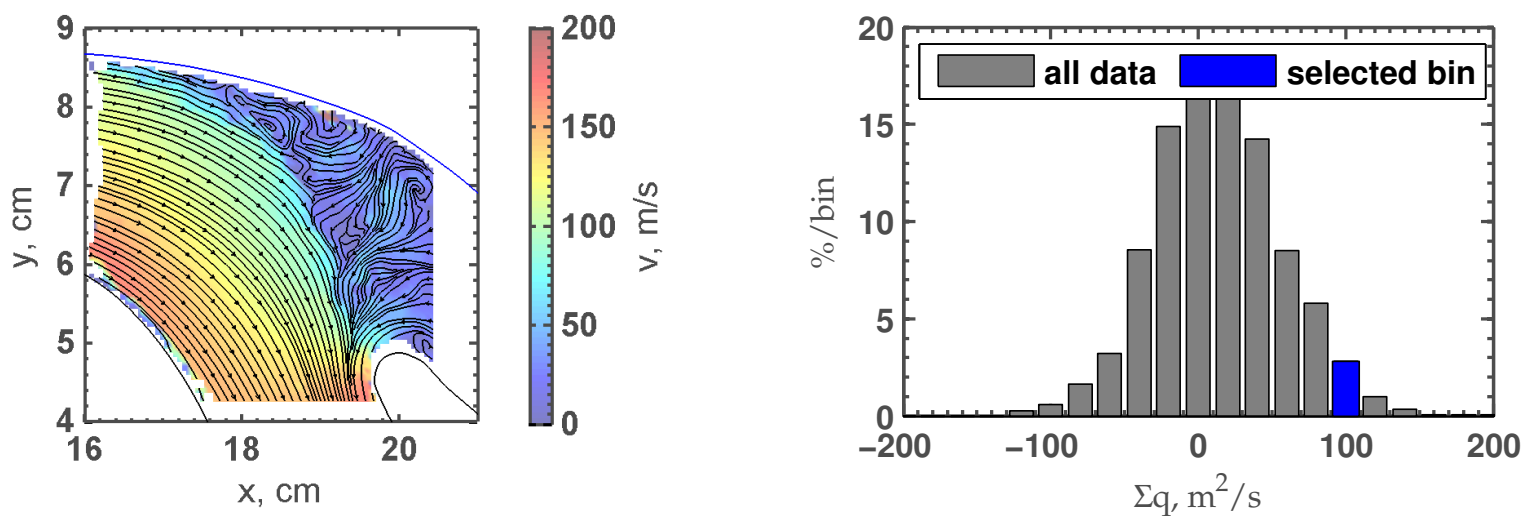

(c)

Figure 5.8: Instantaneous results for OSG-1 showing a) Mean control-volume flux, $\mu_{q}$, representative of mean IPS operation, b) Low control-volume flux, $\mu_{q}-2 \sigma_{q}$, caused by high-speed scavenge flow, and c) high control-volume flux, $\mu_{q}+2 \sigma_{q}$, indicative of ingested scavenge flow 


\subsubsection{OSG-2}

The PIV results for OSG-2 at $\beta=0.16$ are shown in Fig. 5.9. As opposed to OSG-1, the results for OSG-2 in Fig. 5.9a show the presence of a well-defined large recirculation zone in the average velocity field. This extensive recirculation zone is consistent with surface-streak visualization that indicated separation from $x=16.5 \mathrm{~cm}$ to $x=26.8 \mathrm{~cm}$. In Fig. $5.9 \mathrm{~b}$, there are a large deviations in the velocity at the entrance to the scavenge leg $(\approx 30 \mathrm{~m} / \mathrm{s})$, which is much higher than the deviations seen in OSG-1 $(\approx 15 \mathrm{~m} / \mathrm{s})$. The vorticity (Fig. 5.9c) in the flow shows that, although we now see the average recirculation, there is still significant shear present. Figure $5.9 \mathrm{~d}$ shows the useable vectors for the flow field, which is similar to OSG-1 showing a drop-offs upstream due to the laser sheet, along the walls due to reflections, and near the stagnation point along the OSG $(x=18.5 \mathrm{~cm})$ due to the accumulation of olive oil. For this case, wall reflections along the OSG were significant and the camera's field of view had to be adjusted so the reflections would not saturate the entire image. As a result, data is not available within $30 \mathrm{~mm}$ of the OSG but the main aspects of the flow field can still be seen.

The control volume flux calculations were performed for OSG-2. The velocity profiles in Fig. 5.10 for the incoming air and the core leg are nearly identical to those in Fig. 5.5 showing consistent steady operation in these regions. However, the scavenge flow mean velocity profile for OSG-2 has a clear reversed flow and higher flow velocity near the splitter than OSG-1. The deviation bounds of the OSG-2 scavenge profile are also much broader than for OSG-1, indicating increased unsteadiness for this geometry. The OSG operations are note entirely different, though. The data in Fig. 5.11 show similar results to OSG-1, with the incoming and core flows having very tight distributions and a much broader distribution for the scavenge flow. This is reflected in Fig. 5.11b where there is a similar Gaussian shape in the mass 


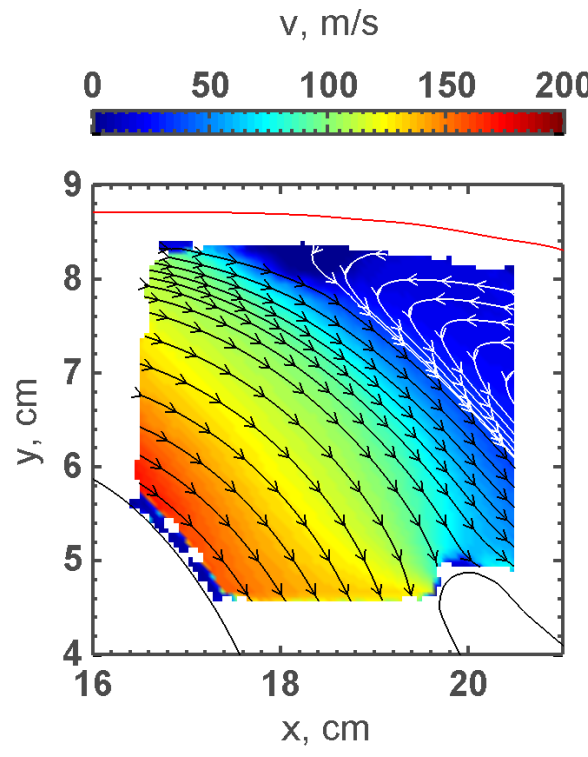

(a) Velocity Magnitude

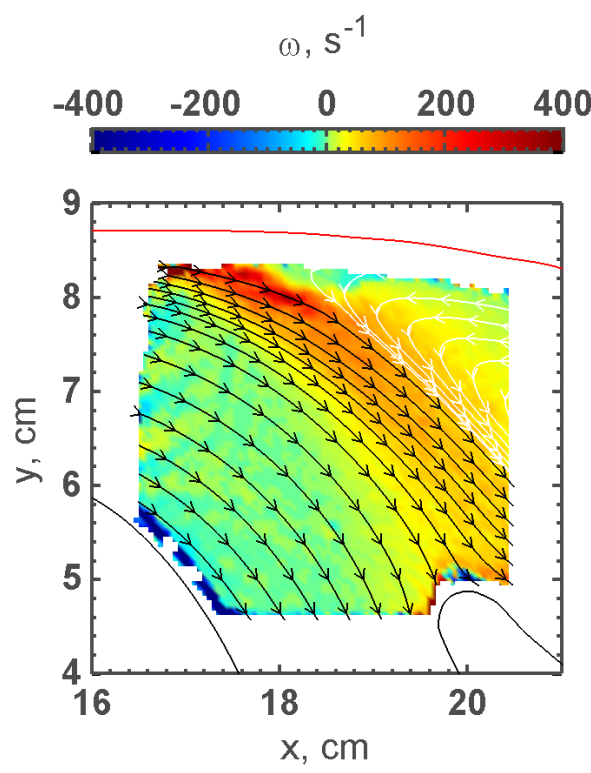

(c) Spanwise Vorticity

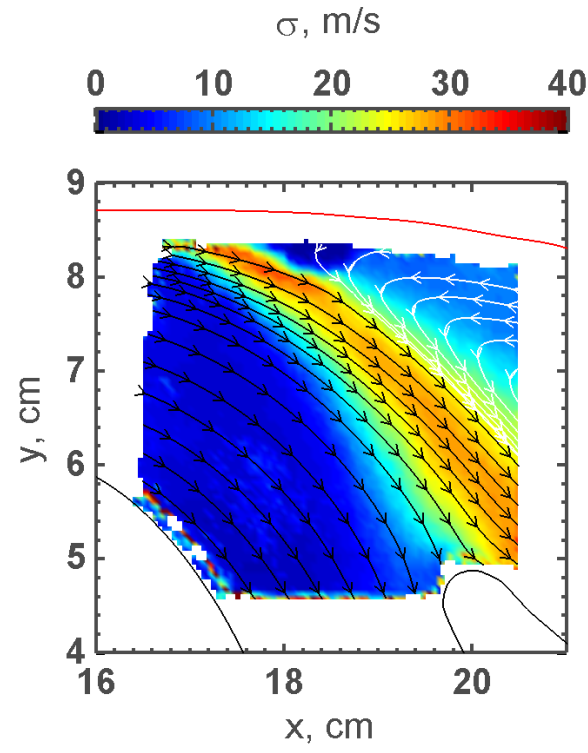

(b) Standard Deviation

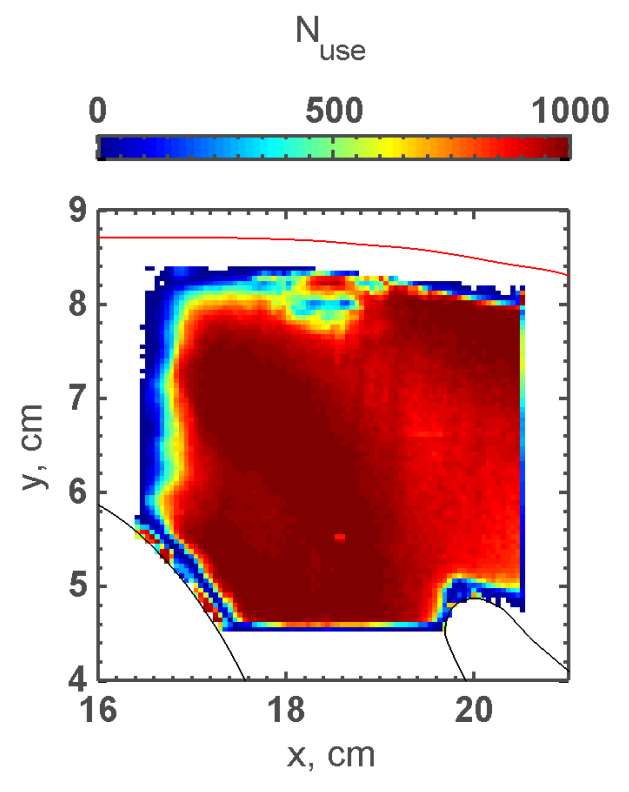

(d) Usable Vectors

Figure 5.9: Average quantities based on PIV measurements for OSG-2 at $\beta=0.16$ 
flux histogram, but the distribution of the net flux is much broader than the distribution of OSG-1.

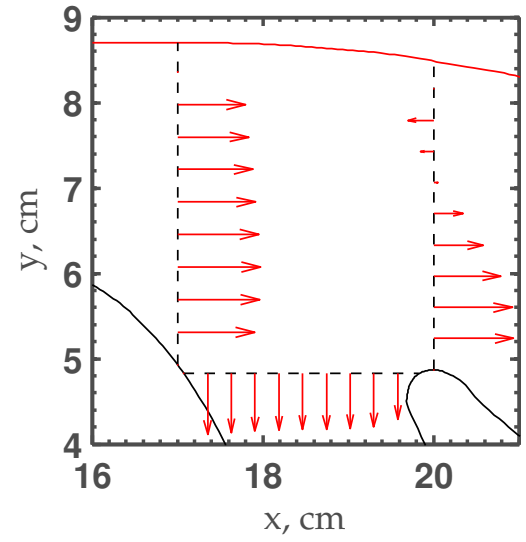

(a) Control Volume

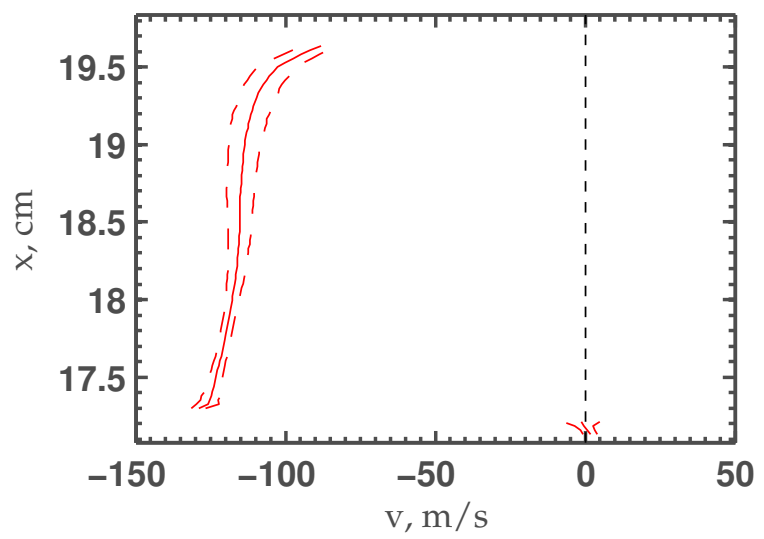

(c) Core Normal Velocity Profile

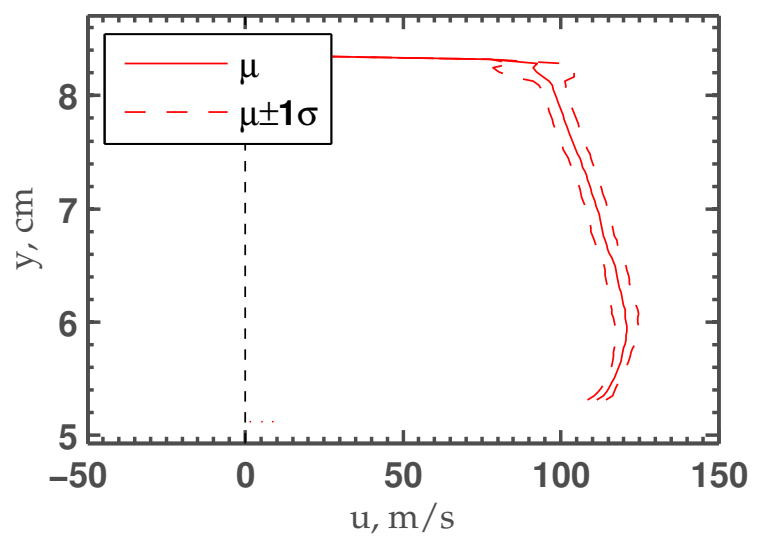

(b) Incoming Normal Velocity Profile

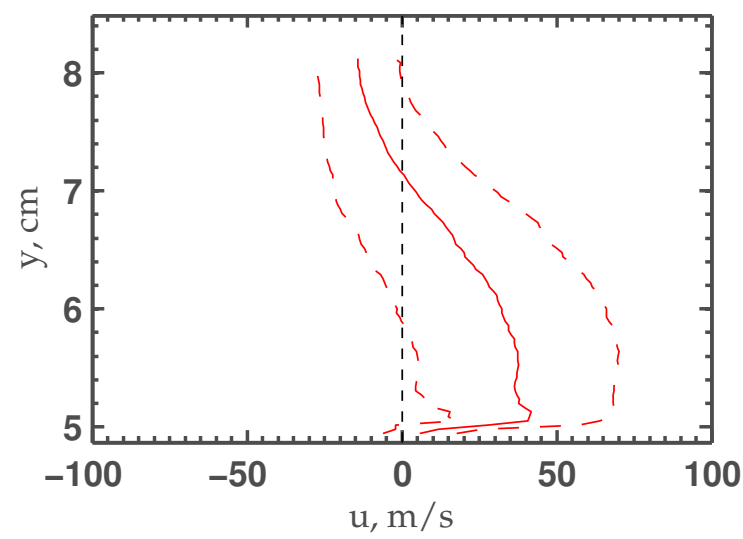

(d) Scavenge Normal Velocity Profile

Figure 5.10: Average normal velocity profiles for mass flux control volume

The instantaneous data for OSG-2 in Fig. 5.12 show that similar events that occur for OSG-2 that were observed in OSG-1. The mean scavenge volume flux (Fig. 5.12a) is representative of the mean flow. The low volume flux (Fig. 5.12b) is dominated by high-speed flow with minimal recirculation near the OSG. The high volume flux (Fig. 5.12c) is represented by flow being ingested into the core flow path. However, the broad distribution of the volume 


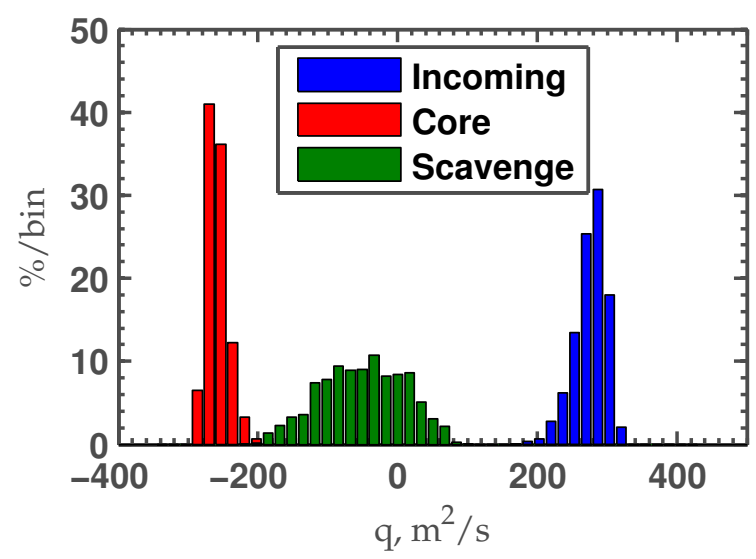

(a) Local flux by component

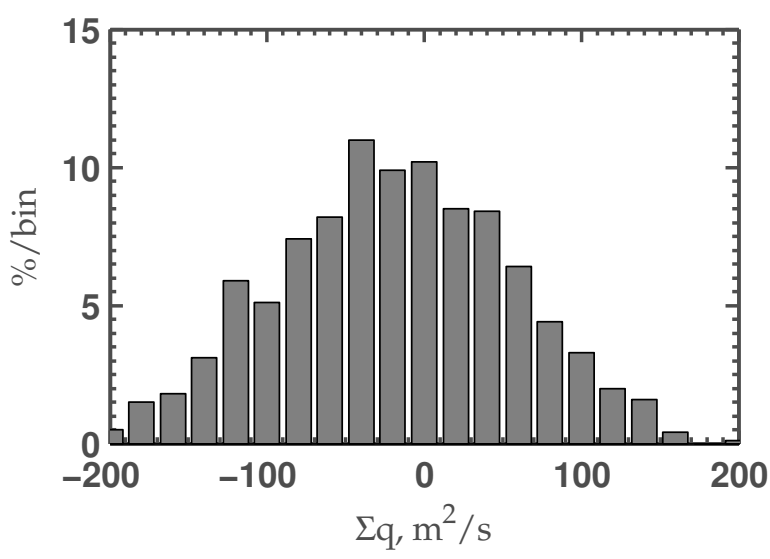

(b) Net flux

Figure 5.11: Control volume flux for OSG-2 (bin size of $15 \mathrm{~m}^{2} / \mathrm{s}$ )

flux suggests that these events occur with different frequencies, effecting lower separation performance. 

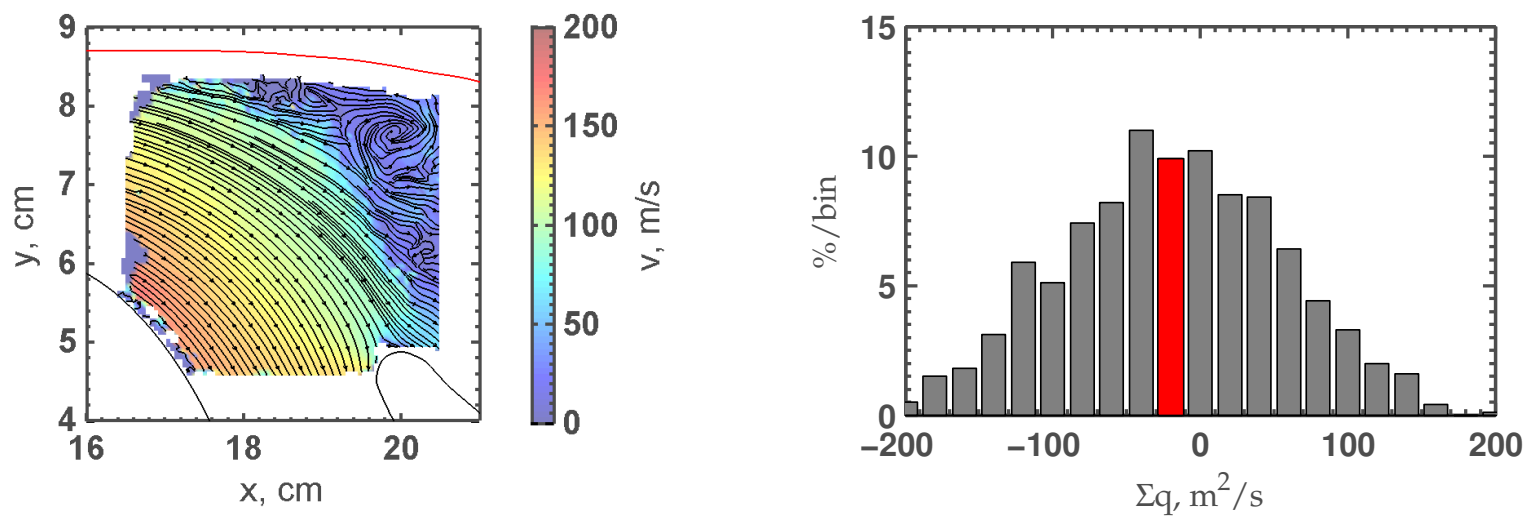

(a)
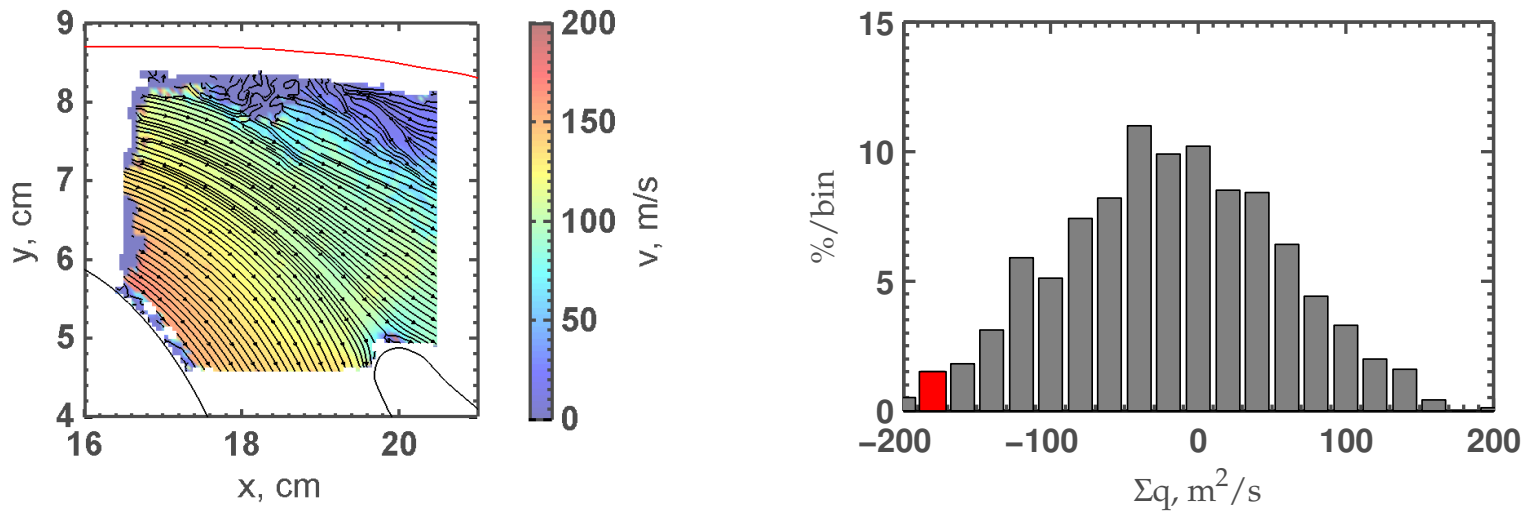

(b)
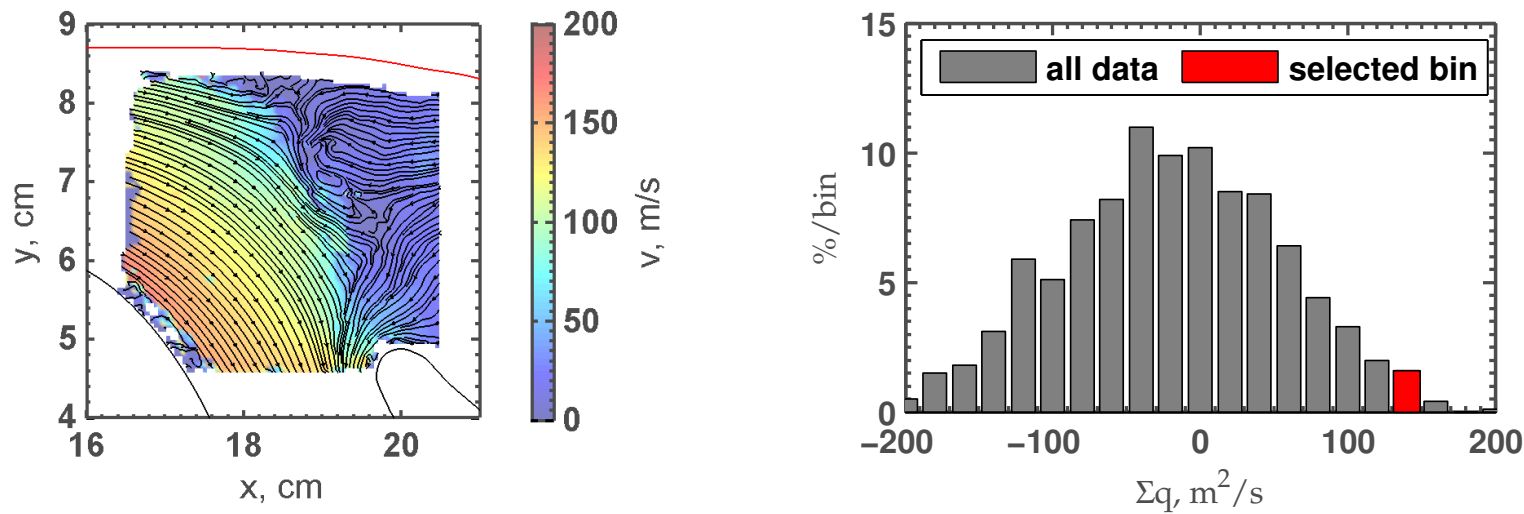

(c)

Figure 5.12: Instantaneous results for OSG-2 showing a) Mean control-volume flux, $\mu_{q}$, representative of mean IPS operation, b) Low control-volume flux, $\mu_{q}-2 \sigma_{q}$, caused by high-speed scavenge flow, and c) high control-volume flux, $\mu_{q}+2 \sigma_{q}$, indicative of ingested scavenge flow 


\subsubsection{OSG-3}

Results for OSG-3 are shown in Fig. 5.13. The recirculation zone is present in the velocity magnitude, but it is not as large as the recirculation zone found for OSG-2. This matches the oil streak results with the recirculation zone extending from $x=18.9 \mathrm{~cm}$ to $x=26.0 \mathrm{~cm}$. The standard deviation and vorticity figures also look similar to those found for OSG-1 and OSG-2, with large standard deviation and vorticity around the entrance to the scavenge flow leg and upstream of the recirculation zone. Figure 5.13d shows similar losses in vectors as those seen for OSG-1 and OSG-2, but OSG-3 shows a loss of usable vectors in the core flow region. This loss in vectors was caused by a seeding error, but has no impact on the calculations because there are more than enough vectors to resolve values for the mean and standard deviation in this region.

The control volume calculations are shown in Fig. 5.14. The field of view for OSG-3 is slightly downstream compared to the field of view for OSG-1 and OSG-2. This shift was employed to resolve more of the recirculation zone, but as a result there is a slight shift in the location of the incoming control volume plane. This does not affect the calculations significantly, as the incoming air and core leg profiles are similar to those seen for OSG-1 and OSG-2. The mean scavenge velocity profile is more similar to OSG-1 than to OSG-2 in shape, but more similar to OSG-2 in magnitude. The velocity profile shape of OSG-3 is similar to OSG-1, as there is only slight recirculation there is not a significant inflection as seen in OSG-2. However, there is larger velocity magnitude and broader deviation bounds near the splitter as with OSG-2. This information, in conjunction with the recirculation zone size and efficiency data from Barone et al. ${ }^{[5]}$, indicates that the size of the recirculation zone impacts the shape of the flux plane.

The properties of the net flux in Fig. 5.15 are consistent with those observed in OSG-1 


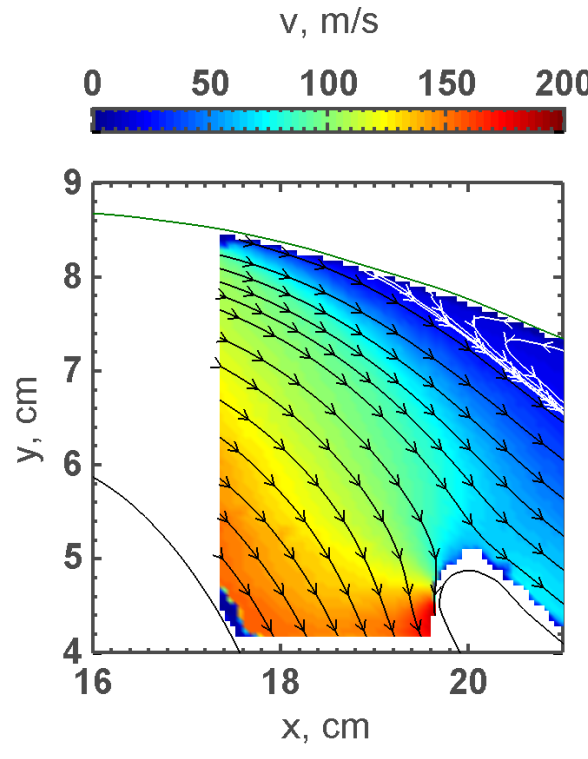

(a) Velocity Magnitude

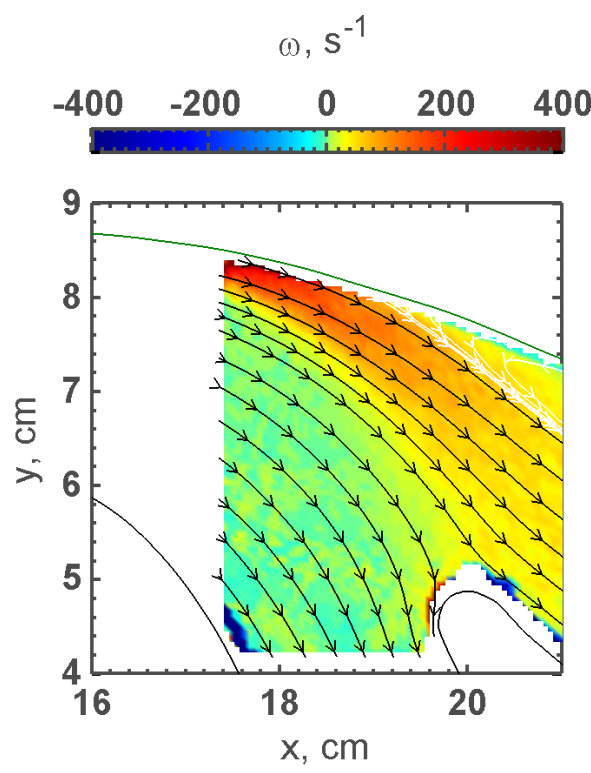

(c) Spanwise Vorticity

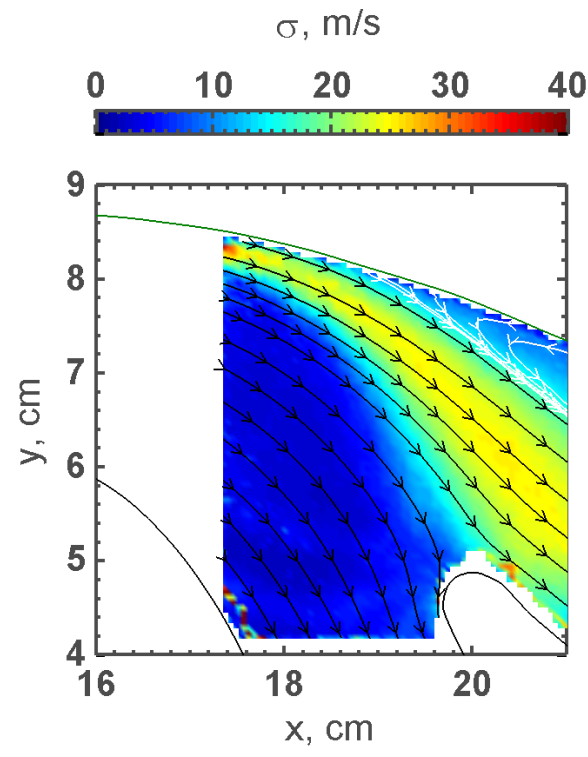

(b) Standard Deviation

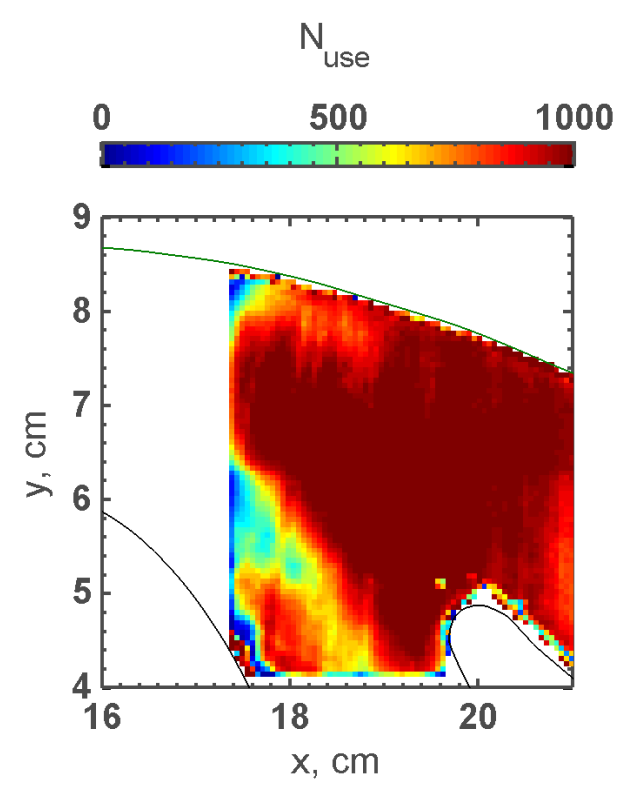

(d) Usable Vectors

Figure 5.13: Average quantities based on PIV measurements for OSG-3 at $\beta=0.16$ 


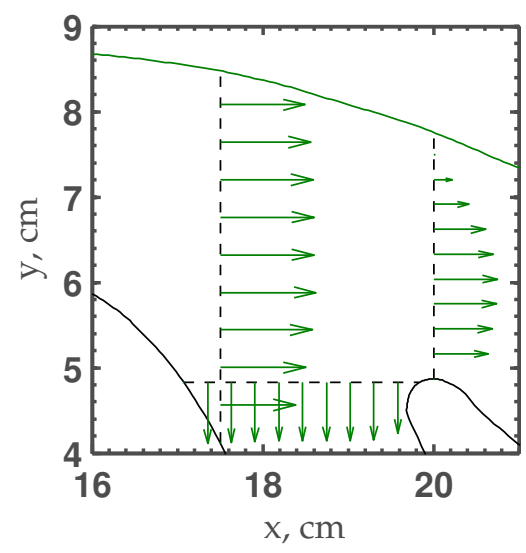

(a) Control Volume

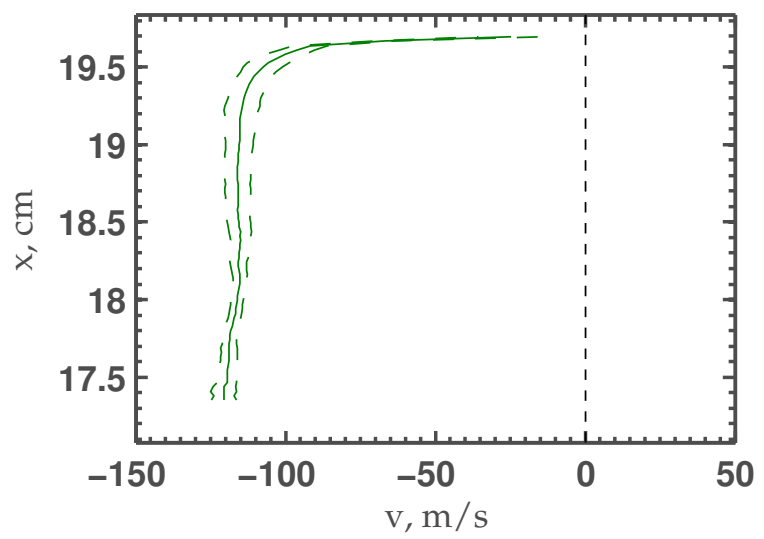

(c) Core Normal Velocity Profile

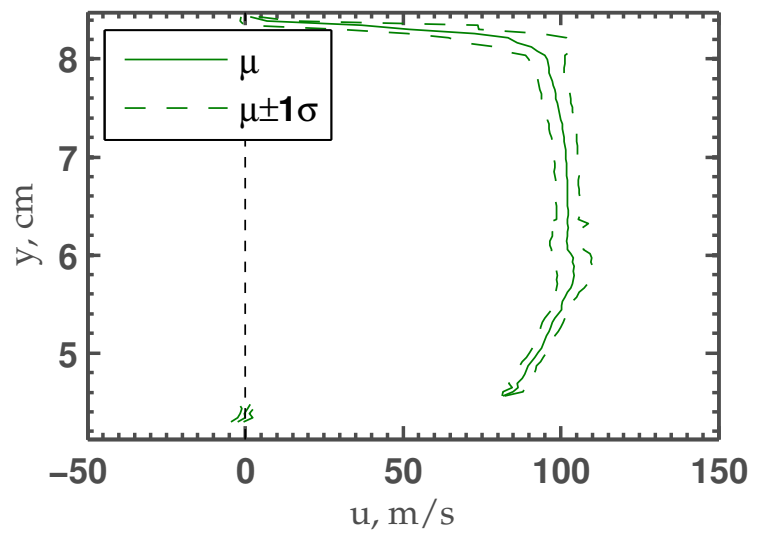

(b) Incoming Normal Velocity Profile

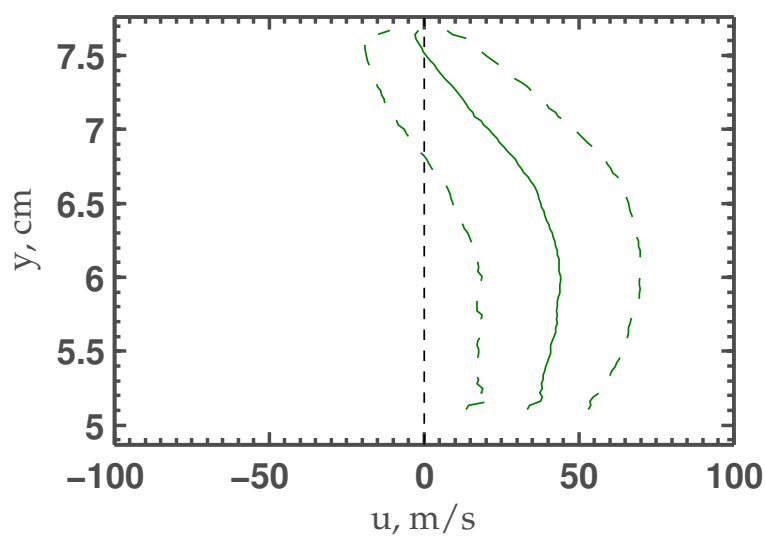

(d) Scavenge Normal Velocity Profile

Figure 5.14: Average normal velocity profiles for mass flux control volume

and OSG-2. In conjunction with scavenge profile comparison above, the net flux histogram (Fig. 5.15b) is closer in width to OSG-1 than to OSG-2, but is still broader than OSG-1. OSG-1 also has the highest efficiency, followed closely by OSG-3. This result indicates a correlation between high separation efficiency and low flow unsteadiness present in the bifurcation region.

The instantaneous results of Fig. 5.16 are also consistent with those observed in OSG-1 and OSG-2. Again, the mean scavenge volume flux (Fig. 5.16a) is representative of the mean flow. The low volume flux (Fig. 5.16b) is dominated by high-speed flow with minimal 


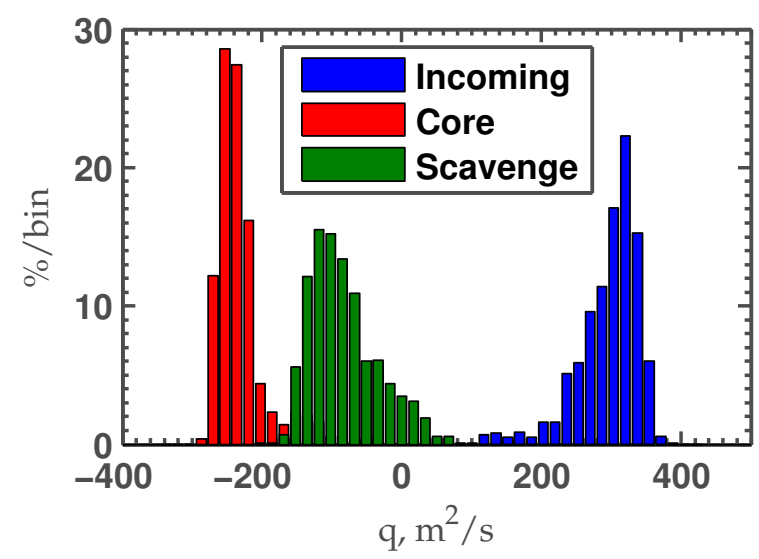

(a) Local flux by component

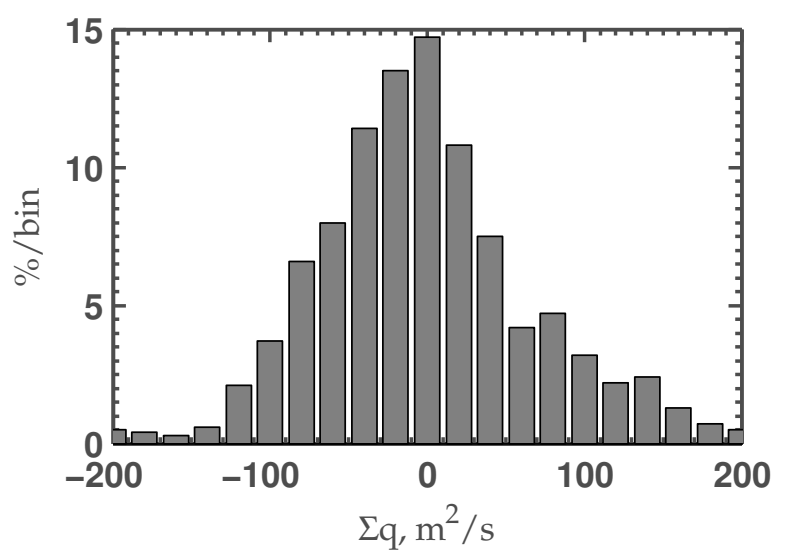

(b) Net flux

Figure 5.15: Control volume flux for OSG-3 (bin size of $15 \mathrm{~m}^{2} / \mathrm{s}$ )

recirculation near the OSG. The high volume flux (Fig. 5.16c) is represented by flow being ingested into the core flow path. 

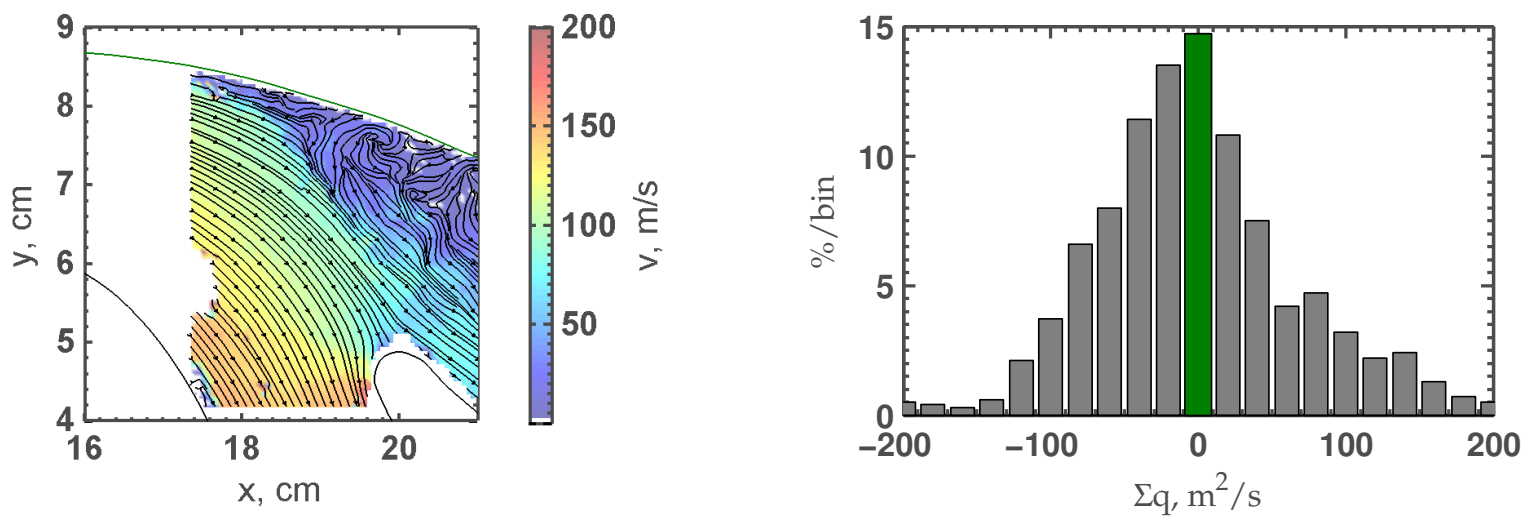

(a)
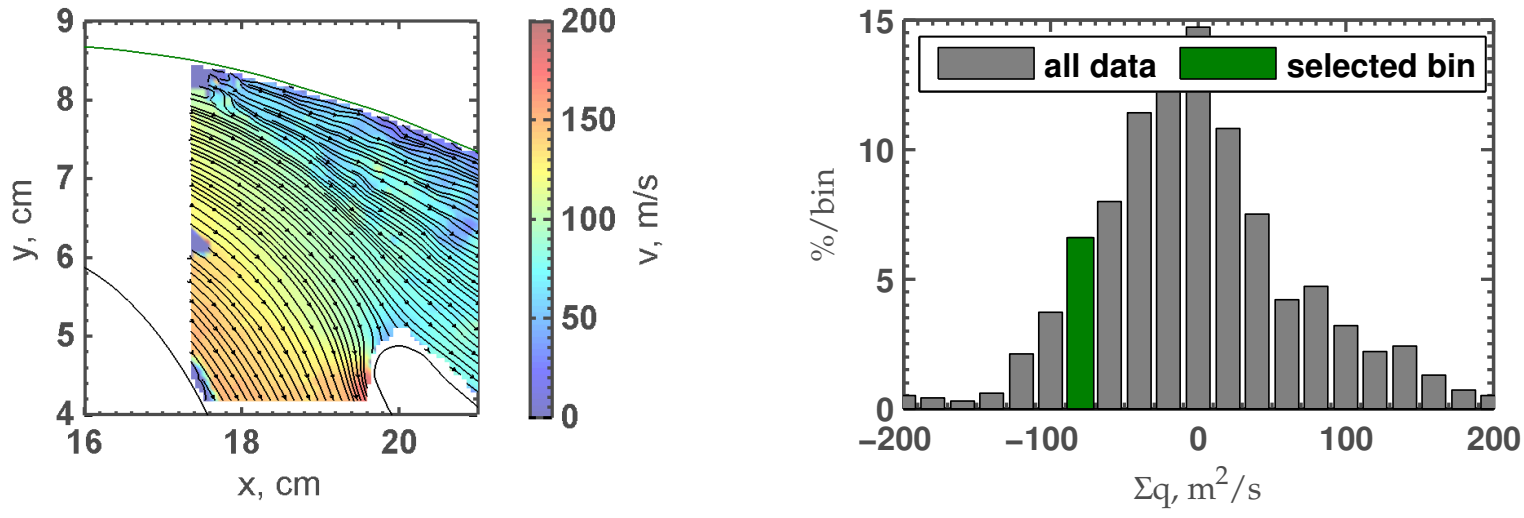

(b)
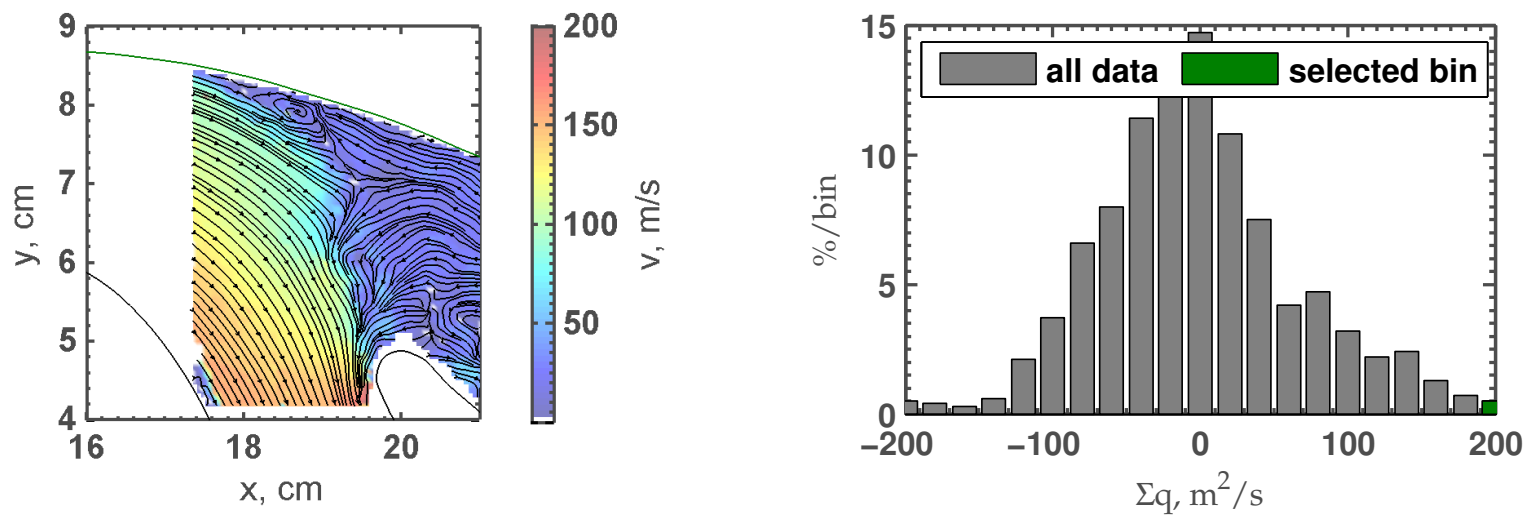

(c)

Figure 5.16: Instantaneous results for OSG-3 showing a) Mean control-volume flux, $\mu_{q}$, representative of mean IPS operation, b) Low control-volume flux, $\mu_{q}-2 \sigma_{q}$, caused by high-speed scavenge flow, and c) high control-volume flux, $\mu_{q}+2 \sigma_{q}$, indicative of ingested scavenge flow 


\subsubsection{High Speed Video}

To examine time dependent particle paths, high-speed video of the particles was acquired. Figure 5.17 shows two videos taken by the high-speed camera with selected frames are shown in Figs. 5.18 and 5.19. The movie in Fig. 5.17a and the still shots in Fig. 5.18 show an ingestion event where flow is pulled from the scavenge leg into the core leg, taking particles with it. An event like this typically lasts for only a few milliseconds but over the coarse of an IPS test, these events occur often, accounting for $25 \%$ of total operating time. Normal IPS operation is shown in Figs. 5.17a and 5.19 where most, but not all, of the particulate is entering the scavenge leg and any instabilities are confined to an area within the scavenge flow path. This type of flow pattern is the most prevalent, lasting 10 to 20 milliseconds and accounts for $50 \%$ of the operational time. The remaining $25 \%$ of operational time consists of events similar to the ingestion in Fig. 5.16a, but are not as severe. As shown by the steady stream of particles entering the core (even during normal operation), the inertia of the small particles is not enough to send all of them into the scavenge flow path. This type of inefficiency is not predicted in the steady-state model of Barone et al. ${ }^{[34]}$. This may explain, the inability of the model to account for low separation efficiency with these smaller inertia 10 micron particles. Therefore, unsteady effects, not considered in the model, are the cause of the decreased efficiency.

Figures $5.17 \mathrm{a}$ and 5.18 at frame 140 show that when flow is ingested into the core, a wall-normal vortex is formed around the y axis. More colloquially: it looks like a tornado is being drawn into the core flow. This vortex pulls particles from the scavenge flow leg into the core. The existence of this vortex is further evidence of the previous postulate that suggests that there is significant spanwise variation in the flow field. This "tornado" could be generated by spanwise gradients in the degree of flow reversal . In particular, the high 


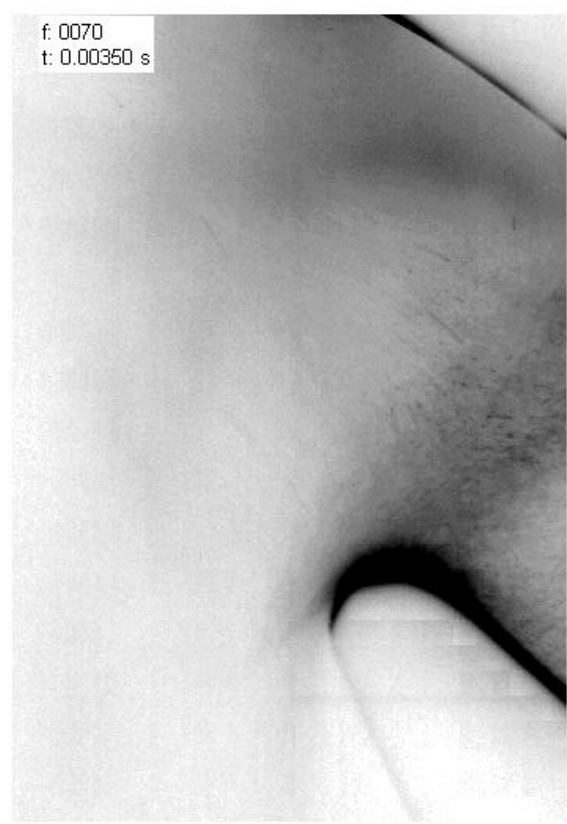

(a)

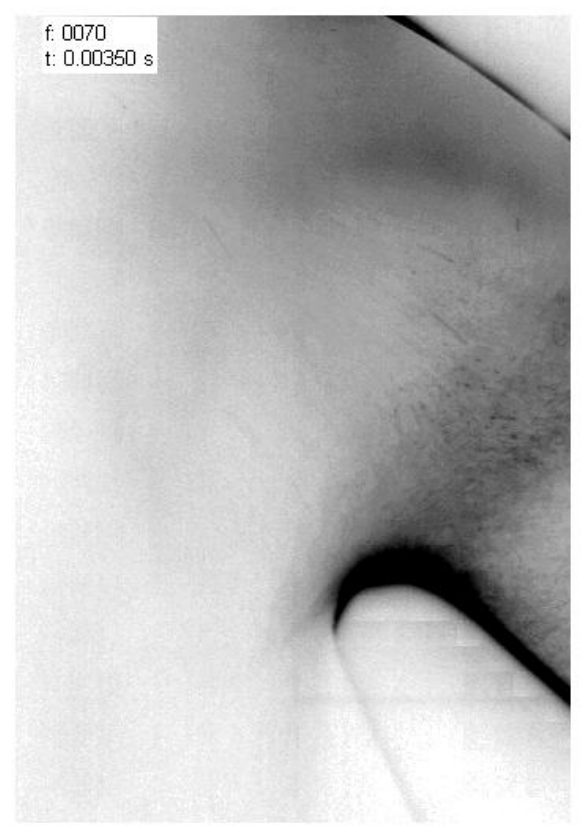

(b)

Figure 5.17: Embedded Video of OSG-1 with $10 \mu \mathrm{m}$ particles at $\beta=0.16$ showing a) and ingestion even and b) normal operation (click to play). Each video contains 180 frames at 20,000 frames per second, representing $9 \mathrm{~ms}$ of elapsed time. Note that Adobe Reader 9 or higher with Adobe Flash Player is required to play the video.

mean velocity from Fig. 5.8b next to the reversed flow in Fig. 5.8c would generate a wall normal vortex that could be detrimental to IPS operation. 


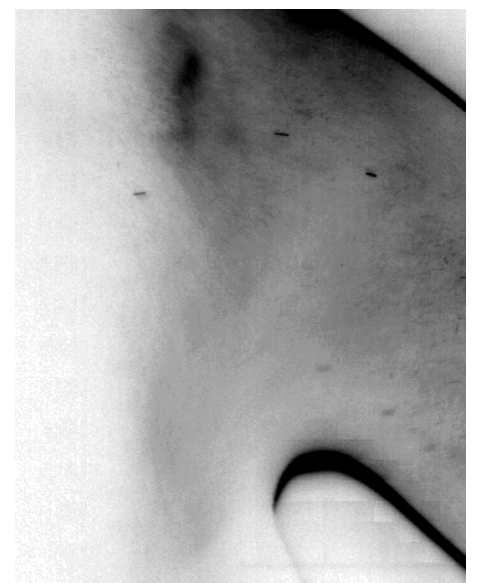

Frame: 140

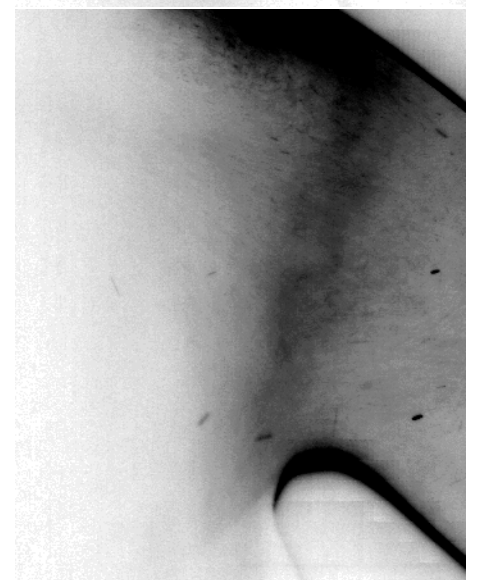

Frame: 149

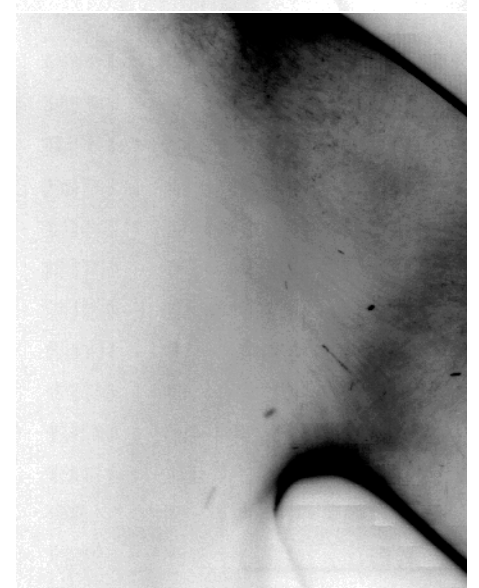

Frame: 158

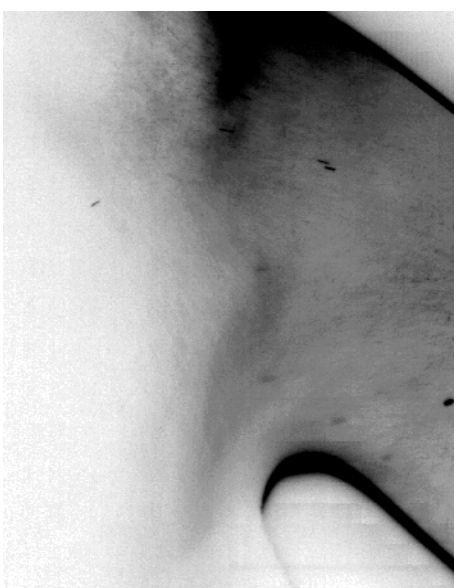

Frame: 143

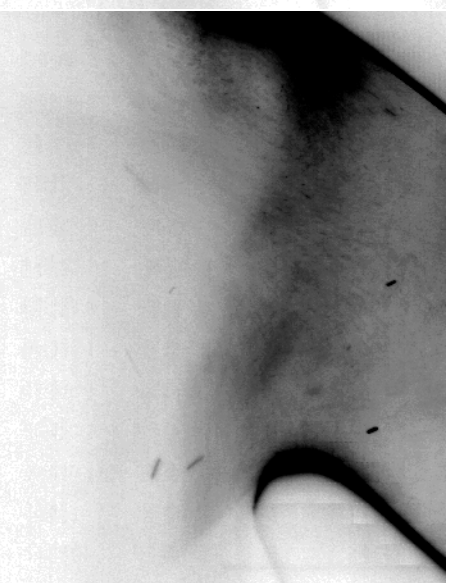

Frame: 152

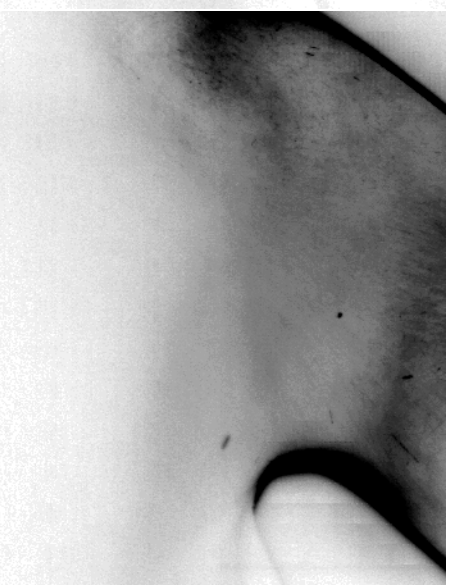

Frame: 161

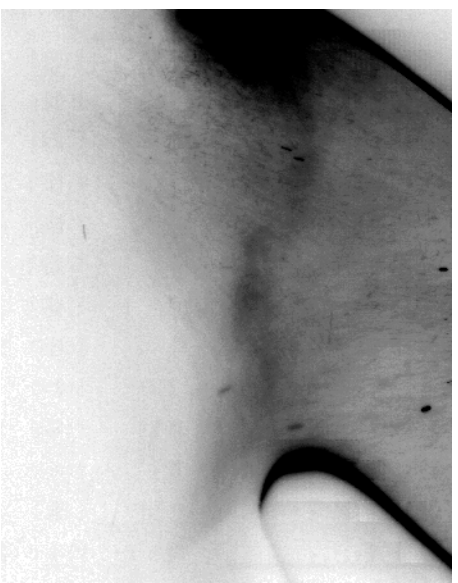

Frame: 146

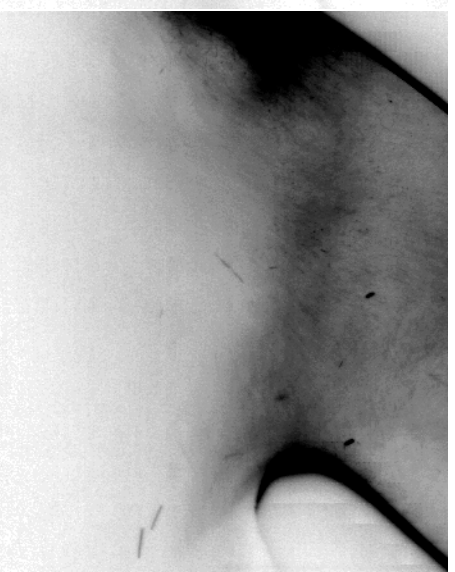

Frame: 155

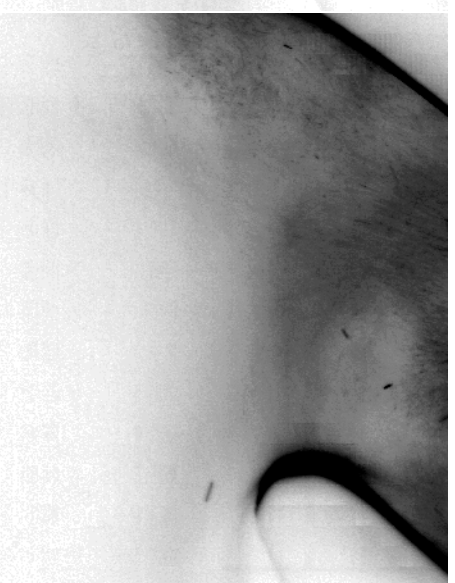

Frame: 164

Figure 5.18: High speed video results for OSG-1 showing ingestion event 


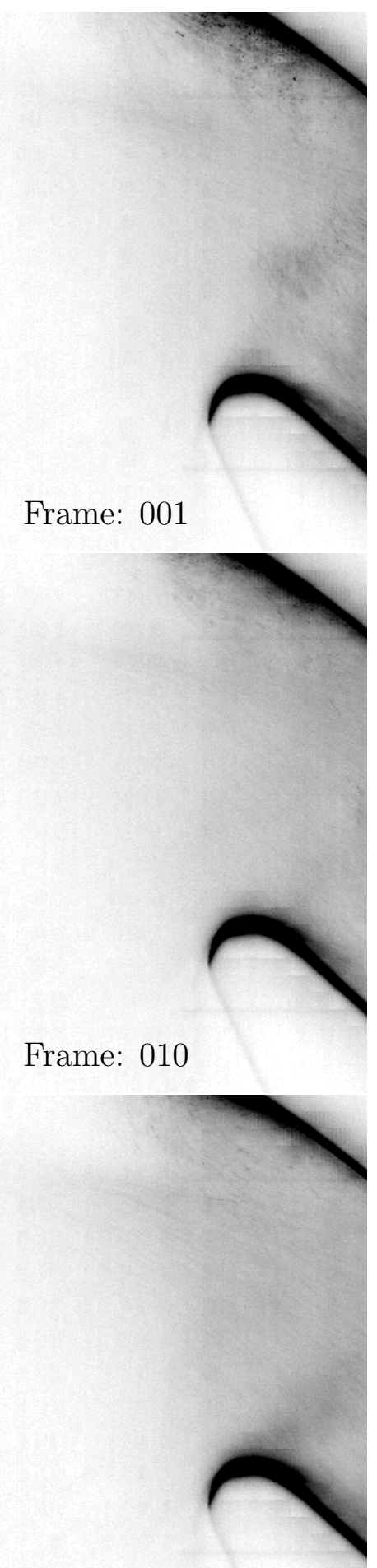

Frame: 019

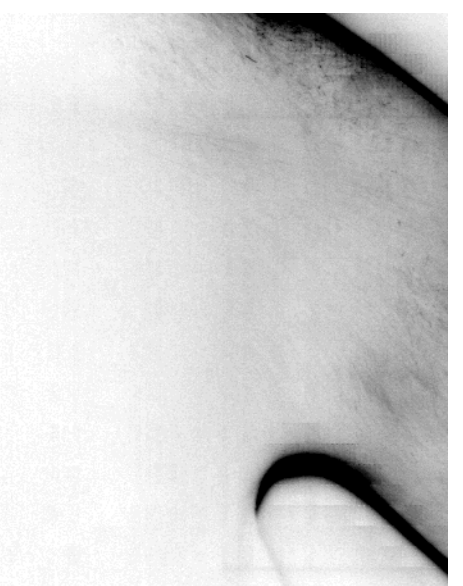

Frame: 004

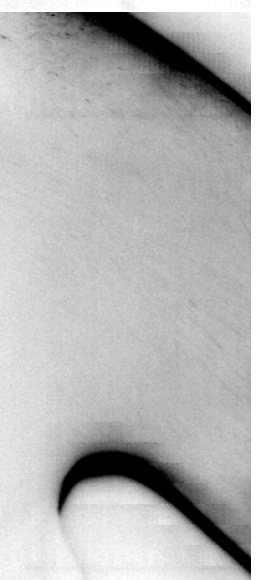

Frame: 013

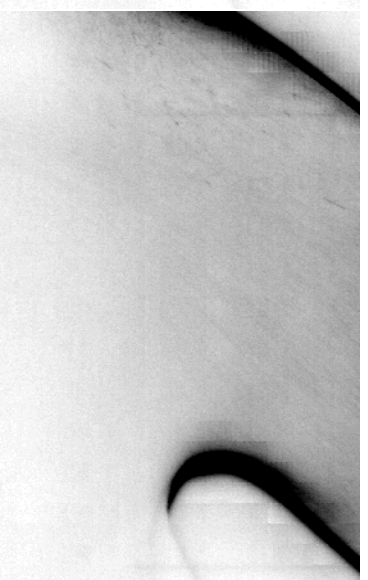

Frame: 022

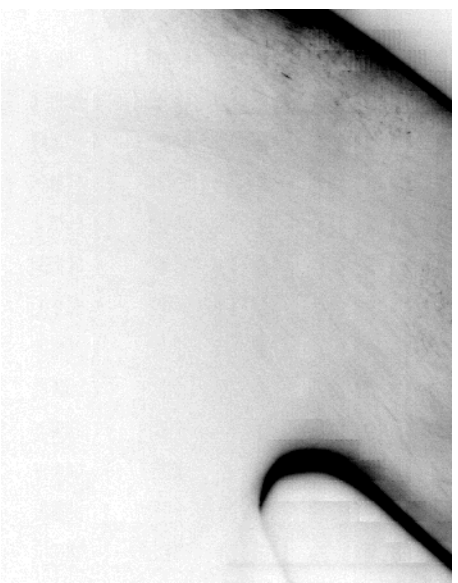

Frame: 007

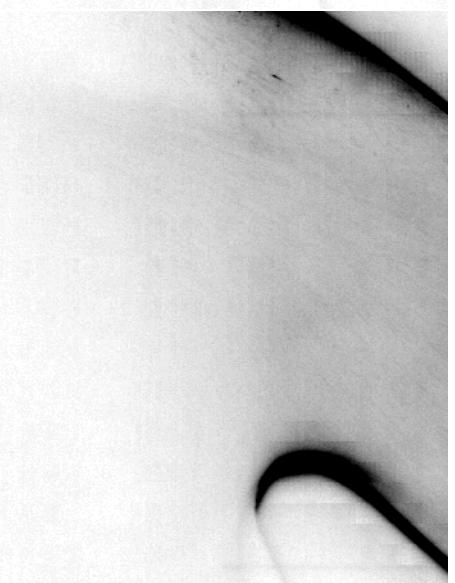

Frame: 016

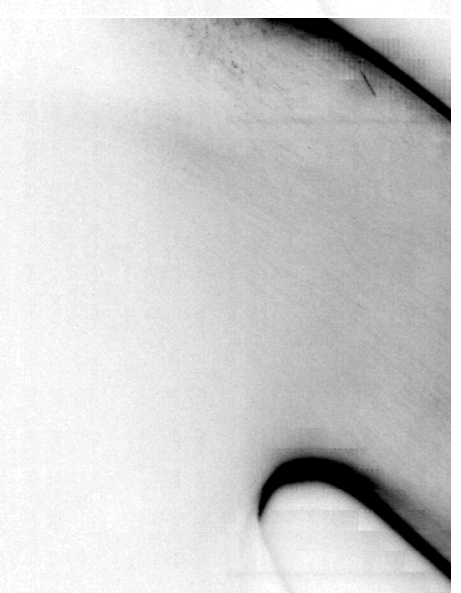

Frame: 025

Figure 5.19: High speed video results for OSG-1 showing expected operation 


\subsection{Turbulent Length Scale Discussion}

The turbulent length scale is a parameter used characterize the size of turbulent structures in a flow field. This length scale could be useful in the analysis of the fluid's effect on particles, as it can be combined with a time scale to obtain a Stokes number based on turbulence mentioned in Chap. 4. Typically, the turbulent length scale is obtained using a technique such as cross-correlation or proper orthogonal decomposition applied to large sets of PIV vector fields. While this study has the large data sets available, these techniques are difficult to apply even in the simplest flows. The flow present in the IPS here would be difficult to interpret as it contains many flow structures and bulk flow reversal, as seen in the data. Additionally, any length scales reported here would contain information in only two dimensions, whereas the high-speed video and PIV clearly indicate that there is three-dimensional turbulent motion in the regions of interest. However, a visual study of individual PIV snapshots show that the largest coherent turbulent structures tend to be on the order of $0.5 \mathrm{~cm}$ to $1 \mathrm{~cm}$, such as the one shown in Fig. $5.12 \mathrm{a}$ at $x=20 \mathrm{~cm}, y=7.8 \mathrm{~cm}$ which has an estimated radius of $0.6 \mathrm{~cm}$. This information could be useful in future analysis of the flow field, but true three-dimensional data is needed for a comprehensive study of turbulent length scales.

\subsection{Conclusions}

There are significant dynamic flow structures occurring within the IPS, shown by PIV and high-speed video measurements conducted in the research facility. The average flow fields for OSG-2 and OSG-3 match the previous oil-streak data for these geometries. OSG-1 does not indicate recirculation in the mean for the PIV field of view. However, there is recirculation in the instantaneous flow fields indicating incipient separation. Each OSG has a region of 
unsteady flux at the entrance to the scavenge flow leg in conjunction with a shear zone as indicated by the streamwise vorticity. This unsteadiness in the flow rate appears to cause the recirculation zone to be intermittent, especially for OSG-1.

Two-dimensional mass flux analysis of the bifurcation region showed instantaneous net gains and losses during IPS operation. This necessitates the existence of intermittent spanwise flux occurring during IPS operation as evident from the instantaneous results of the flow field, further showing that the scavenge flow leg is very dynamic. The instantaneous velocity fields indicate periods of what is considered "normal operation" (resembling the mean flow), periods where the scavenge leg is dominated by high velocity flow, and periods where the flow in the scavenge leg is reversed and ingested into the core flow leg. Each of the OSGs displayed the same intermittent behaviors, but each one had different characteristics in the scavenge flux plane. These differences correlate to the size and location of the recirculation zone, shown in oil-streak measurements, and to the measured separation efficiency of particles for each OSG.

High-speed video shows how the efficiency of small particles is being adversely affected by dynamic flow structures. It reveals that there is a wall-normal vortex forming meaning that there is span-wise variations in the flow field, hampering IPS efficiency for small particles. 


\section{CHAPTER 6}

\section{IPS Particle Dynamics}

Inertial Particle Separator (IPS) systems are installed in aircraft to minimize the dust and debris ingested by the engine. These ingested particles can be highly damaging to the internal components of a gas turbine engine, significantly reducing its lifetime ${ }^{[8-11,16]}$. Aircraft operating at low altitude in sandy areas are at increased risk, particularly helicopters, which lift particles off the ground, entraining them in the surrounding air due to rotor-downwash. In the extreme conditions of brownout, the particle concentrations can reach $2.5 \mathrm{~g} / \mathrm{m}^{3}$ obscuring vision and causing a high mass flux of particles to be injected into the engine inlets ${ }^{[27]}$.

IPS systems are generally designed to have high separation efficiency (fraction of particle removed from airstream entering an engine) combined with low-pressure losses ${ }^{[10]}$. To meet both objectives, the flow path (Fig. 4.1) must be carefully designed in terms of the amount and location of turning as well as the geometry of the flow bifurcation. The splitter geometry (which separates the core and scavenge flows) is key to controlling the flow and to direct the particle paths towards the scavenge flow. The Outer Surface Geometry (OSG) and the hub-geometry primarily dictate the degree of flow turning to achieve this scavenging. For 
many designs, the influence of the OSG can be especially important, as it can govern the recirculation zone that starts along its surface, just upstream of the splitter. Understanding how the flow reacts to these turns and the bifurcation of the flow path is critical to furthering IPS design.

In an effort to mitigate damage to gas turbine engines, there has been a significant amount of research done to understand the multiphase mechanics of particles within propulsion systems. Experimental work has primarily focused on the restitution and erosion properties of sand and dust within the engine, mainly relating to compressor and turbine blades ${ }^{[3,4,7,14-21]}$. Many computational studies have predicted the paths of particulate through an IPS system. These simulations typically employ potential flow or Reynolds-Averaged Navier-Stokes (RANS) solutions to the flow field. Particle paths are computed based on Lagrangian drag models and semi-empirical and probabilistic restitution models. The mass loading of particles in this type of flow have typically been considered to be small (even in the most severe brownout condition) so particle-particle interactions and the effects of the particles on the fluid are neglected.

Figure 6.1 shows an estimation of the particle paths based on several computational studies, the most recent is a study by Hamed et al. that used a RANS solution for the fluid flow field as well as a Lagrangian drag model and a probabilistic bounce model for particle paths. The $200 \mu \mathrm{m}$ paths (Fig. 6.1a) show that most of the particles (98.15\%) bounce through the IPS and into the scavenge flow leg. This generally agrees with the experimental data from Barone et al. ${ }^{[34]}$ that showed high efficiency $(>98 \%)$ of $120 \mu \mathrm{m}$ glass spheres for several IPS geometries. The paths for the $10 \mu \mathrm{m}$ sand particles (Fig. 6.1b) show the particles becoming entrained in the recirculation zone and are then pulled into the scavenge flow leg. The calculations by Hamed et al. ${ }^{[7]}$ estimate separation efficiency greater than $98 \%$ for particles larger than $7 \mu \mathrm{m}$, quickly dropping to $55 \%$ for $3 \mu \mathrm{m}$ particles and below $20 \%$ 
for $1 \mu m$ particles. The glass sphere experiments of Barone et al. ${ }^{[34]}$ show that particles with a $35 \mu \mathrm{m}$ diameter have an efficiency of $95 \%$ and $10 \mu \mathrm{m}$ particles have an efficiency of $85 \%$ in an IPS of similar design.

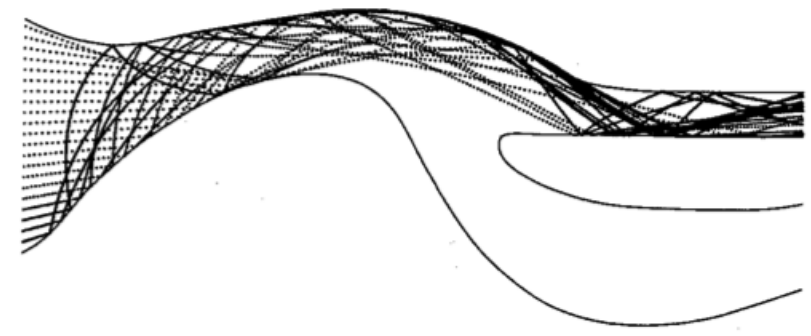

(a)

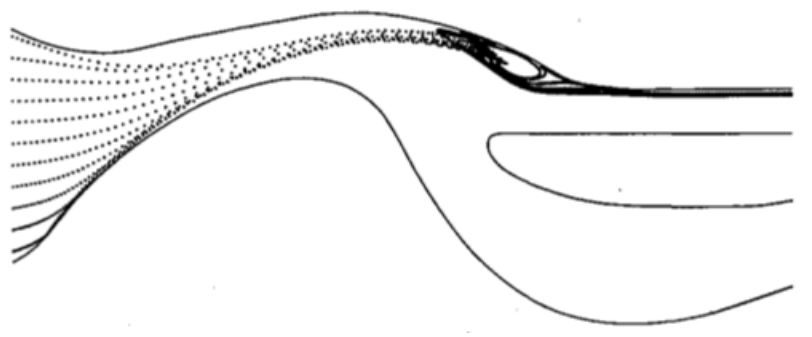

(b)

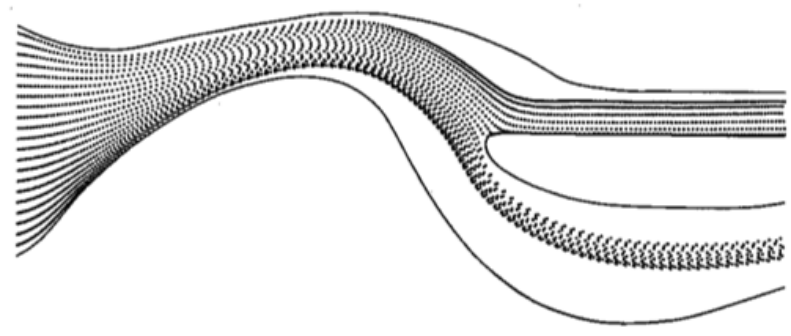

(c)

Figure 6.1: Calculations from Hamed et al. ${ }^{[7]}$ that calculated using RANS as well as semi-empirical drag model and probabilistic bounce models based on experimental data for a) $200 \mu \mathrm{m}$, b) $10 \mu \mathrm{m}$, and c) $3 \mu \mathrm{m}$ sand particles

Barone et al ${ }^{[32]}$ suggest that this loss separation efficiency is due to turbulent instabilities present within the scavenge flow leg and bifurcation region. Particle Image Velocimetry (PIV) was used to show that the flow field in the bifurcation region of the IPS experiences 
flow regimes that hinder particle separation. Using high-speed video, the influence of these dynamic events was shown to have significant effects on separation efficiency for $10 \mu m$ glass spheres.

Experimental data was deemed to be needed to quantify the mean and unsteady particle

dynamics in an IPS system ${ }^{[20]}$. The objective of this study is to elucidate the dynamics of $10 \mu \mathrm{m}$ and $35 \mu \mathrm{m}$ particles using PIV and provide an experimental comparison for future IPS computations. This has been carried out using a fundamental, two-dimensional flow facility designed for the full-scale particle and flow conditions.

\subsection{Methodology}

\subsubsection{Facility}

The experiments are conducted in a wind tunnel located at the University of Virginia designed to simulate the full-scale fluid velocities, length-scales, and particle sizes present in an IPS. To avoid the potential for gravitational bias toward the inner or outer surfaces, the wind tunnel is designed vertically so the airflow and particles are primarily directed downward. The tunnel also utilizes a two-dimensional geometry that represents the flow through a quarter annulus of a typical large helicopter engine while mitigating the influence of end-wall effects. The test section used in this study can be seen in Fig. 6.2 with the flow oriented in the downward direction. The two-dimensional geometry also eliminates the radial variations associated with inward-turning or outward-turning axisymmetric geometries, while representing a fundamental average flow path for both axisymmetric cases. The facility also allows different OSGs to be implemented. Currently, three geometries are used in the test section, denoted as OSG-1, OSG-2 and OSG-3 as shown in Fig. 4.3. The particle separation efficiency for these three 
geometries has been previously quantified in detail using $A 4^{[34]}$ test dust and several sizes of glass spheres ${ }^{[34]}$.

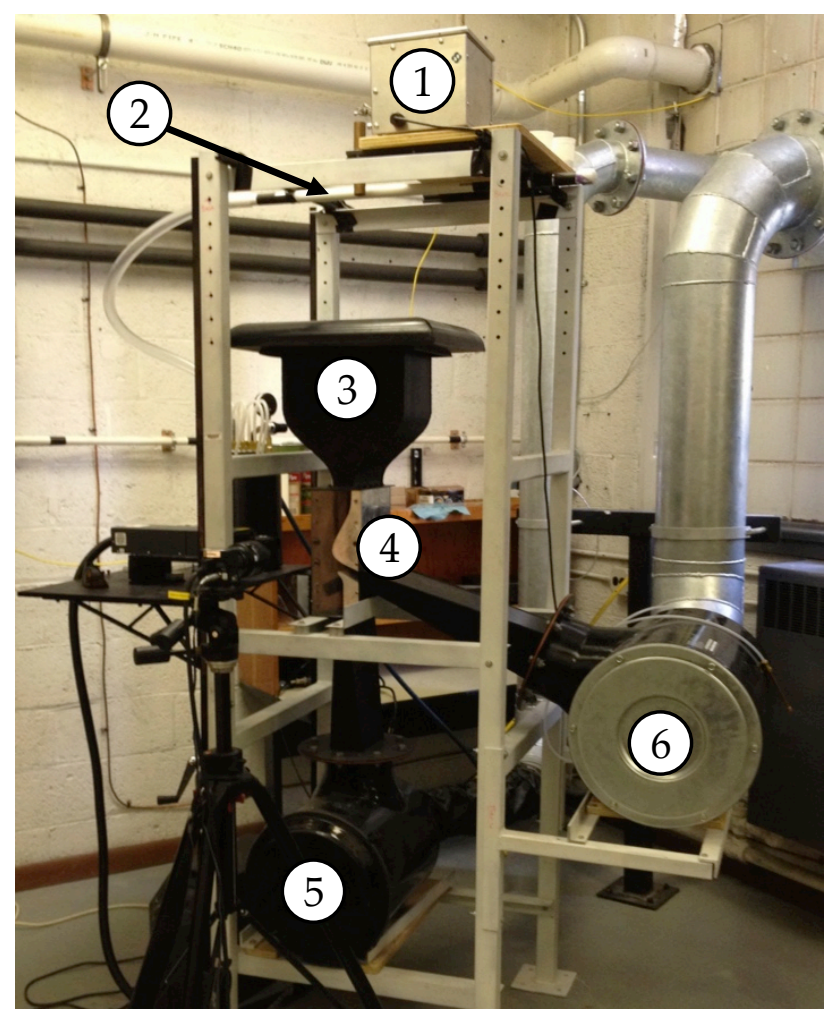

(a)

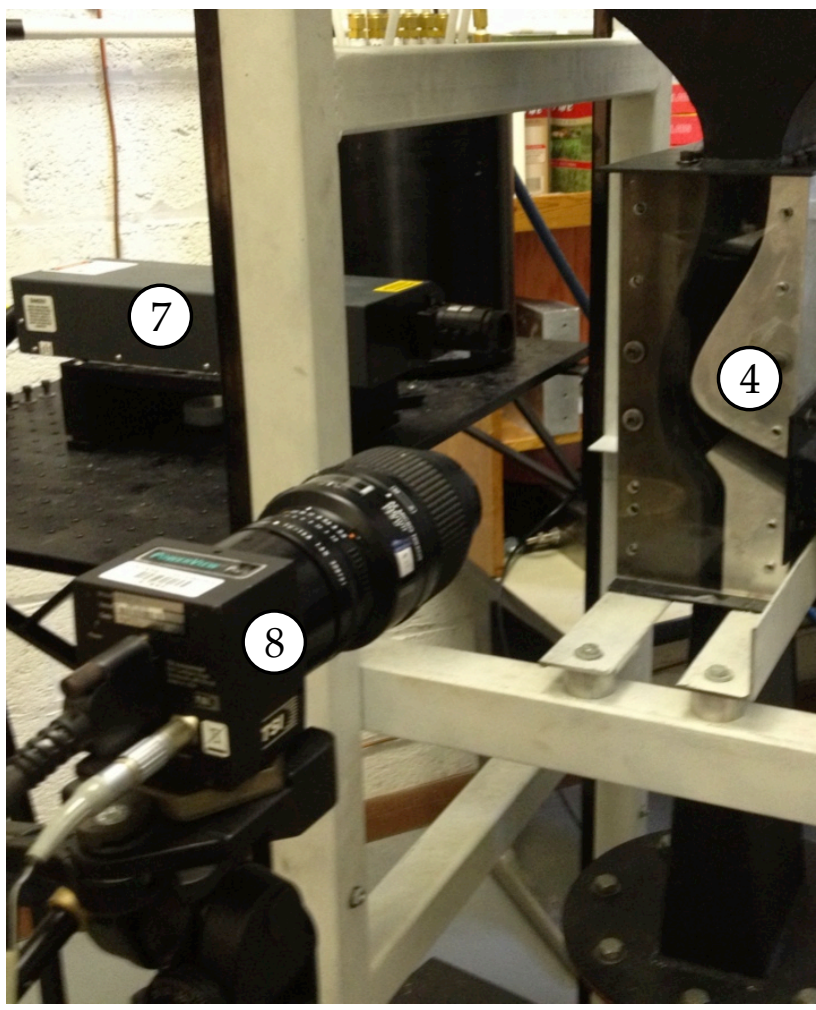

(b)

Figure 6.2: Experimental setup showing pictures of a) the wind tunnel showing the PDS (1), olive oil seed bar (2), tunnel air intake (3), test section (4), scavenge filter (5), core filter (6), and b) the PIV laser (7), and camera (8)

The IPS concept requires that air be removed from the flow through the scavenge leg of the system. The flow split, $\beta$, is defined as the ratio of the mass flow rate of air entering the scavenge leg relative to the total mass flow rate of air through the system. For this study the core mass flow was set at a constant $1.71 \mathrm{lbm} / \mathrm{s}$ and the scavenge flow was fixed at $0.326 \mathrm{lbm} / \mathrm{s}$ to yield the baseline flow split value of 0.16 . More details on the facility operation and optical setup are given by Barone et al. ${ }^{[8]}$ and Barone et al. ${ }^{[32]}$, respectively. 


\subsubsection{Multiphase PIV Measurements}

It is desirable to know the velocity of these particles traversing the flow and the same principles for flow tracking PIV can be used. Instead of using olive oil dtoples, particles are sized to match the sand particle sizes seen in an IPS rather than sizing particles to track the flow. This technique will be called MultiPhase PIV (MP-PIV) herein to distinguish the measurement from the fluid PIV measurements. The MP-PIV measurements reported here are focused on the bifurcation region of the IPS and within the center of the test section in the spanwise direction. This region, between $x=17 \mathrm{~cm}$ and $x=20 \mathrm{~cm}$, is where the flow is diverted into the core and scavenge flow legs.

Two sizes of glass-spheres with nominal diameters of $10 \mu \mathrm{m}$ and $35 \mu \mathrm{m}$ were measured using MP-PIV. These particle sizes were selected because they are representative of A4 test dust; the $35 \mu \mathrm{m}$ particles represent the mean and the $10 \mu \mathrm{m}$ represent the smaller sizes that have shown a decreased separation efficiency ${ }^{[34]}$. Glass spheres were chosen to eliminate the effects associated with non-spherical particles, such as variations in the drag coefficient and coefficient of restitution. This simplification will aid in modeling and analysis of the data. Particles were added to the flow using the Particle Delivery System (PDS) detailed in previous work ${ }^{[8]}$. This system uses an auger feeder combined with an air ejector to deliver a cloud of particles into the inlet of the tunnel at $0.050 \mathrm{~kg} / \mathrm{min}$.

Table 6.1 gives details of the particles used in the MP-PIV measurements, including separation efficiency at $\beta=0.16$, mean particle diameter $\left(d_{\text {mean }}\right)$, the diameter for which $97.5 \%\left(d_{97.5 \%}\right)$ of particles are smaller, and the diameter for which $2.5 \%\left(d_{2.5 \%}\right)$ of particles are smaller ${ }^{[34]}$. Due to the inherent nature of an IPS to separate particles from the core stream, the glass spheres are not uniformly distributed through the flow field as would be achieved using sub-micron tracer particles. The non-uniformity produces a region in the 
Table 6.1: Description of particles used in MP-PIV measurements

\begin{tabular}{ccccc}
\hline Nominal Size & $\eta_{\beta=0.16}$ & $d_{\text {mean }}$ & $d_{2.5 \%}$ & $d_{97.5 \%}$ \\
\hline $10 \mu m$ & 0.85 & $8.4 \mu m$ & $2.9 \mu m$ & $16.6 \mu m$ \\
$35 \mu m$ & 0.95 & $24.8 \mu m$ & $5.7 \mu m$ & $61.9 \mu m$ \\
\hline
\end{tabular}

flow field where very few vectors can be resolved because there are no particles present. For PIV measurements, 30-50 valid vectors are required to accurately predict the mean velocity; however, it is useful to know the position and velocity of particles in the flow field, even if the data are not converged. As a result, data are reported when at least $1 \%$ of the image pairs have vectors, e.g. for a set of 2000 image pairs, at least 20 valid vectors are required to be considered as a valid data point. The associated useable vector count is included for all measurements to indicate the significance of each point.

\subsubsection{Particle Restitution}

As particles increase in size, their inertia will carry them into the walls at significant velocities. When a particle impacts a wall, there is a transfer of energy between the particle and wall. When the particle leaves the wall (bouncing off) much of its momentum will have reversed direction, but some will be lost to viscoelastic effects. Figure 6.3 shows a diagram of a particle impact. The particle has an incoming velocity $\vec{v}_{i n}$, with a normal component $\vec{v}_{n, i n}$, a tangential component $\vec{v}_{t, i n}$, and angle $\theta_{i n}$. After the particle collides with the wall, a similar nomenclature is used and values are designated by the out subscript. This particle leaving the wall will have less momentum than it did before impact, characterized by the ratio of incoming to outgoing momentum called the Coefficient of Restitution $\left(C O R_{\hat{n}}\right)$. For this study only the momentum loss in the wall normal direction will be considered, shown in the Eqn. (6.1). 


$$
C O R_{\hat{n}}=\frac{\left|\vec{v}_{n, \text { out }}\right|}{\left|\vec{v}_{n, \text { in }}\right|}
$$

Knowing how these larger particles are bouncing off of the walls is vital in understanding the mechanisms of efficiency in an IPS. Of course, there are many other factors other than restitution (e.g. particle shape), but the use of spherical particles provides the unique opportunity to measure the normal coefficient of restitution independent of shape effects influencing the manner a particle will bounce.

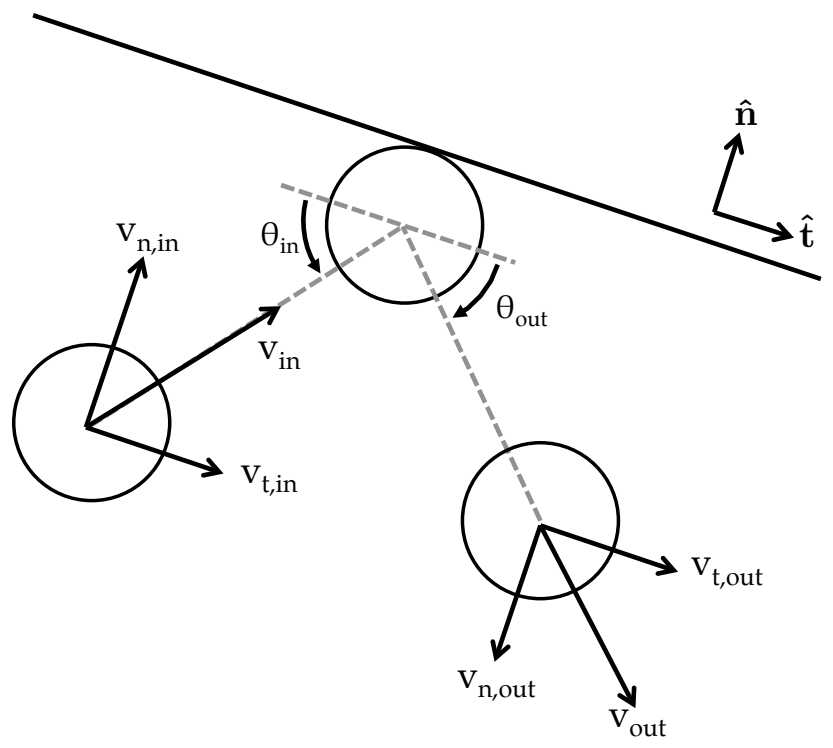

Figure 6.3: Diagram of particle bouncing

It should be possible to calculate $C O R_{\hat{n}}$ from the MP-PIV data by extracting the velocity of particles close to the wall; Fig. 6.4 shows the line that was selected. Along this line, each instantaneous MP-PIV vector was decomposed into wall-normal and wall-tangent vectors. Each vector was then sorted into either an incoming $\left(\vec{v}_{i n} \cdot \hat{n}>0\right)$ or outgoing $\left(\vec{v}_{i n} \cdot \hat{n}<0\right)$ particle. The trajectory of each particle is linearly extrapolated towards the wall (forward for incoming particles, backwards for outgoing particles) to calculate is impact location. Because 


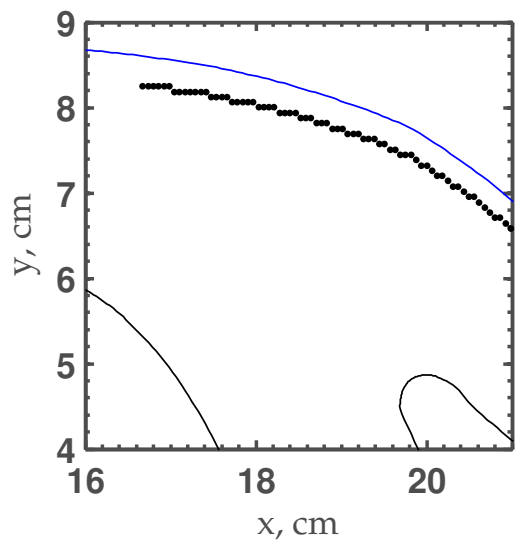

Figure 6.4: Restitution calculation location.

the MP-PIV is incapable of tracking a single particle, the results are ensemble averaged to determine the $C O R_{\hat{n}}$. This techniques is similar to the one employed by Reagle et al. ${ }^{[36,37]}$.

\subsection{Results}

\subsubsection{OSG-1}

MP-PIV results for the $10 \mu \mathrm{m}$ glass spheres for the baseline case of OSG-1 at the design flow split of $\beta=0.16$ are shown in Fig. 6.5. Figure 6.5a shows the mean particle velocity profile with overlaid particle pathlines *. At the entrance to the bifurcation region $(x=17 \mathrm{~cm})$, the path-lines are concentrated near $y=7 \mathrm{~cm}$. This tight grouping is created by the outward then inward turns that occur upstream, as shown in the data from Fig. 6.1. Following these pathlines through the bifurcation region, most enter the scavenge flow leg, indicating that particles are being separated. There are also several lines that enter the core flow, showing the

*MP-PIV actually measures the streamlines of the particle velocity field, not the pathlines of the particles themselves, which would be ideal. However, pathlines and streamlines are equivalent once the data are averaged. Therefore, pathlines are used in this text in reference to the particle velocity field and streamlines to refer to the fluid velocity field. 
inefficiency of OSG-1 for the $10 \mu \mathrm{m}$ particles. Another noticeable aspect is that there were no usable vectors in the core leg on the hub side, which means that the IPS is functioning properly and the inertia of the particles is sending them (mostly) into the scavenge leg. Along the wall there are some vectors that appear with near zero velocity, which is a result of laser reflections on the hub geometry.

Fig. 6.5b shows the number of usable vectors determined at each point. Because these vectors can only be calculated where particles are present, the vector count can be qualitatively correlated with the average concentration of particles in the IPS. This figure shows a narrow stream of incoming particles at $x=17 \mathrm{~cm}$ that is dispersed as it approaches the scavenge flow path and partially enters the core flow leg. These pathlines do not agree with those given by Hamed et al. for $10 \mu \mathrm{m}$ sand particles, but are correlated to the $3 \mu \mathrm{m}$ particle traces. This may be caused by the small particles present in the $10 \mu m$ particle set. Along the OSG there are high concentration of particles where flow separation was identified in oil-streak measurements ${ }^{[5]}$. This high concentration of particles is evidence of entrainment in the flow recirculation.

Figures $6.5 \mathrm{c}$ and $6.5 \mathrm{~d}$ show the standard deviation and the standard deviation normalized by the mean velocity, respectively. Particles enter the measurement region with consistent velocity, but display a large variation as soon as they approach the splitter geometry and enter the core and scavenge flow legs. Relative to their mean values, the standard deviation of the particle velocity in the scavenge region is more than $50 \%$ and $100 \%$ in the separated flow region.

Figure 6.6 shows similar results using the $35 \mu \mathrm{m}$ particles. The $35 \mu \mathrm{m}$ particles have more inertia, carrying them to the outside of the turn and causing them to mainly stay much closer to the OSG than the $10 \mu \mathrm{m}$ particles. The $35 \mu \mathrm{m}$ particles are not attracted to the separation region as much as the $10 \mu \mathrm{m}$ particles, but rather show a more uniform 


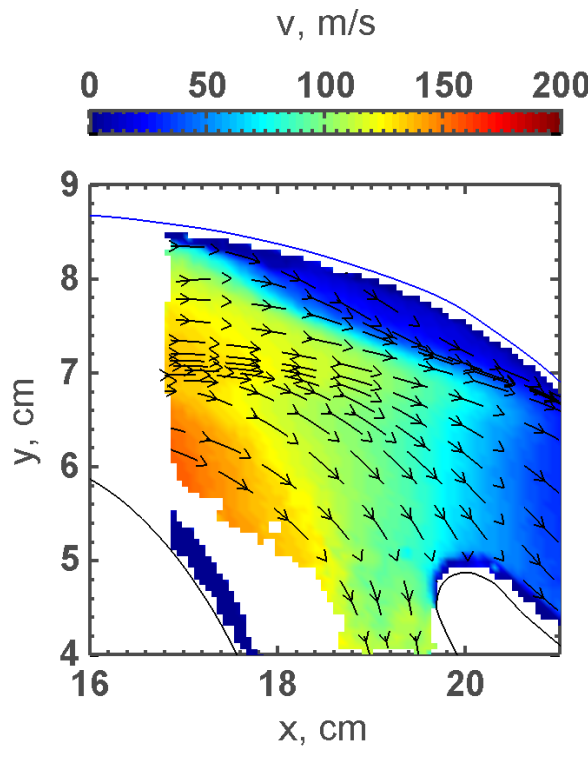

(a) Velocity Magnitude

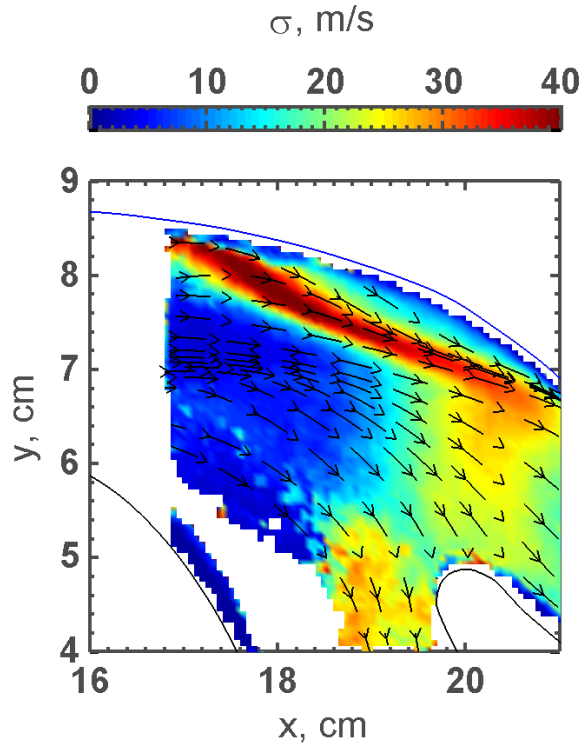

(c) Standard Deviation

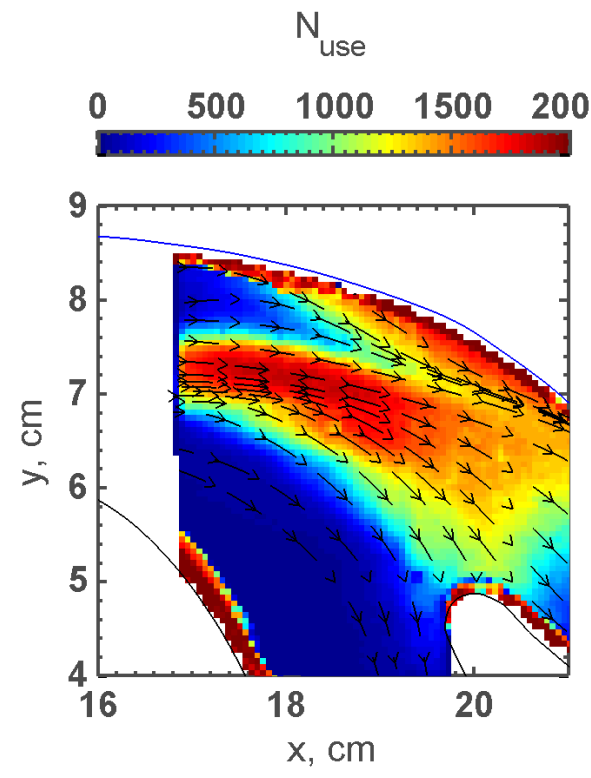

(b) Usable Vectors

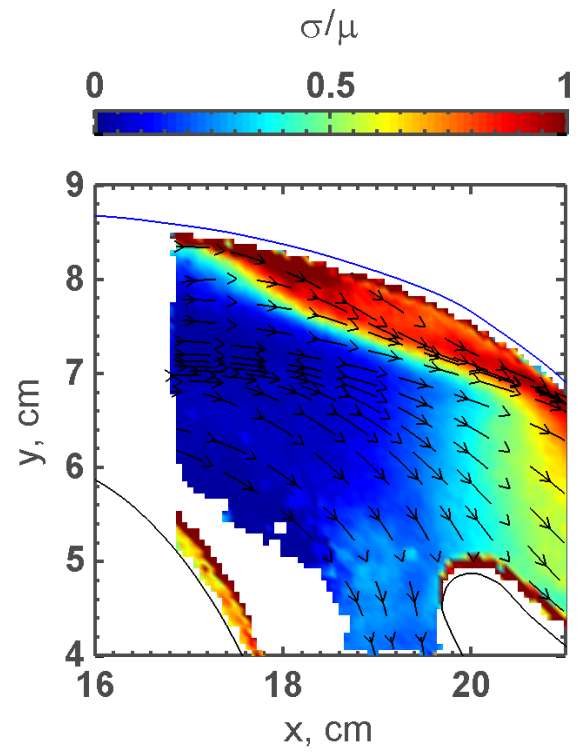

(d) Normalized Standard Deviation

Figure 6.5: Average quantities of $10 \mu \mathrm{m}$ particles for OSG-1 
distribution along the OSG as a result of IPS operation. Again, the particles inertia carries them through the IPS with less influence from the fluid forces.

However, as in the case of the $10 \mu \mathrm{m}$ particles, the correlation between the general particle behavior predicted by Hamed et al. ${ }^{[11]}$ and that observed for the $35 \mu \mathrm{m}$ particle is not fully observed. Rather, a significant number of apparent particle paths deviate from the predicted behavior near the splitter, with a portion of those particulate entering the core and causing separator inefficiency.

Figure 6.7 shows the particle pathlines for the $10 \mu \mathrm{m}$ and $35 \mu \mathrm{m}$ particles compared with the gas flow streamlines from Barone et. al ${ }^{[32]}$. The $10 \mu m$ particles come into the bifurcation region with nearly flat (no velocity component in the y direct) pathlines and do not follow the fluid streamlines that are already turning downward. By $x=19 \mathrm{~cm}$, the particle pathlines in the core are significantly correlated with the fluid streamlines and particle pathlines entering the scavenge leg show the effects of the fluid turning. By $x=20 \mathrm{~cm}$, particles near the splitter nose are following the streamlines, but particles near the OSG are not. This region is characterized by high deviation in both the fluid and particle velocities, resulting in the pathlines that do not correlate to the streamlines. Comparing to the high-speed video from Barone et al. ${ }^{[32]}$, this region cannot be adequately explained using the average flow field due to these high instabilities that are present.

The $35 \mu \mathrm{m}$ particles (Fig. 6.7b) appear to enter the bifurcation region in two different regimes relative to the fluid flow: 1) particles enter near $y=7 \mathrm{~cm}$ and are concentrated by the two upstream turns similar to the $10 \mu \mathrm{m}$ particles and 2) particles enter with a slight downward velocity indicating they have just bounced off of the OSG, upstream of the cameras field of view. These two streams merge around $x=19 \mathrm{~cm}$ and the pathlines become convoluted thereafter, making it difficult to distinguish paths of these particles. The final pathline that exists at the splitter nose directly at the bifurcation point. Due to the nature 


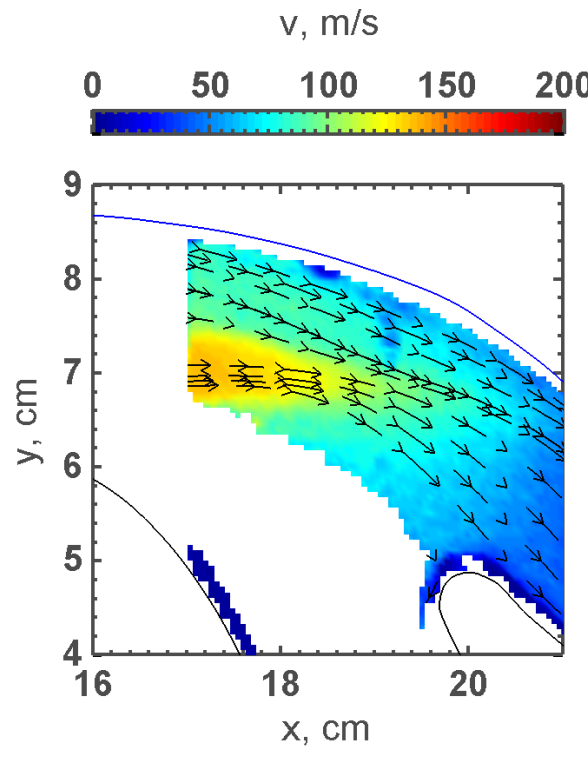

(a) Velocity Magnitude

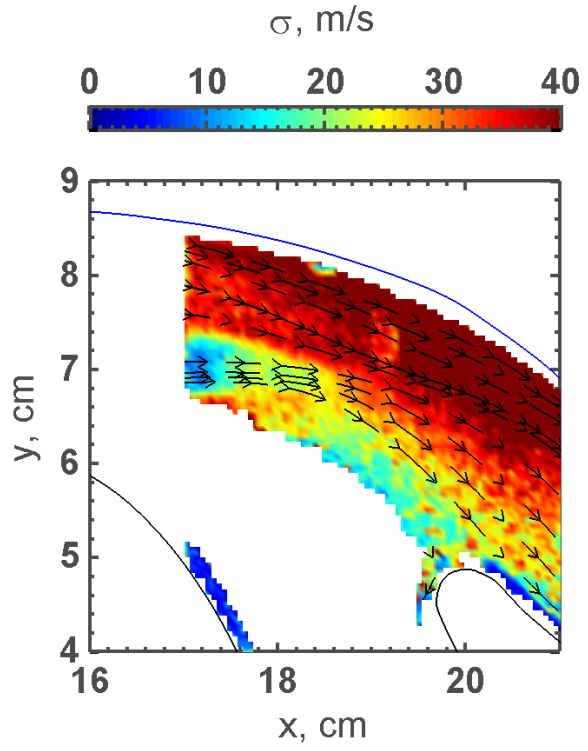

(c) Standard Deviation

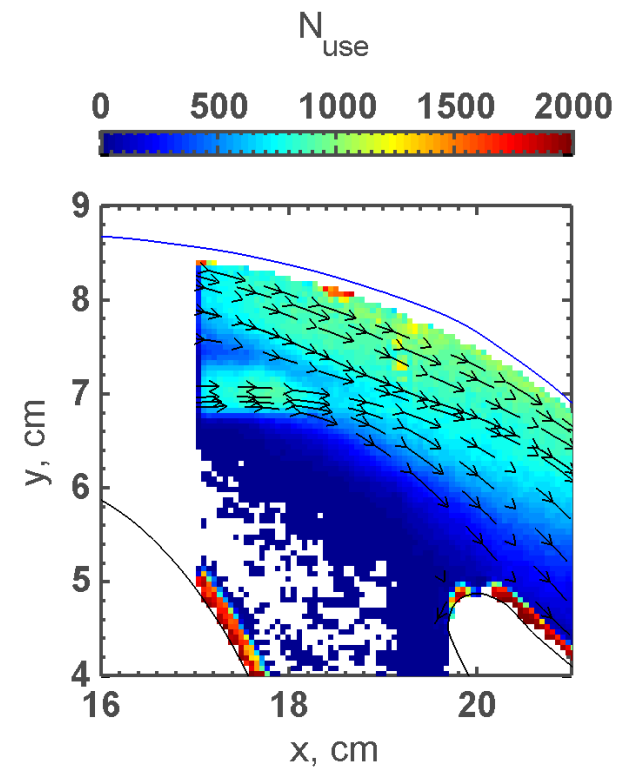

(b) Usable Vectors

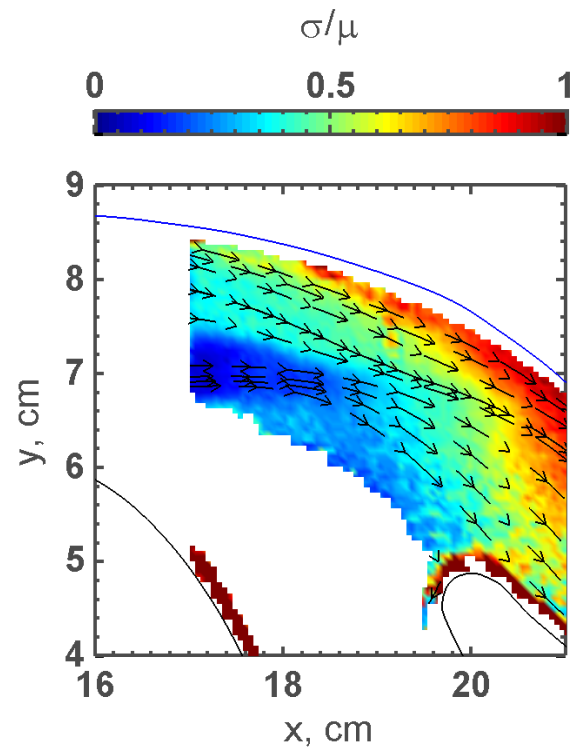

(d) Normalized Standard Deviation

Figure 6.6: Average quantities of $35 \mu \mathrm{m}$ particles for OSG-1 


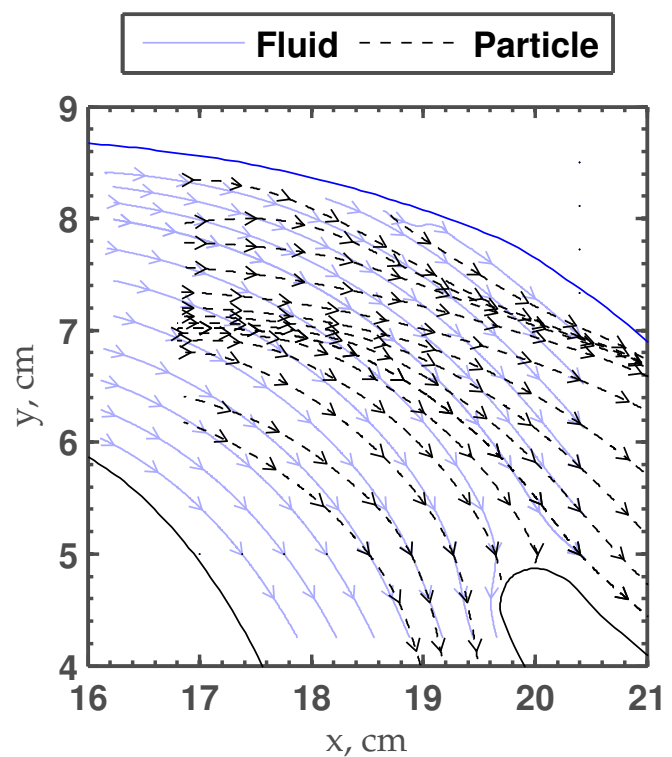

(a) $10 \mu m$ Particles

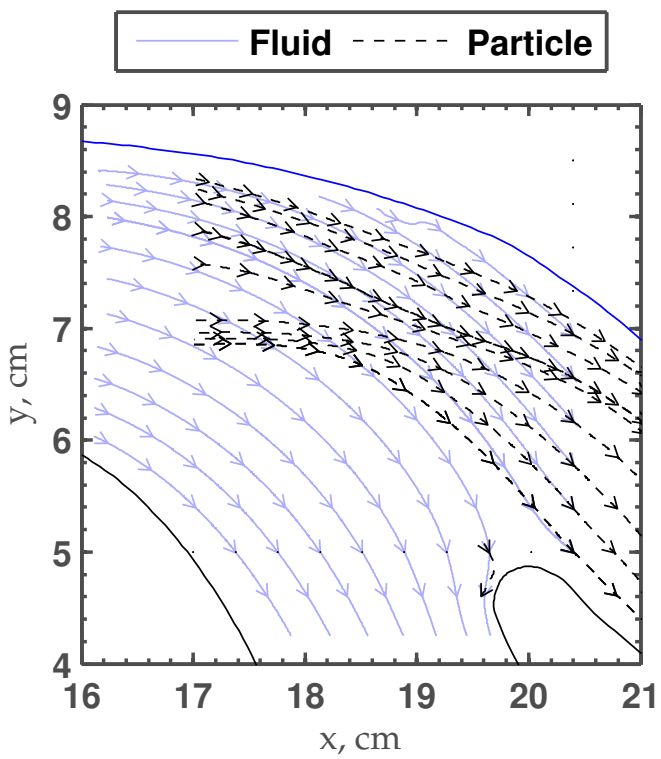

(b) $35 \mu m$ Particles

Figure 6.7: Particle trace to streamline comparison for OSG-1

of the PIV imaging, no data exists for this pathline upstream, but it is apparent that some particles are being ingested into the core leg. The lack of data upstream and the high-speed video for $10 \mu \mathrm{m}$ particles suggests that these particles are most likely coming around the splitter nose from the scavenge leg.

\subsubsection{OSG-2}

Figure 6.8 and 6.9 show the PIV results of $10 \mu \mathrm{m}$ and $35 \mu \mathrm{m}$ particles, respectively, for OSG-2. In both cases, the particles still enter the bifurcation region in a stream at $y=7 \mathrm{~cm}$. As a recirculation zone is present in the particle pathlines, particles are being entrained, similar to those indicated by Hamed et al. In Fig. 6.8b, the usable vectors show a large concentration of particles in the recirculation zone. Particles are being constantly recirculated, which could impact separation efficiency if they are pulled into the core by some mechanism. 
The standard deviation and normalized standard deviation are omitted from current and remaining figures because their content is very similar to those in Figs. $6.5 \mathrm{c}$ and $6.5 \mathrm{~d}$.

It is again noted that a portion of the $10 \mu \mathrm{m}$ and $35 \mu \mathrm{m}$ particulate deviate from the expected particle path and pass more closely to the splitter than expected, with a portion of the particles entering the core. This effect is generally stinger for the OSG-2 geometry than for OSG-1. This agrees with the overall efficiency data trends in Tbl. 6.1.

Figure 6.10 compares the particle MP-PIV with the gas flow PIV results for OSG-2. The $10 \mu \mathrm{m}$ particles (Fig. 6.10a) entering the bifurcation act in a similar manner to the $10 \mu \mathrm{m}$ particles in OSG-1. Initially, the particle pathlines do not follow the fluid streamlines, but when the pathlines exit the interrogated region, they have nearly matched the streamlines. Also, the particles present in the recirculation zone nearly track the fluid. Furthermore, the particle pathline that turns into the core near the splitter nose crosses a fluid streamline twice, appearing to work against the prevailing flow! The only possible explanation for this type of behavior is flow unsteadiness around the splitter nose (e.g. events like flow reversal in the scavenge leg are causing this behavior in the pathlines). This effect is even more pronounced in Fig. 6.10b for the $35 \mu \mathrm{m}$ particles. The $35 \mu \mathrm{m}$ particles, similar to OSG-1, show highly inertia-dominated paths, with some fluid effects. These fluid effects are most notable in the recirculation zone, where particles follow the flow streamlines near the OSG.

\subsubsection{OSG-3}

Figure 6.11 and 6.12 show the MP-PIV results of $10 \mu \mathrm{m}$ and $35 \mu \mathrm{m}$ particles, respectively, for OSG-3. As with OSG-1 and OSG-2 the particles still enter the bifurcation region in a stream at $y=7 \mathrm{~cm}$, demonstrating consistency of the particles as they enter the bifurcation region. The $10 \mu m$ particles are affected by the flow more than the $35 \mu m$ particles and 


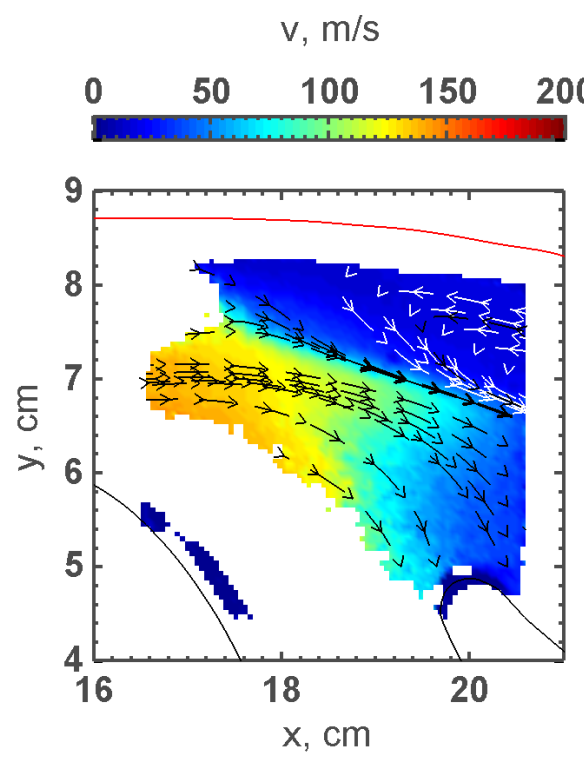

(a) Velocity Magnitude

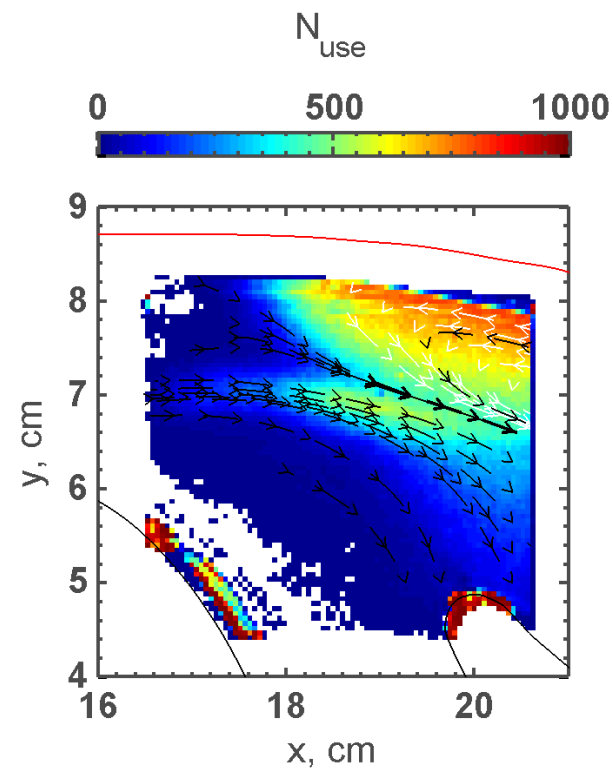

(b) Usable Vectors

Figure 6.8: Average quantities of $10 \mu m$ particles for OSG-2

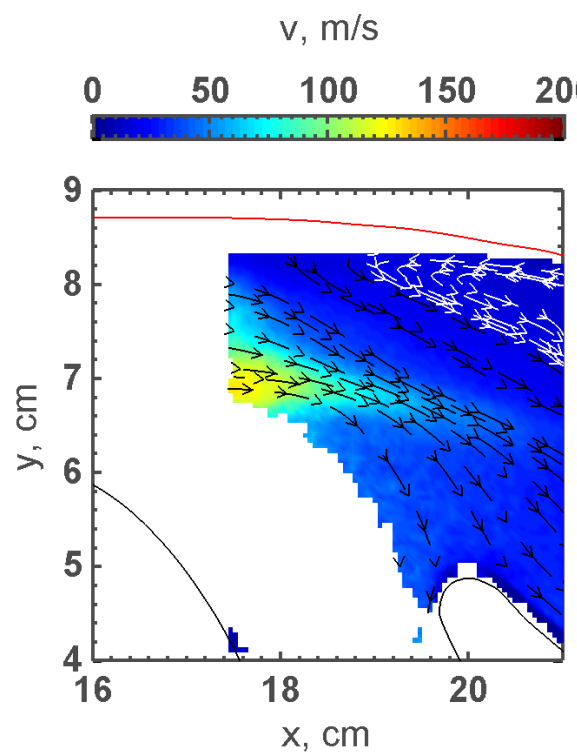

(a) Velocity Magnitude

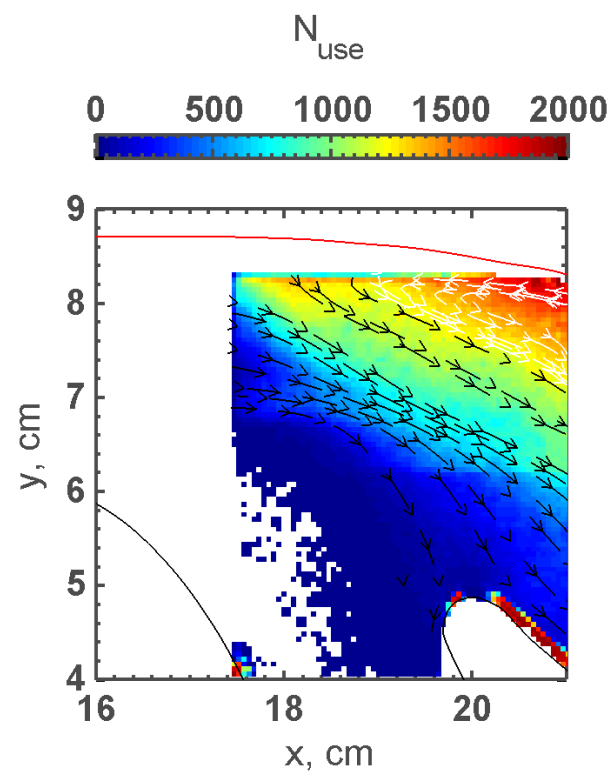

(b) Usable Vectors

Figure 6.9: Average quantities of $35 \mu \mathrm{m}$ particles for OSG-2 


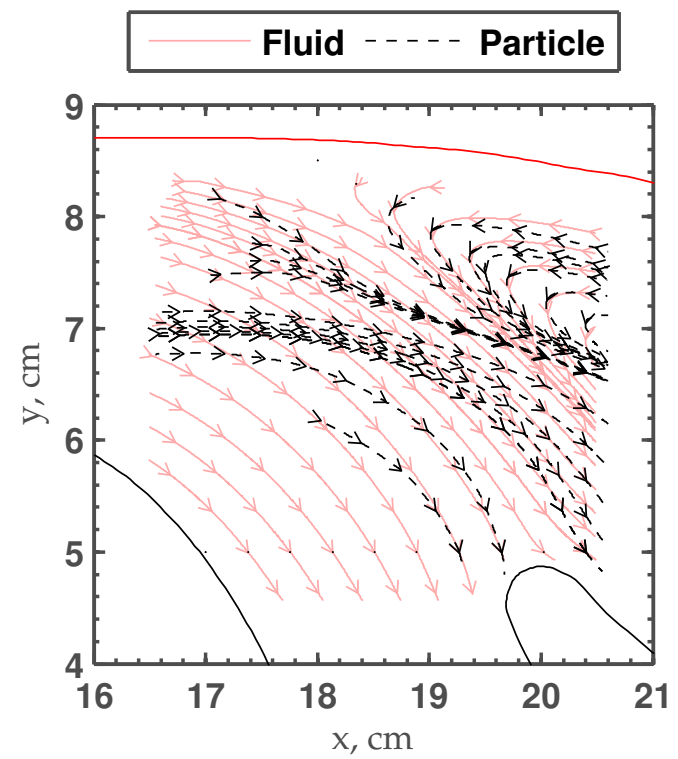

(a) $10 \mu m$ Particles

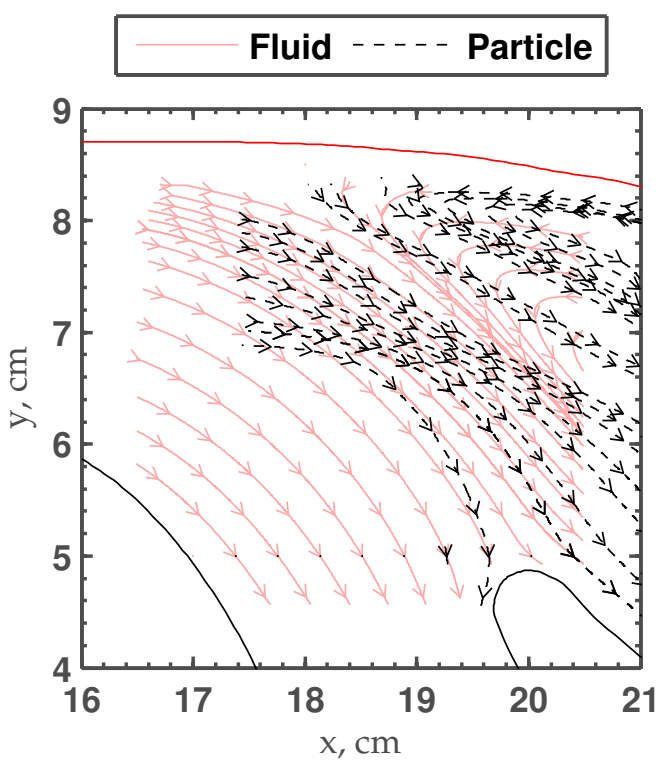

(b) $35 \mu m$ Particles

Figure 6.10: Particle trace to streamline comparison for OSG-2

the average recirculation zone is larger for the $10 \mu \mathrm{m}$ particles than the $35 \mu \mathrm{m}$ particles corresponding to the larger inertia of the $35 \mu \mathrm{m}$ particles. In these figures, it does appear that OSG-3 is less efficient at separating particles than OSG-1 and OSG-2, because there are more particle pathlines entering the core flow. This is not the case, however, as can be seen by examining the data in Tbl. 6.1. The differences can be attributed to favorable experimental conditions that allowed those regions to be captured by the camera with more detail. There are two possible explanations for this effect: 1) the shape of OSG-3 is favorable to the laser sheet entering the test section, due to lensing effects as the laser sheet passes through the tape; and 2) that the improved visibility is caused by slight shifts in the laser-camera setup. The PIV setup is often moved between experiments so the OSG can be interchanged. While every effort is put forth to have consistent experimental conditions, small variations exist between runs. 


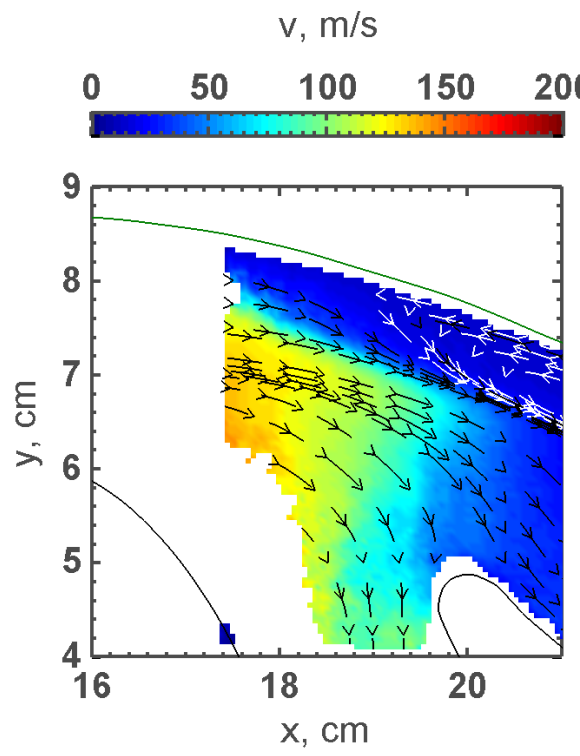

(a) Velocity Magnitude

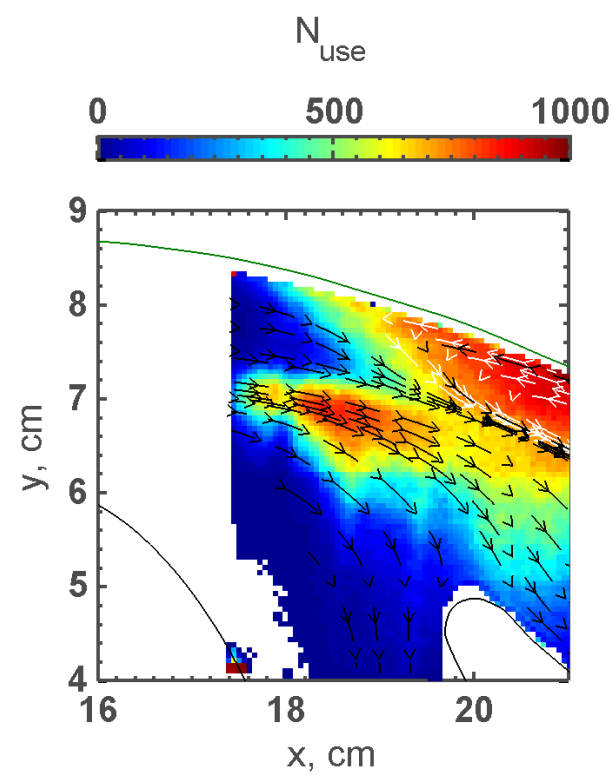

(b) Usable Vectors

Figure 6.11: Average quantities of $10 \mu \mathrm{m}$ particles for OSG-3

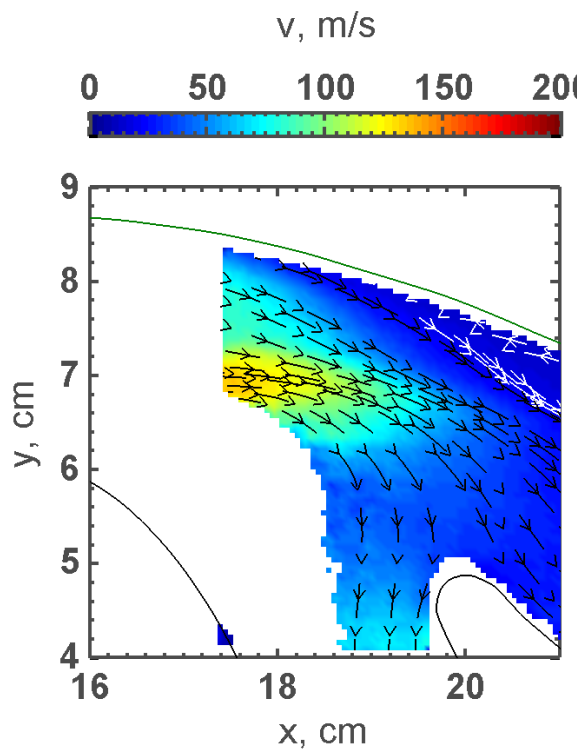

(a) Velocity Magnitude

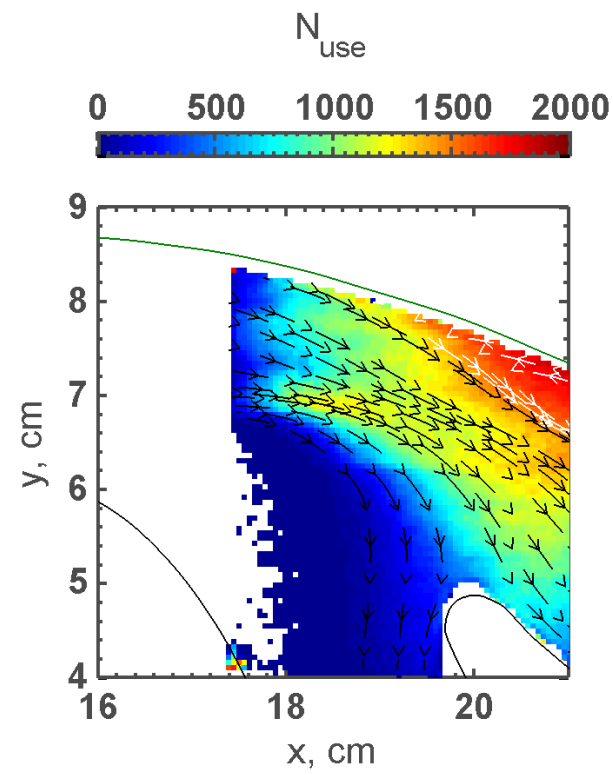

(b) Usable Vectors

Figure 6.12: Average quantities of $35 \mu \mathrm{m}$ particles for OSG-3 


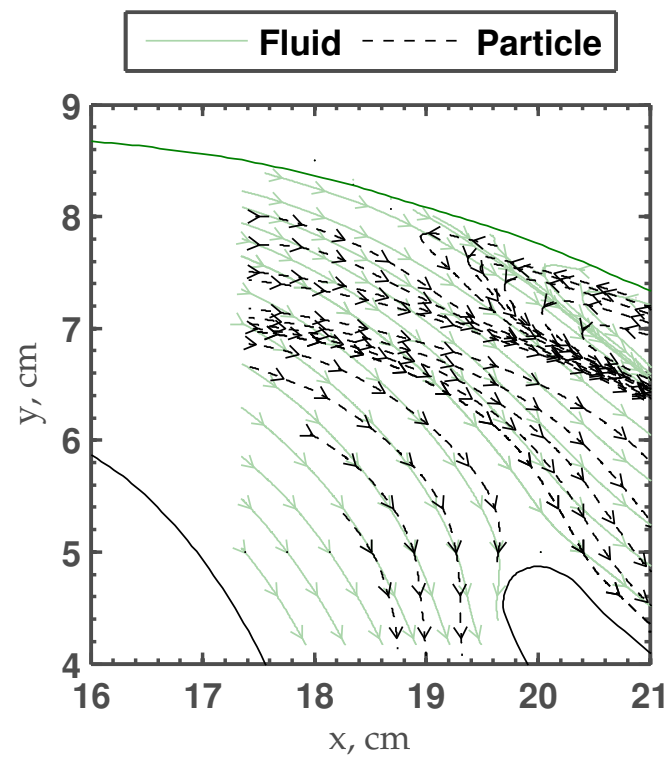

(a) $10 \mu m$ Particles

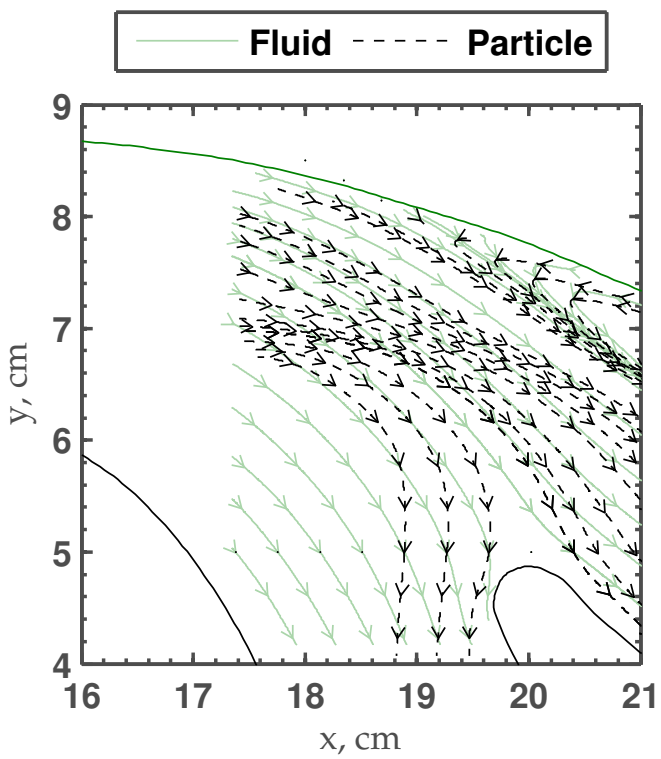

(b) $35 \mu m$ Particles

Figure 6.13: Particle trace to streamline comparison for OSG-3

Figure 6.13 compares the particle pathlines for OSG-3 to the fluid streamlines. The same effects for both the $10 \mu \mathrm{m}$ and $35 \mu \mathrm{m}$ particles are seen in this figure as in Figs. 6.7 and6.10 for OSG-1 and OSG-2. Similar to OSG-2 there are pathlines that cross over the same fluid streamline more than once and appear to work against the average fluid streamlines. This case shows the pattern clearly where the pathlines enter the core flow leg for the $35 \mu \mathrm{m}$ particles. The pathlines in the recirculation zones of the $10 \mu \mathrm{m}$ and $35 \mu \mathrm{m}$ particles overshoot the fluid recirculation zone. This is caused by particles being accelerated along the wall and their inertia carrying them forward, past the fluid recirculation zone. This was not seen in OSG-2 where the recirculation zone is larger and slower. 


\subsubsection{Particle Restitution}

Utilizing the data from the glass particle PIV for OSG-1, it is possible to estimate an ensembleaveraged $C O R_{\hat{n}}$ for the spheres as they impact and reflect off of the OSG. Taking the ratio of the outbound to the inbound particle velocities results in the $C O R_{\hat{n}}$ in Fig. 6.14a. Of note, there is one point with a $C O R_{\hat{n}}$ larger than one, which can be attributed to uncertainty and bias in the measurement technique. Figure $6.14 \mathrm{~b}$ shows the impact angle of particles along the OSG. The impact angle is consistently between 10 and 20 degrees. This shallow angle means that particles are not coming straight across the bifurcation region and hitting the OSG, as the OSG angle is much greater (ranging 10 to 50 degrees from $x=16 \mathrm{~cm}$ to $x=21 \mathrm{~cm})$.

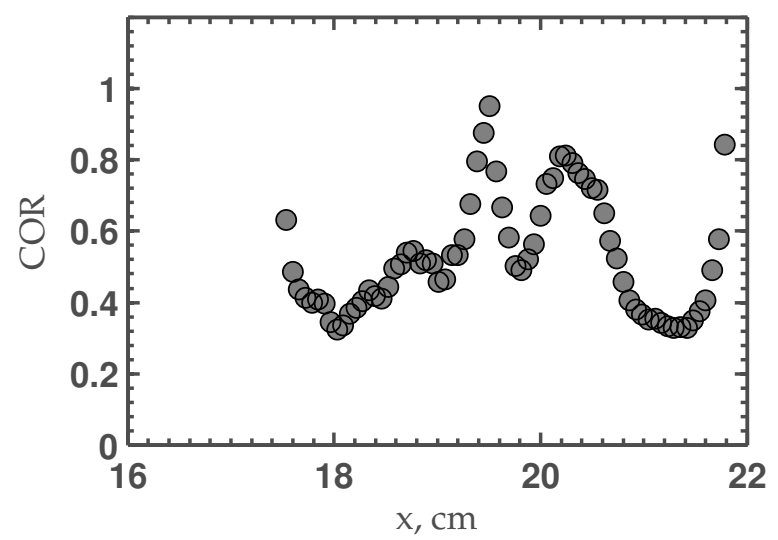

(a)

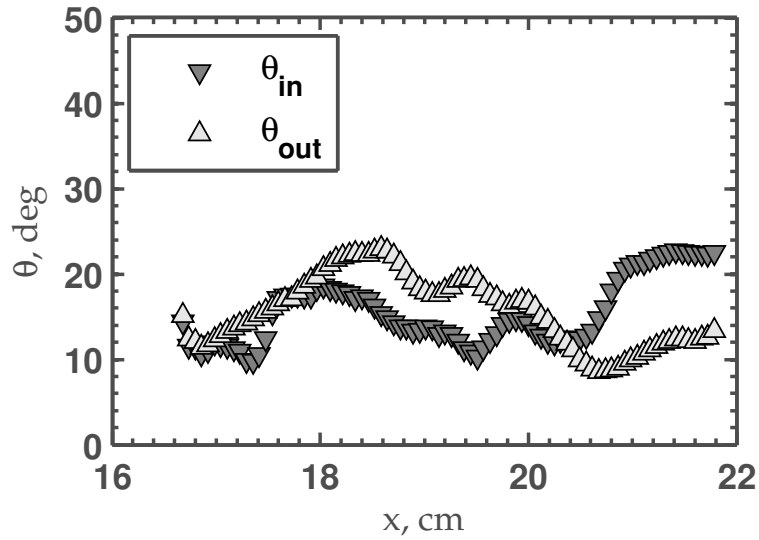

(b)

Figure 6.14: Coefficient of Restitution for $10 \mu \mathrm{m}$ glass spheres as a function of location

Figure 6.15 shows the instantaneous and averaged tangential velocity components for $10 \mu m$ particles measured near the OSG. The mean velocities are filtered to reduce noise that arrises in the $C O R_{\hat{n}}$ measurement. A 5-point moving average is applied to both the incoming and outgoing mean velocity curves. The mean incoming velocity varies between $5 \mathrm{~m} / \mathrm{s}$ and $25 \mathrm{~m} / \mathrm{s}$ and the outgoing velocity varies from $4 \mathrm{~m} / \mathrm{s}$ to $7 \mathrm{~m} / \mathrm{s}$. There is also 
a distinct $20 \mathrm{~m} / \mathrm{s}$ gap in the incoming velocities, particularly between $x=17.5 \mathrm{~cm}$ and $x=19 \mathrm{~cm}$. The particles with higher velocity may be particles that have just entered the IPS while the lower velocity particles may be those entrained in a recirculation event or particles than have already bounced multiple times. It is difficult to determine exactly how the particles are bouncing through the OSG without clearer time correlated data.

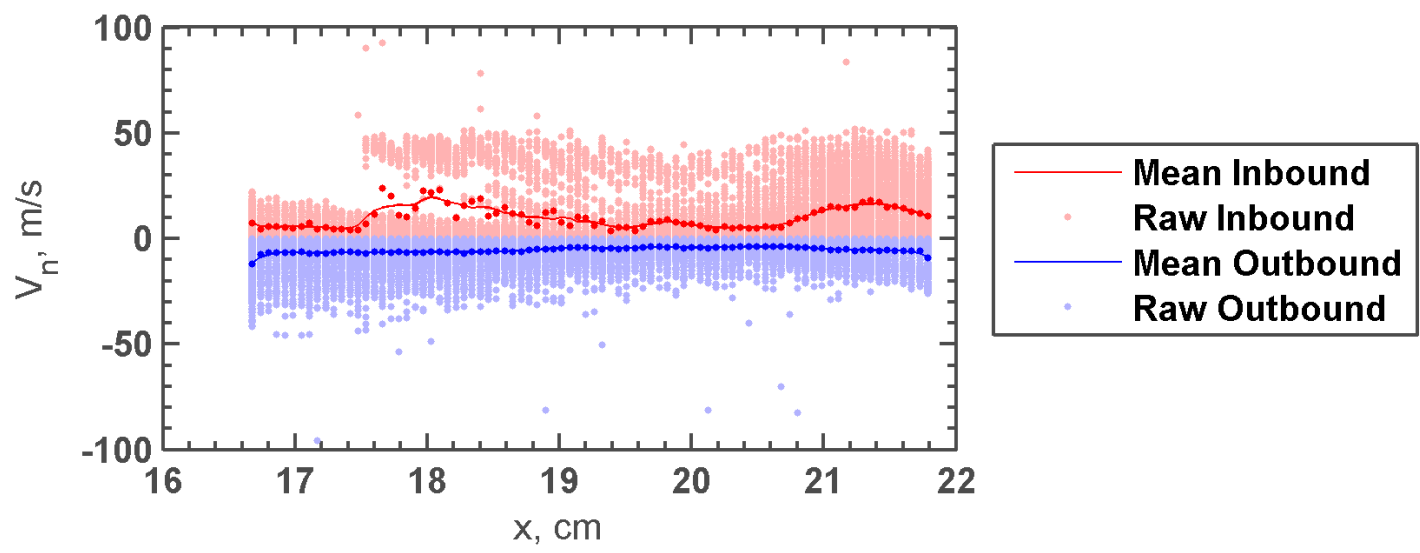

Figure 6.15: Inbound and outbound wall normal velocity of $10 \mu \mathrm{m}$ particles. The mean velocities are filtered using a 5-point moving average to reduce noise in the $C O R_{\hat{n}}$ measurement.

It is difficult to determine exactly how the particles are bouncing through the OSG without clearer time correlated data. The MP-PIV produces excellent particle definition without time correlation, and the high-speed video provides time correlation without clear particle definition. Figure 6.16 provides three different particle pathlines that fit the observed data from MP-PIV and the high-speed video.

In Fig. 6.17, the $C O R_{\hat{n}}$ is plotted by velocity and compared to the data and theory from Singh and Tafti ${ }^{[38]}$ for $10-20 \mu m$ A4 dust and theory from Jackson et al. ${ }^{[39]}$. The dust should have the same properties to that of A4 due to the common silica composition and because the particles are modeled spheres. Particles within the IPS impact the walls at a wide range of velocities and the $C O R_{\hat{n}}$ can vary significantly depending on the speed at which it collides with the wall. This change in the $C O R_{\hat{n}}$ at high velocity is caused by the viscoelastic effects 


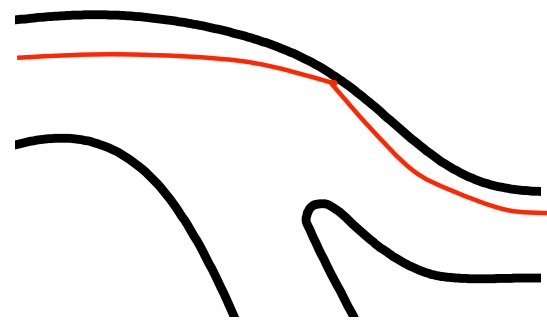

(a)

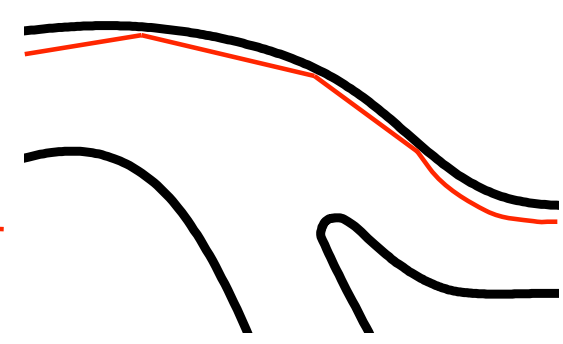

(b)

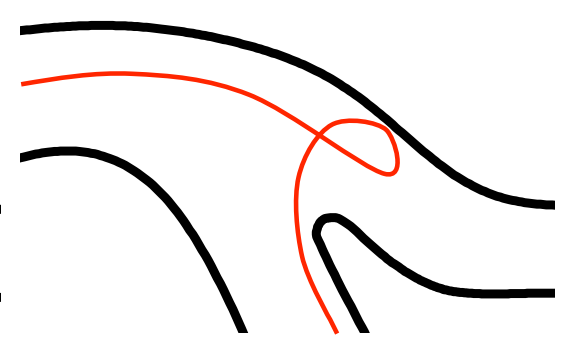

(c)

Figure 6.16: Schematic of three possible $10 \mu \mathrm{m}$ particle pathlines that match the small impact angles and high-speed video: a) Incoming high-speed particle path weakly affected by fluid forces, b) a particle bouncing multiple times across the OSG at shallow angles, and c) a slow particle that encounters an ingestion event.

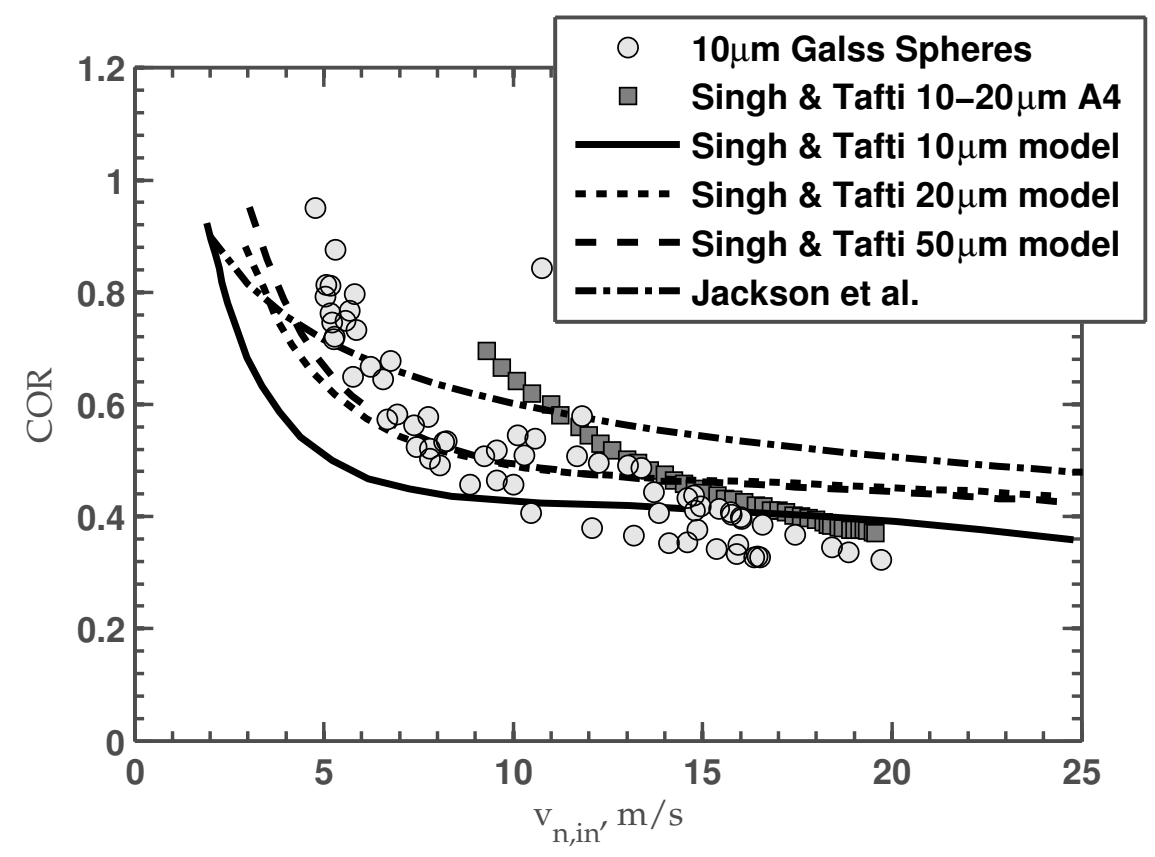

Figure 6.17: Coefficient of Restitution for $10 \mu \mathrm{m}$ glass spheres as a function of incoming perpendicular velocity

within the particle and the wall. As the velocity of the particle increases these effects become greater, reducing the $C O R_{\hat{n}}$, relative to that at low speeds.

The same process was completed for the $35 \mu \mathrm{m}$ particle data. The $C O R_{\hat{n}}$ is shown in Fig. 6.18 a, with ranges of 0.3 to 0.8 and one point with a $C O R_{\hat{n}}$ near one. The impact angle 


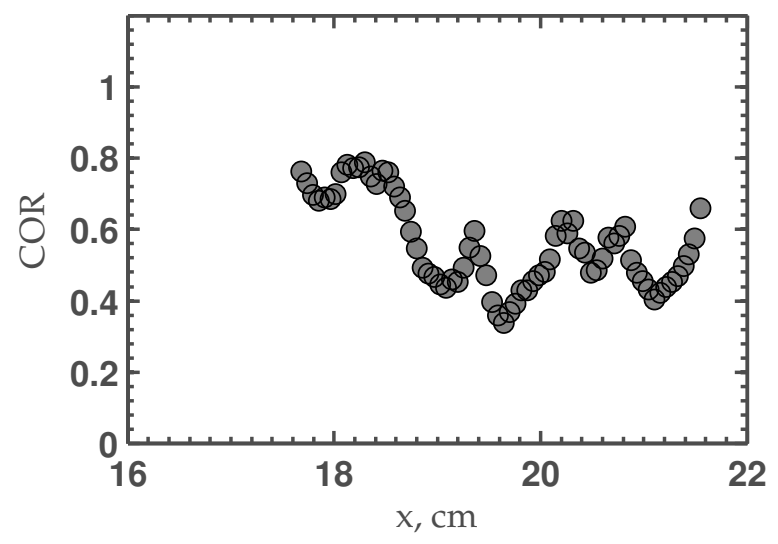

(a)

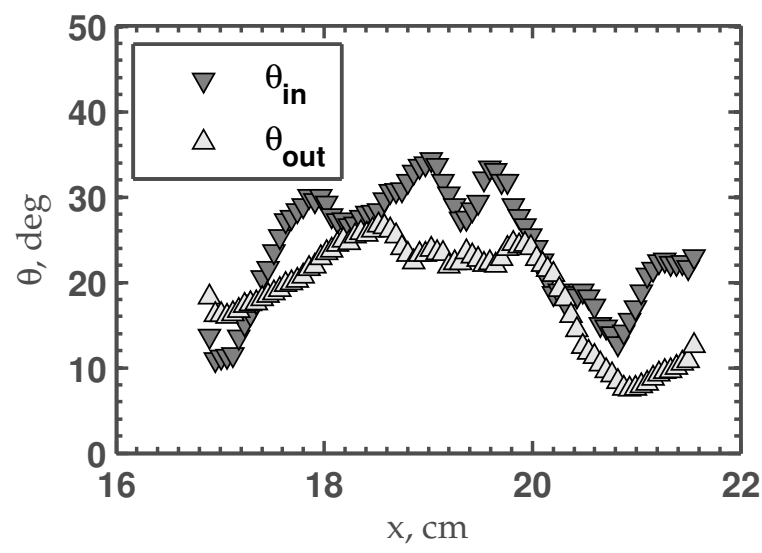

(b)

Figure 6.18: Coefficient of Restitution for $35 \mu \mathrm{m}$ glass spheres as a function of location

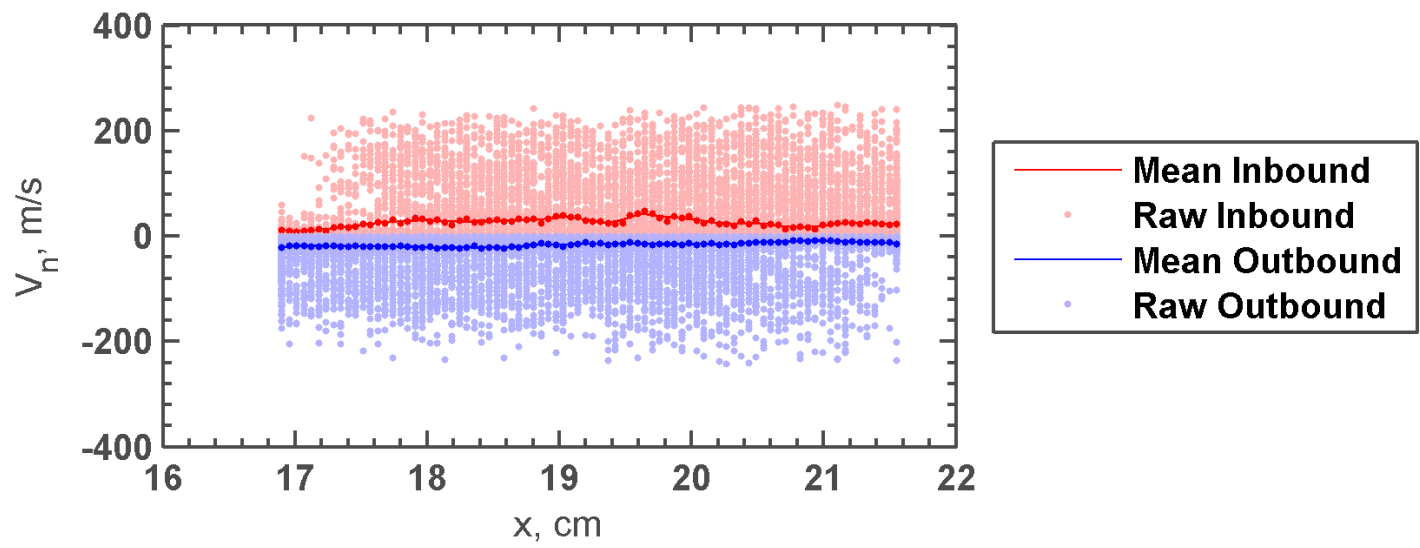

Figure 6.19: Inbound and outbound wall normal velocity of $35 \mu \mathrm{m}$ particles. The mean velocities are filtered using a 5-point moving average to reduce noise in the $C O R_{\hat{n}}$ measurement.

in Fig. 6.18b varies from 10 to 30 degrees along the wall. These impact angles correspond to the angle of the wall of 15 to 30 degrees between $x=18 \mathrm{~cm}$ to $x=20 \mathrm{~cm}$. This indicates that the $35 \mu \mathrm{m}$ particles are traversing straight across the bifurcation region and impacting along the OSG and that minimal fluid forces are affecting the particles. Figure 6.19 shows the inbound and outbound particle velocities. The mean inbound normal velocity varies from $5 \mathrm{~m} / \mathrm{s}$ to $25 \mathrm{~m} / \mathrm{s}$ and the mean outbound velocity is between $4 \mathrm{~m} / \mathrm{s}$ and $7 \mathrm{~m} / \mathrm{s}$. 


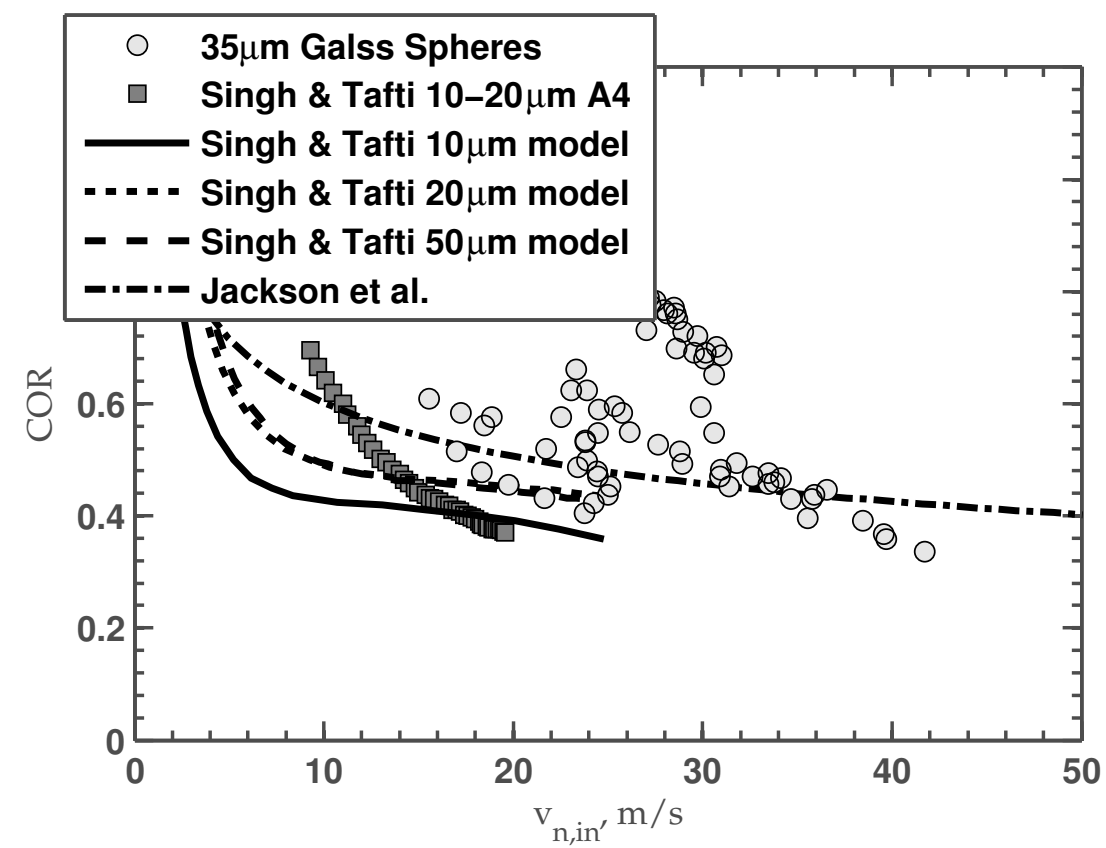

Figure 6.20: Coefficient of Restitution for $35 \mu \mathrm{m}$ glass spheres as a function of incoming perpendicular velocity

Figure 6.20 shows the data plotted by the incoming velocity compared with the same data and theory from Singh and Tafti ${ }^{[38]}$. Upstream of the recirculation zone $(x<19.3 \mathrm{~cm})$, the $35 \mu m$ particles enter in at much higher velocities than the $10 \mu m$ particles. The glass spheres match the particle bounce theory reasonably well, although there is a large amount of scatter in the data. The scatter in this data (and the $10 \mu m$ data) is due to the difficulty in determining the $C O R_{\hat{n}}$ of particles. Adding the complexities of dynamic fluid forces, ensemble averaging, and measurement difficulties near the wall, makes the measurement that much more difficult. 


\subsection{Conclusions}

Muliti-Phase PIV measurements for $10 \mu m$ and $35 \mu m$ particles were successfully completed for three IPS geometries in the wind tunnel at the University of Virginia. The particle paths are characterized by steady inflow of particles into the bifurcation zone as a result of the upstream turns. This action allows most of the particles to enter the scavenge leg demonstrating successful particle separation. However, some particles are ingested into the core flow, resulting in inefficient operation. Both the $10 \mu \mathrm{m}$ and $35 \mu \mathrm{m}$ particles also become entrained in the recirculation zone, which appears to give them a greater chance to be transferred into the core. Overall, the $35 \mu \mathrm{m}$ particle pathlines show more evidence of inertial motion than the $10 \mu m$ particles, giving them higher separation efficiency, but they are still affected by some mechanism causing them to deviate towards the core.

The $C O R_{\hat{n}}$ for particles impacting the OSG near the recirculation zone varied with the incoming velocity. Particles are entering at high enough velocities that inelastic particle-wall collisions are occurring. These collisions match with well with previous experiments and theory. Based on the impact angle, the $35 \mu \mathrm{m}$ particles that impact the wall appear to come straight across the bifurcation region. However, the pathlines of the $10 \mu \mathrm{m}$ particles are not as straightforward; as the smaller particles are influenced by fluid forces to a much greater extent than the larger ones as expected.

The fluid unsteadiness in the scavenge region is affecting the particles, resulting in a loss of separation efficiency. The previous computational work in IPS design has focused significantly on particle bounce and drag models, which appear to be reasonable for the mean flow. However, this study shows that using the mean flow is insufficient to capture the dynamic effects of the fluid on the particles that result in a loss of possible separation efficiency. 
CHAPTER 7

\section{Conclusions}

This project successfully discovered the dynamic multiphase interactions present in inertial particle separators. All of the objectives set out at the beginning have been met, the goal accomplished.

\subsection{Objective 1: Facility Design}

A facility has been designed and built to study the complex flows associated with inertial particle separators. The tunnel reproduces the mass flow and particle conditions that are seen in IPS systems. The tunnel is capable of independent flow leg control enabling studies to explore variations in the total mass flow and scavenge split. The PDS is capable of delivering sand, dust, and other particulate into the flow field to mimic a variety of conditions an IPS may encounter. The test section has been developed in order to explore many OSGs and their effects on efficiency. Oil-streak measurements of the test section show a substantial region of 
2-dimensional flow with areas near the walls demonstrating significant 3-dimensional regions for further study.

\subsection{Objective 2: Efficiency Measurements}

There is a significant correlation between the position of the recirculation zone and the efficiency of the IPS geometry. This finding supports the hypothesis that the recirculation itself has a significant impact on separation efficiency. The separation efficiency of an IPS is also dependent on the scavenge flow split, whereby increasing the amount of scavenge flow results in an improvement in efficiency.

The efficiency of $10 \mu \mathrm{m}, 35 \mu \mathrm{m}$ and $120 \mu \mathrm{m}$ glass spheres were also measured in the three OSGs. The results confirm the dependence of efficiency on particle size, with the larger particles having higher separation efficiencies than the smaller particles. Based on this data, a model was developed that further explained the decrease in separation efficiency based on particle Stokes number. In agreement with the model, the $120 \mu \mathrm{m}$ particles were completely separated (within experimental error) and the smaller $35 \mu \mathrm{m}$ particles saw less efficient separation due to the influence of fluid dynamic forces. The $10 \mu \mathrm{m}$ particles, however, were over predicted by the model, which may provide a link to the previous postulation that there are unsteady effects in the bifurcation region, thus hindering separation efficiency. Effects in the bifurcation region would be more pronounced with the smallest particles, as they have less inertia and are significantly influenced by the fluid forces acting on them. The use of several sizes of narrowly dispersed particles that have constant shape and density also provide an ideal comparison for future computational studies. 


\subsection{Objective 3: Airflow and Particle Velocity Fields}

PIV measurements conducted in the research facility have shown that there are significant dynamic flow structures occurring within the IPS. The average flow fields for OSG-2 and OSG-3 match the previous oil-streak data for these geometries. OSG-1 does not indicate recirculation in the mean for the PIV field of view. However, there is recirculation in the instantaneous flow fields indicating incipient separation. Each OSG has a region of unsteady flux at the entrance to the scavenge flow leg in conjunction with a shear zone as indicated by the streamwise vorticity. This unsteadiness in the flow rate appears to cause the recirculation zone to be intermittent, especially for OSG-1.

Two-dimensional mass flux analysis of the bifurcation region showed instantaneous net gains and losses during IPS operation, explained byintermittent span-wise flux occurring during IPS operation. This was evident from the instantaneous results of the flow fields for all three OSGs, further showing the dynamic nature of the scavenge flow leg. The instantaneous velocity fields indicate two periods of normal operation (resembling the mean flow), periods where the scavenge leg is dominated by high velocity flow, and periods where the flow in the scavenge leg is reversed and ingested into the core flow leg. Each of the OSGs displayed the same intermittent behaviors, but each one had different characteristics in the scavenge flux plane. These differences correlate to the size and location of the recirculation zone, shown in oil-streak measurements, and to the measured separation efficiency of particles for each OSG.

High-speed video shows how the efficiency of small particles is being adversely affected by these dynamic flow structures, revealing the formation of a wall-normal vortex. This vortex could be the result of reversed scavenge flow and high scavenge flow occurring simultaneously at adjacent span-wise locations. These vortices have been identified as a critical flow structure hampering IPS efficiency for small particles. 
To understand the effect these flow structures have on separation efficiency, MP-PIV measurements for $10 \mu \mathrm{m}$ and $35 \mu \mathrm{m}$ particles were recorded for the three IPS geometries. The particle paths are characterized by steady inflow of particles into the bifurcation zone as a result of the upstream turns. This action allows most of the particles to enter the scavenge leg demonstrating successful particle separation. However, some particles are ingested into the core flow, resulting in inefficient operation. Both the $10 \mu \mathrm{m}$ and $35 \mu \mathrm{m}$ particles also become entrained in the recirculation zone, which gives them a greater chance to be transferred into the core when an ingestion event occurs. Overall, the $35 \mu \mathrm{m}$ particle pathlines show more evidence of inertial motion than the $10 \mu \mathrm{m}$ particles, giving them higher separation efficiency, but both particle sizes are still affected by the unsteady fluid forces in the IPS.

The COR for particles impacting the OSG near the recirculation zone varied with the incoming velocity. Particles are entering at high enough velocities that inelastic particle-wall collisions are occurring. These collisions match with well with experiments and theory. Based on the impact angle, the $35 \mu \mathrm{m}$ particles that impact the wall appear to come straight across the bifurcation region. However, the pathlines of the $10 \mu \mathrm{m}$ particles are not as straightforward, as the smaller particles are influenced by the fluid forces to a much greater extent than the larger ones.

\subsection{Final Conclusions}

This work has revealed some of the mechanisms inherent in the fluid-particle dynamics that lead to inefficiency within an IPS, particularly the instances of reversed flow and the wallnormal vortex in the scavenge leg. This unsteadiness in the scavenge airflow is significantly affecting the particle trajectories, resulting in a loss of separation efficiency, especially for particles with $S t_{D}<10$. 
These conclusions have significant ramifications for the particle separation community. More detailed experiments and simulations of the flow field are required to mitigate the unsteadiness in the scavenge flow. The previous computational work in IPS design has focused significantly on particle bounce and drag models, which appear to be reasonable for the mean flow. However, this study shows that using the mean flow is insufficient to capture the dynamic effects of the fluid on the particles that lead to loss in efficiency.

\subsection{Recommendations}

This work has laid the groundwork for excited new revelations in IPS technology by elucidating the unsteady dynamics in an IPS. Further work is needed to improve computational models of IPS systems, to understand the three-dimensional aspects of the flow, and to determine the correlation of the multiphase interactions.

IPS design can no longer be based on average fluid measurements without considering the effects of dynamic airflow on the particles. Previous computational work was limited to these simple and less extensive averaged calculations. Utilizing advances in computer technology and the computational techniques such as large eddy simulations could further elucidate the dynamics present in throughout the IPS.

Further research is needed to fully understand the three-dimensional, span-wise effects discovered in the PIV and high-speed video. Of particular interest is resolving the span-wise velocity component using 3-D PIV or measuring cross-plane 2-D PIV. However, difficulties may emerge due to the restricted optical access allowed by the IPS test section. 2-D PIV is simple because it only requires one camera perpendicular to the test section, where 3-D PIV requires multiple cameras at various angles to the test section. Unfortunately, due to the 
geometry of the IPS, there is limited optical access to anything but a perpendicular field of view.

Definitive correlation of the fluid and particle velocities will aid in understanding the exact influence of the unstable fluid dynamics on the particles. Dual Phase PIV would contribute to time correlated results for the fluid and particle flow fields. One method of Dual Phase PIV is to utilize pre-processing algorithms to distinguish between small particles and large particles based on the amount of light reflected to the camera. This method works well when there are two disparate sizes of particles used in the measurement (e.g. $1 \mu m$ olive oil and the larger glass spheres). Dual Phase PIV can also be achieved using another method where particles are tagged so they reflect at a different wavelength. These different wavelengths can be distinguished using band-pass filters, and simultaneous data can be taken using two cameras. Further, the time-correlated turbulence data for the flow field and pathlines for individual particles can be measured using High-Speed PIV. Although almost the entire standard PIV setup can be used for High-Speed PIV, it requires a laser with a fast pulse rate, which tends to be prohibitively expensive.

Ultimately, the understanding of IPS multiphase dynamics will lead to better protection of gas turbine engines operating in severe environments. These improvements in design will allow IPS systems to replace the heavier IBF and vortex tube devices that are currently installed in aircraft today. 


\section{References}

[1] W. J. McAnally, "Investigation of feasibility of integral gas turbine engine solid particle inlet separators," in Proceedings of the 10th Annual National Conference on Environmental Effects on Aircraft and Propulsion Systems, 1971.

[2] R. J. Duffy, "Integral engine inlet particle separator volume ii. design guide," General Electric Company, Tech. Rep. AD-A015 064, August 1975.

[3] D. Breitman, E. Dueck, and W. Habashi, "Analysis of a split-flow inertial particle separator by finite elements," Journal of Aircraft, vol. 22, no. 2, pp. 135-140, February 1985.

[4] B. Vittal, D. Tipton, and W. Bennet, "Development of an advanced vanesless inlet particle separator for helicopter engines," Journal of Propulsion, vol. 2, no. 5, pp. 438-444, 1986.

[5] D. Barone, J. Hawkins, E. Loth, and P. Snyder, "Efficiency of an inertial particle separator," in 51st AIAA Aerospace Sciences Meeting, no. AIAA 2013-1080, January 2013.

[6] I. Langmuir and K. Blodgett, A Mathematical Investigation of Water Droplet Trajectories, ser. Collected Works of Irving Langmuir. Pergamon Press, 1946, vol. 10, pp. 348-393. 
[7] A. Hamed, Y. D. Jun, and J. J. Yeuan, "Particle dynamics simulations in inlet separator with an experimentally based bounce model," Journal of Propulsion and Power, vol. 11, no. 2, pp. 230-235, March-April 1995.

[8] D. Barone, E. Loth, and P. Snyder, "A 2-d inertial particle separator research facility," in 28th AIAA Aerodynamic Measurement Technology, Ground Testing, and Flight Testing Conference, no. AIAA 2012-3290, 2012.

[9] P. Stallard, "Helicopter engine protection," Perfusion, vol. 12, no. 4, pp. 263 - 267, 1997.

[10] A. Filippone and N. Bojdo, "Turboshaft engine air particle separation," Progress in Aerospace Sciences, vol. 46, pp. 224-245, 2010.

[11] A. Hamed, W. Tabakoff, and R. Wenglarz, "Erosion and deposition in turbomachinery," Journal of Propulsion and Power, vol. 22, no. 2, pp. 350-360, March-April 2006.

[12] P. Snyder, "Particle separator for a gas turbine engine," US Patent 6,698,180, March 2003.

[13] W. Prinsloo, P. Villiers, and M. Dijken, "Vortex tube separating device," US Patent 4,985,058, January 91.

[14] P. de la Calzada, R. Vázquez, F. Fernández, and M. S. Segundo, "Particle dynamics simulation for aeroengine intake design," Industria de Turbo Propulsores, Tech. Rep. ISABE 99-7280, 1998.

[15] A. Hamed, K. Das, and D. Basu, "Numerical simulations of ice droplet trajectories and collection efficiency on aero-engine rotating machinery," in 43rd AIAA Aerospace Sciences Meeting and Exhibit, no. 2005-1248. AIAA, 2005. 
[16] F. Saeed and A. Z. Al-Garni, "Analysis method for inertial particle separator," Journal of Aircraft, vol. 44, no. 4, pp. 1150-1158, July-August 2007.

[17] M. E. Taslim, A. Khanicheh, and S. Spring, "A numerical study of sand separation applicable to engine inlet particle separator systems," Journal of the American Helicopter Society, vol. 54, no. 4, 2009.

[18] G. O. Musgrove, M. D. Barringer, K. A. Thole, E. Grover, and J. Barker, "Computational design of a louver particle separator for gas turbine engines," in Proceedings of ASME Turbo Expo, no. GT2009-60199. ASME, 2009.

[19] M. E. Taslim and S. Spring, "Numerical study of sand particle distribution, density, and shape effects on the scavenge efficiency of engine inlet particle separator systems," Journal of the American Helicopter Society, vol. 55, no. 2, 2010.

[20] L. Y. Jiang, M. Benner, and J. Bird, "Assessment of scavenge efficiency for a helicopter particle separation system," Journal of the American Helicopter Society, vol. 57, 2012.

[21] A. B. Barta, W. A. Bennet, B. R. Vittal, and M. R. Krishnan, "Design and development of a compact bifurcated turboprop inlet," in 17th Joint Propulsion Conference, no. 91-2017. AIAA/SAE/ASME, June 1991.

[22] J. H. Bell and R. D. Mehta, Contraction Design for Small Low-Speed Wind Tunnels. Stanford, CA: Joint Institute for Aeronautics and Acoustics, 1998.

[23] A. Pope, Low Speed Wind Tunnel Testing. New York: Wiley, 1984.

[24] F. M. White, Fluid Mechanics, 5th ed. New York: McGraw-Hill, 2003.

[25] R. J. Goldstein, Fluid Mechanics Measurements, 2nd ed. Washington D.C.: Taylor \& Francis, 1996. 
[26] J. R. Taylor, An Introduction to Error Analysis, 2nd ed. University Science Books, 1997.

[27] B. Johnson, J. G. Leishman, and A. Sydney, "Investigation of sediment entrainment in brownout using high-speed particle image velocimetry," in 65th Annual National Forum of the American Helicopter Society, Inc, 2009.

[28] L. A. Klujszo, M. Rafaelof, and R. K. Rajamani, "Dust collection performance of a swirl air cleaner," Powder Technology, no. 103, pp. 130-138, 1999.

[29] G. Donsì, L. S. Osséo, and M. Schenato, "Experimental characterization of a short retention time gas-solid," Powder Technology, no. 85, pp. 11-17, 1995.

[30] Z. Ji, Z. Xiong, X. Wu, H. Chen, and H. Wu, "Experimental investigations on a cyclone separator performance at an extremely low particle concentration," Powder Technology, no. 191, pp. 254-259, November 2009.

[31] E. Loth, "Drag of non-spherical solid particles of regular and irregular shape," Powder Technology, vol. 128, pp. 342-353, March 2008.

[32] D. Barone, E. Loth, and P. Snyder, "Fluid dynamics of an inertial particle separator," in 52nd AIAA Aerospace Sciences Meeting, no. 2014-1314. AIAA, January 2014.

[33] K. J. Finstad, E. P. Lozowski, and E. M. Gates, "A computational investigation of water droplet trajectories," Journal of Atmospheric and Oceanic Technology, vol. 5, pp. 160-170, February 1988.

[34] D. Barone, J. Hawkins, E. Loth, and P. Snyder, "Inertial particle separator efficiency using spherical particles," in 49th AIAA/ASME/SAE/ASEE Joint PropulsionConference, no. AIAA 2013-3666, San Jose, CA, July 14-17 2013. 
[35] A. Melling, "Tracer particles and seeding for particlei velocimetry," Meas. Sci. Technol, vol. 8, pp. 1406-1416, October 1997.

[36] C. Reagle, J. Delimont, W. Ng, S. Ekkad, and V. Rajendran, "A novel optical technique for measuring the coefficient of restitution of microparticle impacts in a forced flowfield," in Proceedings of ASME Turbo Expo, Copenhagen, Denmark, June 11-15 2012.

[37] C. Reagle, J. Delimont, W. Ng, and S. Ekkad, "Study of microparticle rebound characteristics under high temperature conditions," in Proceedings of ASME Turbo Expo, San Antonio, TX, June 3-7 2013.

[38] S. Singh and D. Tafti, "Predicting the coefficient of restitution for particle wall collisions in gas turbine components," in Proceedings of the ASME Turbo Expo 2013, San Antonio, TX, June 3-7 2103.

[39] R. L. Jackson, I. Green, and D. B. Marghitu, "Predicting the coefficient of restitution of impacting elastic-perfectly plastic spheres," Nonlinear Dynamics, vol. 60, pp. 217-229, 2010. 